\title{
ENSEMBLE INTERPRETATIONS OF QUANTUM MECHANICS. A MODERN PERSPECTIVE
}

\author{
D. HOME and M.A.B. WHITAKER \\ Department of Pure and Applied Physics, Queen's University of Belfast, Belfast BT7 1NN, \\ Northern Ireland, UK
}

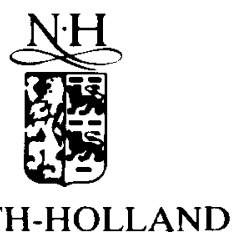




\title{
ENSEMBLE INTERPRETATIONS OF QUANTUM MECHANICS. A MODERN PERSPECTIVE
}

\author{
D. HOME* and M.A.B. WHITAKER \\ Department of Pure and Applied Physics. Queen's University of Belfast. Belfast BT7 INN. Northern Ireland. LiK
}

Editor: 3. Eichler Received August 1991

Contents:

1. Introduction $\quad 225$

2. Interpretations of probability 227

2.1. Interpretations of quantum theory and probability 227

2.2. The notion of probability

2.3. Range of the probability concept

2.4. Objective and subjective probability

2.5. Interpretations that ascribe probability to single cvents

2.6. Relative-frequency ( $\mathrm{rf}$ ) interpretation

2.7. Axiomatic method

2.8. Remarks and conclusions

3. Orthodox interpretations and their problems

3.1. What does "orthodox" mean?

3.2. Von Neumann and the Princeton interpretation

3.3. Bohr, Copenhagen and complementarity

3.4. Summary

4. Ensemble interpretations: a general survey

4.1. Types of ensemble interpretation

4.2. Completeness

4.3. Einstein (Fine, Rosen)

\author{
4.4. Ballentine (Popper) \\ 4.5. Developments of the ensemble interpretation \\ 4.6. Developments in stochastic interpretations \\ 5. Ensemble interpretations: analysis \\ 5.1. PIV interpretations: general remarks \\ 5.2. PIV interpretations and the hidden-variables "im \\ possibility" proofs \\ 5.3. The minimal ensemble \\ 5.4. Measurement and collapse of wave function \\ 5.5. Duane's theory \\ 5.6. General discussion of ensemble interpretations \\ 5.7. Unsharp joint measurements \\ 6. Application to specific problems \\ 6.1. EPR problem \\ 6.2. Schrödinger's cat \\ 6.3. The quantum Zeno (watched-pot) "paradox" \\ 6.4. Bell's theorem \\ 7. Conclusions \\ References
}

269

271

275

$2 x+1$

2801

$2 \times 2$

$2 x+$

280

290

290

245

398

248

$3(1)$

313

3217

310

312

\section{Abstract:}

A discussion of aspects of probability relevant to the differing interpretations of quantum theory is given. followed by an account of so-called orthodox interpretations of quantum theory that stresses their flexibility and subtlety as well as their problems. An account of ensemble interpretations is then presented, with discussion of the approaches of Einstein and Ballentine. and of later developments. including those interpretations usually called "stochastic". A general study of ensemble interpretations follows, including investigation of PIV (premeasurement initial values) and minimal interpretations, an account of recent developments. and an introduction to unsharp measurements. Finally. application is made to particular problems. EPR. Schrödinger's cat, the quantum Zeno "paradox", and Bell's theorem.

\footnotetext{
* Permanent address: Physics Department, Bose Institute, Calcutta 7000009. India.
} 


\section{Introduction}

Ensemble interpretations of quantum theory contend that the wave function describes an ensemble of identically prepared systems. They are thus in contrast to "orthodox" or "Copenhagen" interpretations, in which the wave function provides as complete a description as is possible of an individual system. A study of the relative merits of these two classes of interpretation, and in particular of the extent to which ensemble interpretations may avoid the well-known difficulties of orthodox interpretations, is the main subject of this review.

For purposes of introduction, the definitions have been stated in the boldest of terms, and much of the work of the following sections will be to analyse and clarify the basic ideas. For both orthodox and ensemble cases, we have used the plural - "interpretations" - to emphasise that each class contains several variants, and, particularly on the ensemble side, it will be a major task to distinguish between them. The most important division will be between what we have previously called [1] PIV ensemble interpretations - ensemble interpretations with pre-assigned initial values for dynamical variables, and what Gibbins [2, p. 76] has called minimal ensemble interpretations, which carry no such superstructure.

Distinction between different interpretations, either of the same general class or of different classes, will be made only partly on the basis of abstract definition, which may be rendered free of ambiguity only with the greatest of difficulty. Rather we will make more use of their handling of specific physical situations.

It is convenient to make an immediate comment concerning the relation between ensemble interpretations and hidden-variable theories. The existence of hidden variables would explain why one uses an ensemble interpretation; the ensembles would consist of systems with all possible distributions of values for the hidden variables. However use of an ensemble interpretation does not imply acceptance of hidden variables, to the possibility of which it remains neutral [3, p. 374]. (An opposite tentative opinion may also be found [4, p. 447].)

Ensemble interpretations of quantum theory have practically as long a pedigree as orthodox interpretations. If Bohr [5] and Heisenberg [6] may be regarded as the founders of the latter, Einstein [7] was an early supporter of the view, which he maintained until the end of his life, that quantum theory represented a correct and complete statistical theory of ensembles, but not a theory of elementary processes. In other words he regarded the theory as incomplete, which was indeed the conclusion of the famous Einstein-Podolsky-Rosen (EPR) argument [8]. (For a fuller account, see section 4.3.) In the early days of the quantum theory, ensemble interpretations had other strong advocates - Slater [9], Kemble [10], Popper [11], Langevin [12], and Margenau [13], but the very great influence of Bohr meant that, at the very least till the 1950s, such views remained peripheral. One should also mention the famous argument of von Neumann [14], which claimed to show that hidden-variable theories were impossible; in view of our remarks immediately above, it would be expected that acceptance of such arguments would at least dampen enthusiasm for ensemble interpretations.

One exception to this situation was in Soviet Russia, where the controversy was complicated by political considerations [4]. It was felt by some that Bohr's views on quantum theory, in particular his espousal of complementarity, amounted to idealism, and hence were opposed to dialectical materialism. This view was enforced with some severity for a decade or so after the Second World War, and, in keeping with it, ensemble interpretations were preferred to that of Bohr. Such a view was propounded by Blokhintsev in particular [15]. Fock [16] did remain able to reject these views, however, and 
stimulated a more favourable assessment of Bohr's philosophy from the late 1950s, though Blokhintsev himself continued to support an ensemble interpretation [17].

It was in the 1950s that the previously solid support for Bohr in the West began, exceedingly gradually, to be eroded. The Einstein volume [18] in the Library of Living Philosophers had just been published; this included Einstein's own clear exposition of his position, and his explanation of his differences with Bohr. In 1952 Bohm [19] produced an explicit hidden-variables theory in defiance of vón Neumann. In the following decade. Bell, with the utmost clarity, showed exactly what was wrong with von Neumann's argument, and others to the same conclusion [20]. but, building on the EPR argument, he also demonstrated that no local hidden-variables theory could agree with quantum mechanics [21].

During this period, the problem of measurement in quantum theory gained in prominence, in particular the question of wave-function collapse at a measurement, postulated by von Neumann [14]. but anathema to many. Another controversial topic in this area was the explicit presence of macroscopic variables in a fundamental theory; orthodox measurement theory required a "cut" between microscopic and macroscopic, but could not position it with any precision [22, p. 188]. One should also mention the Uncertainty Principle: this was indisputably a central component of quantum theory, yet many felt that its meaning remained obscure.

During the $1950 \mathrm{~s}$ and $60 \mathrm{~s}$, a number of alternative interpretations of quantum theory were developed, at least partly in response to these difficulties. The relative-states interpretation was proposed by Everett [23] in 1957. though it was only brought into prominence much later by de Witt [24], who re-titled it the many-worlds interpretation. At about the same time Hartle [25] and Cooper and van Vechten [26] also suggested similar ideas. Following on from pre-war work by Schrödinger [27] and Furth [28], stochastic interpretations were developed by Bopp [29] in particular.

Ensemble interpretations also continued to be proposed in these decades, by Pearle [30| and Park [31] in particular, but it was the article by Ballentine [3] which was most influential. This work. which was published in 1970 in the Reviews of Modern Physics only after an apparently inordinate amount of soul-searching by editor and referees [32], constituted a comprehensive onslaught on the Copenhagen interpretation. Virtually all the problems mentioned above were discussed, and Ballentine claimed to show that the ensemble interpretation (or statistical interpretation, as he called it) was capable of dealing with all of them.

Another important event was the publication, four years after Ballentine's paper, of Jammer's The Philosophy of Quantum Mechanics [4]. This book provided a thorough survey of the highly dispersed literature on the fundamentals of quantum theory, and is still an indispensible source for all those working in the area. Its chapter on statistical interpretations provides an extensive coverage of the work of all those authors mentioned in this introduction, towards the writing of which it has contributed a great deal.

In the years since 1970, the study of the philosophical basis and interpretations of quantum theory has expanded enormously. Those involved have analysed the issues. problems and proposed resolutions of problems mentioned earlier in this introduction, as well as studying newer ideas such as the proposed quantum Zeno effect [33]. To philosophical and theoretical discourse has been added the challenge of experiment. Experiments to test Bell's arguments have been performed, notably by Aspect [34]; Leggett [35] has pushed towards macroscopic quantum effects in such devices as SQUIDs; and the neutron-interferometry experiments of Rauch [36] have made many of the gedanken experiments of the 1920s into real experiments.

During this period. Ballentine's paper has proved to be one of the more stimulating sources of discussion and dialogue, and the role of the ensemble interpretation in withstanding such potential 
difficulties as EPR and the Bell arguments has been the focus of considerable attention. The review article itself has been cited more than 150 times. A typical response may be that of Pippard [37], who does not necessarily underwrite every argument, but nevertheless reports that Ballentine's approach is closer to his own than anything else he has come across (and, incidentally, suggests that the ensemble interpretation is neglected in the substantial collection of papers on quantum fundamentals edited by Wheeler and Zurek [38]). Ballentine himself [39] has recently succinctly restated his own position.

The task of this review is to obtain a modern perspective on ensemble interpretations by concentrating on the literature of the last twenty years. Noncontroversial material presented by Ballentine and Jammer is not re-examined, but arguments presented since the appearance of their works are analysed, and we try to present enough general discussion and argument to ensure that readers may reach their own conclusions on the degree of success achieved by the class of interpretations.

An author given much attention by Jammer [4, pp. 453-465], but whose work will not be discussed here is Landé, universally known for his pioneering work on atomic spectroscopy, and in 1951 an author of a text supporting the Copenhagen interpretation [40], but soon afterwards a staunch opponent [41]. Landé was totally repelled by wave-particle dualism, and produced a version of the ensemble interpretation that was unitary in that it invoked only particle-like properties. Diffraction, a difficult point for corpuscular theories, was handled by the "third quantisation rule" of Duane [42], which says that when a particle interacts with a crystal of periodic length $l$, the momentum of the particle may change by multiples of $h / l$.

Landé's aim was to develop the results of quantum theory from the smallest possible number of fundamental principles. He laid great stress on the connection between thermodynamics and quantum theory, his entry to the former being via his solution of the Gibbs paradox. His work took him well beyond the confines of the conflict between orthodox and ensemble interpretation. Indeed Jammer [4, p. 461] quotes the comment of Mehlberg [43] that Landé refrained from discussing this particular point. Thus his work is not centrally a study of the area under review here.

The plan of the review is as follows. Section 2 sketches an account of the various interpretations of the concept of probability. The particular aim here is to distinguish between those interpretations which consider that the concept may be discussed for a single system, and the frequency interpretation, which applies it only to an ensemble. There is a strong logical connection, and perhaps an even stronger psychological one, between this conflict and the one between orthodox and ensemble interpretations of quantum theory.

Section 3 discusses orthodox interpretations, and the various problems that beset them. Section 4 then introduces ensemble interpretations, emphasising differences between the various members of the group as much as between this group as a whole and that of orthodox interpretations. In section 5 , a critical survey is carried out of the merits of ensemble interpretations. While this section contains general arguments concerning quantum measurement, section 6 considers application of ensemble interpretations to specific problem areas - EPR, Schrödinger's cat (and the "cut" between microscopic and macroscopic), quantum Zeno, and Bell's theorem and locality. Section 7 presents concluding comments.

\section{Interpretations of probability}

\subsection{Interpretations of quantum theory and probability}

The fundamental difficulty in interpreting quantum theory is that it provides in general only probabilities of obtaining given results. Thus much of any discussion of quantum theory must depend on 
what one means by probability - how one defines or interprets the term. The distinction between those interpretations of probability that assign a value of probability to an individual event, and the relative-frequency ( $\mathrm{rf}$ ) definition, which uses the term only for a sequence of trials, may appear to parallel that between orthodox and ensemble interpretations of quantum theory.

Indeed, in discussion at a conference of 1968 , Post [44, p. 210] conjectured, "the only real difference between the different views expressed [on quantum interpretation] ultimately boils down to a difference in the interpretation of probability". More generally, Jammer [4, p. 7] suggests, "the different interpretations of probability in quantum mechanics may even be taken as a kind of criterion for the classification of the various interpretations of quantum mechanics".

Thus it is decidedly relevant to commence this review by analysing the various interpretations of probability. Our approach will not be a narrow one, related directly to the specific area; it would be a fatal error to choose an approach to probability which suits our views on quantum theory, and then to use the former to justify the latter! Useful wide-ranging works are those of Weatherford [45] and Lucas [46]. We commence with a few general remarks (sections 2.2 to 2.4).

\subsection{The notion of probability}

The notion of probability is a fundamental one. One may conceive of primitive man, after crystallising a few of the basic physical features of life, such as food, shelter and so on, being struck by the uncertainties and risks of life - the occurrence of severe weather, the lack of food, the presence of predators - and developing the ideas and language of probability. What is absolutely clear is that such ideas could not be expressed using the previous vocabulary of concrete terms; since this was the only available vocabulary, they had to be left undefined. We should be neither surprised nor alarmed by this; Jeffreys [47, p. 407] demonstrates by his "infinite regress argument" that one can never define all the concepts of a system.

Thus Lucas [46, p.1] notes, "we have great difficulty in saying what probabilities are"; Reichenbach [48, p. 3], "although we apply the concept of probability in daily life as a matter of course, we find it difficult to say what we mean by the concept "probable",. Poincare $[49 ; 50$, p. 99] says that the calculus of probability rests on "an obscure instinct we cannot do without", but also [51, p. 24; 52, p. 67] that "it is hardly possible to give any satisfactory definition of probability". Lewis says that he could not explain probability to anyone who did not already possess a primordial sense of probability [53, p. 320; 45, p. 3].

There are, of course, circumlocutions available, such as "defining" probability as degree of confidence or belief or rational belief ("definitions" which, whatever else they achieve, stir up the hornet's nest of subjectivity or objectivity - see section 2.4). Keynes, for example [54, p. 8] says that a definition of probability is not possible "unless it contents us to define degrees of the probability relation by reference to degrees of rational belief". Von Mises [52, p. 3] shows that dictionary definitions provide only substitutions of one word for another, and is likewise unimpressed [52, p. 136] by the legal "definition" of games of chance as "games of aleatoric character".

Jeffreys [47, p. 14] uses the phrase "degree of confidence" but does not appear to regard this as a definition of probability. Indeed he is prepared to be on the offensive concerning lack of such a definition. "Part of the objection to probability as a primitive notion", he writes [47, p. 379], "is connected with the belief that everything is vague until it is defined in words. Such a belief omits to recognise that some things are perfectly intelligible before any definition is available. To try to define such things can result only in defining them in terms of something less immediately intelligible." He 
goes on to say [47, p. 380], "Definitions add clarity when something new is defined in terms of something already understood; but to define anything already recognizable is merely to throw valuable information into the wastepaper basket." Jeffreys quotes W.E. Johnson's remark that some things are "so generally and universally understood that it would be mere intellectual dishonesty to ask for a definition".

Von Mises [52, p. 3] aims at a "synthetic" definition in which a way of using the term is established, as opposed to an "analytic" definition. This concurs with Jeffreys [47, p. 380] who says, "all that can be done is to point to instances where the phenomenon in question arises". However an immediate and crucial difference appears when one asks the question: to what extent is common usage felt to establish an effective synthetic definition. Von Mises asserts that this is not the case; "The value of a concept is not gauged by its correspondence with some usual group of notions." He makes [52, p. 5] the analogy with the term "work", used colloquially in a variety of ways, but defined scientifically in a highly specific and refined sense. Similarly he suggests that conventional uses of the word "probability" may be ignored in the development of a scientific usage.

Jeffreys [47, p. 393] disagrees. He believes, "the real reason for the difficulty in the understanding of the theory of probability is . . that the fundamental ideas and general language has overlooked them, and when they are stated it is immediately taken for granted that they must mean something too complicated for ordinary language, and a search is made for something to satisfy this condition. The truth is that they are too simple for ordinary language."

This is the source of the major divide in the foundations of probability. Jeffreys' view is that the colloquial use of the word "probability" is not vague or indiscriminate, but highly specific. Each individual has a clear idea of what he or she means by the term, and this idea is (at least by and large) shared with others. The difficulty felt with the concept is not that it is vague, or has different meanings from individual to individual, but merely that, as already discussed, its analytic definition is not possible. Those who support this view (either completely, or to a considerable extent) will have no qualms in producing interpretations of probability which allocate a value of probability to an individual event the throw of a dice, for example. Such interpretations - the classical interpretation of Laplace, the logical relation interpretation of Keynes, Jeffreys and Carnap, the subjective interpretation of de Finetti and the propensity interpretation of Popper - are discussed in section 2.5 .

(In saying that such interpretations accept common usage in this way, it is not necessarily implied that they do so in all respects. Interpretations of probability usually make a virtue of corresponding to primitive ideas for as long as they actually do so. For example, de Finetti [50, p. 111], supporting the subjective interpretation, claims that it is "without doubt the closest to that of 'the man in the street'; better yet, it is that which he applies every day in his practical judgements. Why", de Finetti asks, "should science repudiate it? What more adequate meaning could be discussed for the notion?"; but he feels impelled to add in a footnote, "probability theory is not an attempt to describe actual behaviour".)

The chief opponent of these interpretations is the relative-frequency (rf) interpretation of von Mises [52] and Reichenbach [48]. This too starts off as a "simple common-sensical theory based on the actual practice of ordinary men" (in the words of Lucas [46, p. 96]). It rejects the idea of a probability for a single event, but fastens on the well-known, though, as will emerge, in fact rather subtle connection between probability of occurrence for a single event (if the concept is accepted) and relative frequency of an occurrence in a long sequence of events. Clearly the idea is attractive as it appears possible to give a straightforward empirical definition of what is meant by probability. The extent to which the interpretation retains its simplicity and achieves its aims is discussed in section 2.6.

In section 2.7 a third general approach to the foundations of probability, due to Kolmogorov [55] is discussed very briefly. 


\subsection{Range of the probability concept}

Before commencing discussion of the individual interpretations of probability, we wish to make two more introductory remarks. The first concerns the range of areas where we would be willing to use the term "probability". In common parlance we might be prepared to discuss the probability that

(1) an electron has $S_{z}=1 / 2$ (or that a measurement of $S$ will give the value $1 / 2$ );

(2) an alpha particle will be scattered by a given angle (or within a given range of angles);

(3) a tossed coin will come down heads;

(4) a measurement of a quantity known to have a value exactly 2 will produce a value between 2.11 and 2.12 ;

(5) an experimental measurement of 2.11 confirms that the quantity measured has a value 2:

(6) the theory of Special Relativity is true;

(7) life exists in the universe apart from on earth;

(8) a football match will be won by side A:

(9) it will rain tomorrow;

(10) the battle of Marathon was fought in 490 B.C. [46];

(11) a specific individual (name selected from telephone directory) has a blue right eye [47]:

(12) a specific individual (selected as above) will die in the coming year;

(13) a specified marriage will end in divorce:

(14) what one "partially remembers", for example that a train to Paris is scheduled to leave at 10.15 , is actually true:

(15) that the figure at the billionth decimal place of $e$ is 5 ;

In many cases [for example (1), (4) and (8)] one would require additional information; in others one would only contribute if one had specialised knowledge [for example (6), (7) and (10)]. But it is certain that, for each of the questions, some people could be found to express a relevant probability, either as a number, exact or approximate, or as a more general term, such as highly probable, or very improbable.

Yet the types of ideas expressed are so diverse that it would seem almost impossible to produce a theory able to discuss all the examples on an equal footing. Formally it may be advantageous [54] to replace "probability of an event" [in (1), for example] by "probability of a proposition", the proposition being that a measurement of $S_{z}$ gives $1 / 2$, that Special Relativity is true, and so on. This produces a formal unity, but one may question whether it does any more than this, or whether it merely identifies verbally concepts that are fundamentally disjoint.

From our perspective the general question is of interest, because some interpretations handle readily far more items on the list than others. In particular, the rf interpretation can handle easily only (1), (2) and (3), and, with suitable data available (11), (12) and (conceivably) (13).

Of course the first two of these cover the kinds of example used in scientific work (with the exception of statistical analysis of, and inductive reasoning from, experimental results where (4) and (5) are required). (From our list we can understand Weatherford's point $[45, \mathrm{p}$. 74] that if interpretations are used on the whole by scientists and actuaries.) So, for the purposes of this paper, it would seem quite possible to disregard the other items on the list, regarding them as "unscientific", and use the rf. As explained at the end of section 2.1, we would regard this course of action as shallow.

For comparison with the if interpretation, the classical interpretation can only readily tackle (3) (and then only if the coin is fair); the propensity interpretation may discuss the same items as the rf, and also, with rather less strain than the latter, such items as (8), (9) and (13). The logical relation and subjective interpretations may discuss all the items on the list (though see Jeffreys [47, p. 38] for a 
comment on the last item). (Weatherford adds to his previous remark that card players use the classical interpretation, logicians the logical interpretation, and psychologists the subjective interpretation.)

\subsection{Objective and subjective probability}

The other introductory comment is on the uses of the terms "objective" and "subjective" as applied to probability. These terms are often used as though their meaning is obvious; in fact, from different points of view, very different meanings appear natural, and the confusion bedevils much discussion of the applications of probability (for example ref. [44, pp. 202-219]). Much of the problem is that the word "subjective" is usually used in a perjorative fashion, but without sufficient analysis. (Sometimes the word is used neutrally, as by Margenau [44, p. 211], or with approval, in particular of course, by the subjectivists themselves (section 2.5.3).)

Let us first ignore the complications introduced by quantum mechanics and its accompanying indeterminism (and certainly even the most up-to-date and "scientific" approach to probability must be able to handle the classical case). There are then at least two distinct meanings that may be attached to the subjective/objective distinction. Perhaps the most natural is to treat "objective" as meaning "consensible", that where all may (and, in some sense, should) agree on the value of the probability. Thus (1), (2) and (3) on the list in the preceding section, are examples of objective probability on this definition, but (8), (9), (10) and (13) are examples where there are (virtually) bound to be differences of opinion, and thus the probabilities will be termed subjective. The distinction will be useful, though perhaps not hard-and-fast. As the studies of meteorology, ancient history and psychology/sociology progress, their exponents must object to their judgements on such matters as (8), (10) and (13) being termed "subjective" - to the extent that this term is felt to denote little more than irrational prejudice. There will certainly be some states of the weather when an opinion on (8) may be felt to be as objective as those on (1), (2) or (3).

A very different distinction that may be made between "objective" and "subjective" is that which makes an "objective" probability a "real" probability, as opposed to merely an uncertainty on the part of a particular observer resulting from personal ignorance of the full facts. On the list, (3), at least at first sight, will seem to be clearly objective because nobody knows (or could know) on which side the coin will fall. So will, for example, (8) and (9). But (14) is definitely subjective - the fact is not genuinely doubtful, and it is just personal ignorance that requires the individual observer to assign a probability.

To give a more concrete example (based on one of Weatherford [45, p. 45]), consider two boxes, one with two white balls and one black, the other with two black balls and one white. The observer selects a box, shakes it, then selects a ball, and the problem is to calculate the probability of choosing white or black. The trivial calculation may be done in two stages: probability of selecting a particular box, and then, for each box, probability of selecting a ball of a particular colour. According to the definitions being studied at the moment, the first probability would be called subjective, or not real. The observer does not know which box contains two white balls, but that is just individual and avoidable ignorance; others may know, and the observer could always look. The second probability, however, is objective, since once one has shaken the box, nobody could know which of the three balls it contains would be chosen.

Such is an apparently reasonable position, but it is one fraught with difficulty. For (remembering we are at the moment in a deterministic world) such events as tossing the coin are in principle predetermined. The Laplace demon would know what result would be obtained in the coin-tossing for 
example, and in that sense, all probabilities become "subjective", which renders our classification useless.

We may attempt to save the classification by deciding that objective probabilities are those which are "predicated on the best knowledge normally available to human beings" [45, p. 45]. This will restore our analysis of the two-box problem above. But even this procedure may be questioned. Reichenbach [48, p. 150] points out that, at a roulette wheel, the croupier calls out "rien ne va plus" at (or, presumably, rather before) the time when the wheel has slowed down sufficiently that a gambler with smart eyes and judgement might be able to work out where the ball will end up. At this moment, according to our most recent criterion, the probability will change from objective to subjective. This shows that again the classification may be useful in many cases, but is certainly not hard-and-fast.

A not totally dissimilar comment may be made concerning example (11). If we pick the name from the UK telephone directory, it might be felt that the probability of finding an individual with a blue right eye might be the average for a UK citizen. But such is not the best information normally available to human beings. From the name, conceivably from the address, we could deduce a possibility or even a near certainty on grounds of national background that the probability would be different. We could again conceivably know relatives of the individual, and the colour of their eyes, and that knowledge again could change considerably the value of the probability (or the value to be attributed to the probability; note that by this stage of the argument we may feel happier with this more apparently subjective formulation). Knowledge of the colour of the left eye could have an even more dramatic effect. Lastly, in fact, of course, the individual either has a blue right eye, or does not; in other words the fully "objective" probability becomes either one or zero. To put it another way, the probability concept is unnecessary from a strictly objective point of view; to construct a theory on the basis of probability is to attempt to "generate knowledge from ignorance" [45, p. 47; 56, p. 167].

Clearly there is a great conceptual problem. However keenly an interpretation may "hunt down the individual" in an effort to be totally objective, in the end it must "back off" to accepting a notional value for some collective, because, in the individual case, the probability disappears. Each interpretation must cope in its own way. Lucas [46, p. 51] defines a probability for a range or universe of discourse; probability judgements, he says, are always covertly universalisable. Logical relation and $\mathrm{rf}$ interpretations reach their own solutions (sections 2.5.2 and 2.6). (Elsewhere, the propensity interpretation, as often, follows the rf; the subjective interpretation glories in the lack of objectivity; the classical interpretation is really too narrow in application to be much troubled.) The only comment that is necessary here is that, in "backing off", the logical-relation interpretation is often accused, by supporters of the $\mathrm{rf}$ interpretation and others, of retreating to subjectivity (using the term perjoratively), and it may be lumped in with the avowedly subjective interpretation of section 2.5.3. This cannot be just; as we have seen, logical-relation and rf interpretations have the same problem, and the steps they take to avoid it are analogous. (See, for example, the discussion of Weatherford [45, p. 210].)

As stated at the outset, the discussion so far has been deterministic, and so pre-quantum. When quantum ideas are introduced, things are different. Even when all subjective elements (on our most recent definition) are removed, there does remain a "real" objective probability - as in (1) on our list. (Hidden variables, which could remove determinism, and so get rid of "objective" probability are not considered here.)

This leads to the following criterion between objective and subjective probabilities in quantum theory. A pure state vector (idempotent density matrix) corresponds to an objective probability. When the state vector is not pure (non-idempotent density matrix), there is a subjective probability, often in 
addition to the objective one. It must be said, though, that while this use of terms follows naturally from the previous discussion, and is self-consistent, it does not connect particularly clearly with other usages of the terms "objective" and "subjective". Also, if one accepts it, it must follow that all probabilities in classical physics are subjective.

These ideas seem to correspond with some of Margenau's [44, p. 212], though it must also be said that other comments of Margenau in the same discussion [44, pp. 211, 219] do not appear to be totally consistent with this comment.

\subsection{Interpretations that ascribe probability to single events}

\subsubsection{Classical interpretation}

The classical interpretation of probability was developed by the founders of the probability calculus such as Laplace and Jacob Bernouilli. Laplace wrote his Philosophical Essay on Probabilities [57] in 1819 , though the full articulation of the classical position really had to wait Todhunter's retrospective account of 1865 [58].

The classical interpretation is especially directed towards an account of gambling. It relies on finding a number of equally possible cases, and measuring the probability as "a fraction whose numerator is the number of favourable cases and whose denominator is the number of all the cases possible" (Laplace $[57$, p. 6]). (The status of this "definition" is discussed later in this section.) Laplace defines "equally possible cases" as "such as we may be equally undecided about in regard to their existence" [57, p. 6]. Bernoulli [45, p. 29] developed a rule that "alternatives are always to be judged as equiprobable if we have no reason to expect or prefer one over another", a rule which Keynes [54, p. 41] called the Principle of Indifference.

The classical interpretation could deal highly successfully with matters within its brief, for example, the tossing of fair dice. Equally obviously, as soon as one left this extremely limited area of equiprobable alternatives, for example turning to biased dice, it could not contribute. Indeed, with the formal definition above, it could not be applied to actuarial cases, births, deaths and so on. But, as Weatherford [45, p. 31] points out, such workers as Laplace [57, p. 140] were highly involved in investigating mortality tables, and the incidence of smallpox in particular, and would also consider biased dice [57, p. 57].

Von Mises [52, p. 70; 45, p. 59] claims that, whenever they wanted to, the classical theorists forgot their own definition, and replaced it by a frequency-based one. Weatherford [45, p. 27] comments that, at these moments, their "official" definition failed them "and they tended to abandon it, but they did not replace the official definition with another in terms of likelihood, relative frequency, etc.". "Instead", Weatherford goes on, "they just continued to use the word 'probability' as if everyone understood its meaning, while actually employing methods and concepts which are clearly inconsistent with what they said 'probability' meant."

In fact, as Weatherford [45, p. 70] also suggests, the clearest appreciation of the situation may be obtained by thinking of Laplace's famous "definition" merely as a way of calculating probabilities in the equiprobable case. Very much in agreement with our comments on section 2.2, Laplace did not try to define probability, because "everyone knew what it meant already, and theorists of the time did not bother to define words in common usage". "We have a notion of probability (whether linguistic or intuitive)", Weatherford suggests [45, p. 244], "but we cannot hope to break out of the circle of synonyms to explain that concept because it does not depend on anything else for its force and validity". 
[The remaining problem for the classical interpretation is that what "everyone knew" convoluted two concepts, probability (of an individual event) and relative frequency, which are intimately connected but not the same; this problem is discussed in section 2.6.]

\subsubsection{Logical-relation interpretation}

The logical relation (or a priori) interpretation is particularly associated with the names of Keynes [54] and Jeffreys [47]. One should also mention Carnap [59], who acknowledges both relative-frequency and logical-relation interpretations, and relates them to different types of probability, probability, and probability ${ }_{1}$ respectively. though his main achievements have been to the logical-relation interpretation.

In this interpretation (or group of interpretations), the probability is known or established or at least exists a priori, in obvious contrast to the rf interpretation, where it is empirical and must be measured. Probability is a relationship between two statements, for example the statement " $X$ is fifty years old" (which may be called the hypothesis or evidence or data, and is denoted by $h$ here) and the statement (called the proposition, $a$ ) " $X$ will die in the next year". A notation such as $a / h$ (Keynes) or $P(a \mid h)$ (Jeffreys) may be used for "the probability of proposition $a$ on data $h$ ".

In many ways the chief area of contention has been the presence of the data, $h$, in the relation. The reason has been foreshadowed in section 2.4 , where it has been shown that, if one merely asked for the probability that (named) $X$ will die next year, classically it must be 1 or 0 . The form of probability above enables us to consider all individuals who are 50 years old, suppressing all further information about $X$.

For Jeffreys and Keynes as well, presence of the data is crucial. Jeffreys [47, p. 15] writes, "Omission to recognise that a probability is a function of two arguments, both propositions, is responsible for a large number of serious mistakes". and Keynes [54, p. 40] writes in similar fashion.

For opponents, supporters of the rf interpretation and others, it is a grave drawback, leading to subjectivity (since probability appears to be judged by each individual, according to that person's information). The probability of the truth of a proposition, they may say, should be a definite quantity, not relative to, or dependent on, anything; rf supporters will say that this value may be found objectively from a sequence of trials. Toulmin [60, p. 80] says, "Many of us will never agree that probability is relative to evidence in any more than an epigrammatic sense", and Ayer $[61 ; 62$, pp. 12. 29] calls insistently for $P(a)$ not $P(a \mid h)$. These authors seem to ignore the point made in section 2.4 that, classically, $P(a)$ is 1 or 0 .

This area of discussion has rather drawn attention away from a question which, from our point of view, appears rather more important: how is the value of $P$ obtained? In section 2.2 we saw that both Keynes and Jeffreys were rather negative on the possibility of finding an adequate definition for probability. To obtain values, Keynes often [54, ch. 4] reverts to the Principle of Indifference of the classical interpretation, though he applies much effort to eliminating its major obvious defects. Weatherford [45, p. 94] points out that, in addition, Keynes does not deny power to frequency arguments. "It is undoubtedly the truth", Keynes says [54, p. 108], "that many valuable judgements in probability are partly based on a knowledge of statistical frequencies", but he would never identify a relative frequency with a probability. In addition, it is a well-known argument of Keynes [54, p. 34] that not all probabilities may be expressed numerically. This idea was contradicted by Jeffreys, who says that Keynes later withdrew it $[47$, p. v].

Jeffreys himself $[47$, p. 15$]$ does start from the idea of probability as degree of confidence that we may reasonably have in a proposition. Without the italicised word, the definition seems purely 
subjective (in the sense of being personal). With it, one is left to wonder what the evidence is that makes the confidence reasonable, and why we do not concentrate on that evidence rather than the confidence it evokes. In practice, Jeffreys uses much the same criteria as Keynes. Neither can produce an adequate definition of probability or a general method of calculating probability values.

Carnap's position [59;45, pp. 85-92] is entirely different. It is based on the abstract principles, and highly technical analysis, of symbolic logic. Carnap uses a number of "simple languages", and the value of probability is obtained as a measure of the inclusion of the range of one sentence in that of another in the particular language. The study of even the simplest cases is very elaborate, and involves the lengthy process of counting individual cases or "predicates".

As an intellectual achievement, Carnap's work is of great importance. It provides a definition of the term "probability" and a formal way of evaluating its value for particular cases. However, as Weatherford [45, p. 143] admits, the success is "more potential than actual. It cannot be said that many practising statisticians or handicappers compute probabilities by counting predicates in a language."

Therefore, in summarising the central idea and achievement of this group of interpretations, we shall leave aside the work of Carnap (and also Keynes' suggestion that a probability need not have a numerical value). We shall say then that a logical probability relation does exist, that it may be expressed as a number, that different methods of calculation are available for some cases, but no general definition or method of calculation is available. (Once one has accepted this idea, the interpretation is highly successful in analysing the applications in many areas.) In a general sense, the conclusions are practically the same as those of the classical interpretation. The techniques and philosophical sophistication have improved immeasurably, but not the core conclusions.

\subsubsection{Subjective interpretation}

We have seen that the logical-relation interpretation lays itself open to the charge of being subjective. In the first place it is not empirical. Secondly, it states explicitly that probability depends on the data available to the individual making the judgement, and is thus personal in that sense. Thirdly, Jeffreys seems at least close to identifying probability with degree of confidence, which again seems to make it a personal concept. It should be said that Jeffreys [47, p. 376] strongly denies this charge of subjectivity.

On the other hand, the advocates of the subjective interpretation glory in such charges. This interpretation gives up altogether any search for a definition of probability, or even the idea that there exists, in any particular case, an "unknown probability" towards which one should be groping. It insists that all probability can be is individual degree of belief, not rational degree of belief. The main proponent of these views, and developer of the substantial body of theory underpinning them, has been de Finetti [50, p. 93; 63], together with original concepts of Ramsey [50, p. 61] and support from Savage [64].

Degree of belief must be measured objectively, and, following centuries of tradition, subjectivists do this via gambling. If one is prepared to bet at evens in a contest between two individuals, in doing so one declares that one rates the probability of either winning as $1 / 2$. (And what one rates it as is the probability.) Acceptance of odds of 3 to 1 against an individual corresponds to announcing a probability of $1 / 4$ for that result, and so on.

While the procedure is admirably clear, there must be suspicion concerning the implied direct relationship between betting behaviour and degree of belief. Indeed Laplace as well as all the main subjectivists have discussed this problem. For a man with 1000 dollars, a gain of 1000 dollars would be quite pleasant, while a loss of the same sum would be a disaster. A rich man may willingly risk the loss 
of a substantial sum; a poor one cannot. Some may refuse to bet at all, or bet only when the odds seem exceptionally good, on moral or other grounds.

Laplace [57, p. 23] discusses moral advantage, which he defines as actual advantage as proportion of personal worth; Ramsey [50, p. 74] discusses the utility of the hypothetical gain; de Finetti [50, p. 102] restricts himself to small bets. But the basic difficulty is not removed; probability theory appears to have become entangled at the most fundamental level in the psychology and economics of gambling.

It has been stressed that, in the subjectivist account, there is no "unknown probability". If you believe that the probability of a coin coming down heads is 0.1 , that is just a lengthy way of saying that, for you, the probability is 0.1 . You may choose later, on the evidence of a number of throws, after thought or advice, or for any other reason, to amend your guess, but, if you do so, you are amending the probability; it would be totally wrong to think of your guess approaching a correct, though unknown probability [50, p. 119].

There are, however, some limits on what guesses may be made for the various possible results of a single event. In a contest between two people, it would be foolish to offer to accept bets at 3 to 1 on, for each contestant, because a set of bets could easily be made against you that would guarantee that you would make a loss, whatever the result. De Finetti $[50$, p. 103] calls making such an offer incoherent behaviour; there is a possibility of a "Dutch book" being made against one. Where one does not lay oneself open to the possibility of a Dutch book, one's behaviour is described as being coherent. Intriguingly, de Finetti is able to show that the demand for coherence in a set of degrees of belief implies the whole of the usual calculus of probability (rules that are usually stated as axioms).

Another important concept of the subjective interpretation is exchangeability [50, pp. 12, 18]. Conventional interpretations of probability talk of sequences of independent identical events, the probabilities relating to which must be the same. The subjective interpretation denies this: each event must be considered on its own merits. In a series of coin-tossings, the probability that the $(n+1)$ th tossing will give heads, that is, the degree of belief on the part of the individual that it will be heads, will probably be different from that for the $n$th tossing, since it will reflect the results of the first $n$ (rather than $n-1$ ) tosses.

At first sight this would seem a recipe for chaos. Indeed Kyburg and Smokler [50, p. 12] comment that until 1931 "the subjectivistic theory of probability remained pretty much of a philosophical curiosity. None of those for whom probability theory was a means of livelihood or knowledge paid much attention to it." Then in 1931 de Finetti introduced the idea of exchangeability. The key point is that, although the probability of the $(n+1)$ th toss does depend on the proportion of heads in the first $n$ tosses, it does not depend on the positions of the heads in the sequence.

With this principle, the subjectivist is able to learn from experience, and after a sufficient number of tosses, may come to assign a probability to the event, equal to the observed relative frequency, practically irrespective of the initial subjective assignment of probability.

With exchangeability, then, when interpreting the results of a sequence of observations, the subjectivist may behave in a broadly similar fashion to someone who believes that there is a definite, though unknown, probability, but from an entirely different conceptual background. This fact enabled the subjective interpretation to take its place alongside other interpretations as a serious attempt to understand probability. Indeed, exchangeability is technically an interesting branch of probability theory even for those who do not have subjective views.

It is necessary to stress one qualification of the above. We have said that subjectivists may behave in the way we have described. For instance, when 100 tosses of a coin have produced nearly 50 heads, 50 
tails, they may be expected to act on the assumption that each probability is near $1 / 2$. But [45, p. 230], we cannot insist that they do so. They may decide the probability of heads is $3 / 4$. In the first place it is certainly possible that this value of probability could give a Bernoulli sequence with equal numbers of heads and tails. (See section 2.6 in particular.) Indeed there is really in any case no necessary reason to connect past experience with future expectation. In the end there is no unknown probability, no rational degree of belief, just degree of belief; if our observer believes he is correct to dismiss the argument about exchangeability, he is entitled to do so.

\subsubsection{Propensity interpretation}

The propensity interpretation is due to Popper $[62$, p. $65 ; 65$, p. 7$]$; it was subsequently elaborated by Mellor [66]. Unlike all the other interpretations of probability discussed here, it was designed specifically to solve the problems of quantum physics. Popper claims that the usual interpretation of probability oscillates between two extremes, which he calls the objective and statistical, and the subjective, based on the idea of incomplete knowledge. The latter, he says, is responsible for the appearance of the observer in the conventional interpretation of quantum theory. As against this, Popper says that his propensity interpretation is purely objective and avoids the appearance of the observer. The interpretation, he claims, "takes the mystery out of quantum theory while leaving probability and indeterminism in it".

After that build-up, the quantum physicist is liable to feel a little let down by the actual content of the propensity interpretation. It is helpful to realise that when Popper wrote his famous book The Logic of Scientific Discovery [11] (in 1935, though it was not translated into English until 1959), and until 1953, he was a strong advocate of the if interpretation, which he felt to be the standard interpretation for scientists, and the propensity should be seen as opposed to that, rather than a response to the other interpretations of this section. It may even be thought of as a branch or development of the rf interpretation [44, p. 213] or a "virtual-frequency" interpretation [67].

Under the propensity interpretation, Popper sees as fundamental the probability of a result of a single experiment, and sees it as characteristic of the experimental conditions. A probability statement must be tested via a sequence of experiments, but the statement is not about the sequence but about the experimental set-up. Propensities are "tendencies to produce frequencies" [65, p. 33].

Kneale [62, p. 79] suggests that the word "propensity" merely provides a new name for objective probability. Mellor [66, p. 71] agrees that Popper at any rate may be guilty of this approach at times. Where it does go further, the argument may be mainly linguistic [62, p. 81]. Propensity, Mellor insists $[66$, pp. 63, 76] is a disposition, a permanent though latent feature of a situation, which may be displayed by a specific action. Thus electric field, Popper suggests [62, p. 68], is a disposition, since it is a latent quantity, only manifested by the use of a test charge. Indeed Popper [62, p. 70] says that all physical (and psychological) properties are dispositional. A surface is coloured red because it has the disposition to reflect light of the appropriate wavelength. Newtonian mass is the disposition to resist applied force $[66$, p. 79].

Whether such linguistic analysis really solves physical problems may be open to doubt. Popper [62, p. 70] suggests that his interpretation "seems to offer a new metaphysical interpretation of physics (and incidentally also of biology and psychology)". Mellor remarks [66, p. 96], "how much less mysterious death risk and physiological age appear on a propensity than on the received frequency view". Bohm, however [62, p. 79] remarks, "The wave-particle duality is just as difficult when you regard it through propensities as when you regard it any other way." 


\subsubsection{Summary}

One must be impressed by the strength of the belief in individual probability shown by the fact that such a range of vastly talented thinkers (Keynes, Carnap and Popper, for example, must rate among the leading intellectuals of the century) have produced so rich a collection of ideas. One may still be disappointed by the lack of definition, even implicit, of the basic concepts, though this is, of course, precisely what one would expect from section 2.2 .

It is interesting that Mellor [66, p. 2] suggests that the various interpretations of this section are not implacably hostile to each other. His own propensity theory, he says, bases itself explicitly on the interpretation called subjective here, though Mellor calls it "personal", and may have at least a "non-aggression pact" with logical-relation interpretations. To this extent we may regard the interpretations of this section as presenting a united front to the rf interpretation of the following section.

\subsection{Relative-frequency $(r f)$ interpretation}

This interpretation stems from Venn $[68]$ in the last century. The first full discussion was given by von Mises [52], while Reichenbach [48] has provided a more philosophical approach.

In absolute contrast to the prevarication of the interpretations of the previous section, the rf interpretation aims at being totally empirical and giving an explicit definition, related to the relative frequency of occurrence of say, heads in a long sequence of measurements. The layman would certainly accept this as, at least, a good measure of probability, and often discusses probability in such terms. It corresponds to the obvious fact that, to obtain a value for a probability, a number of observations are needed. Many scientists take the same line: Belinfante [69, p. 9] says, "it is qualitatively meaningless to talk about probabilities for a single system". Of course this definition limits the interpretation to cases where a sequence of observations is potentially available, though, from the point of view of this paper. scientific experiments are cases of this sort.

The $\mathrm{rf}$ interpretation is related to those interpretations which recognise individual probabilities by Bernoulli's theorem [46, p. 73]. Contrary to most commentators on this theorem, von Mises [52, p. 108] claims that this theorem is purely arithmetical. It states that if the probability of, say, heads being thrown in an individual trial is $1 / 2$, and all possible sets of results for a sequence of $n$ trials are written out ( $2^{n}$ sets of results), then, as $n$ tends to infinity, the fraction of sequences in which the number of heads is between $n / 2-e$ and $n / 2+e$ tends to unity, where $e$ is an arbitrary small number.

We may argue, then (though von Mises insists that it is an additional assumption) to the usual statement of Bernoulli's theorem: if we study a long sequence of results, we are bound, or practically bound, to find a sequence in which the relative frequency of heads is close to $1 / 2$. (Similar arguments may be constructed for any event with individual probability $p$, the relative frequency also tending to $p$. The von Mises listing will work in an adjusted form if $p$ is rational.)

Von Mises elects to define the probability as the relative frequency in a long sequence. All results of probability calculus, he says $[52$, p. 63], "apply to relative frequencies of events in long sequences of observations, and to nothing else. Thus a probability theory which does not introduce from the very beginning a connection between probability and relative frequency is not able to contribute anything to the study of reality."

Von Mises restricts the type of sequence to those which are random, in the sense that any subset of the sequence will exhibit the same relative frequency. This restriction, however, causes mathematical complications with the idea of the limit [48, p. 148], so Reichenbach dispenses with the idea of randomness, and will analyse any sequence of events. 
We take the opportunity of mentioning the other main area where Reichenbach differs from von Mises. While von Mises states firmly [52, p. 10] that the probability concept cannot be applied to single events, Reichenbach [48, p. 372; 45, p. 161], anxious not to limit the scope of the theory of probability, disagrees. He uses the idea of a posit, used in the sense of a bet or wager: "A posit is a statement with which we deal as true, although the truth value is unknown." We choose a posit which is the best we can make, the term "best" referring "to the posit that will be the most successful when applied repeatedly". (These remarks may be felt to have echoes of the logical relation, the propensity and the subjective interpretation, which may not be too surprising when one recalls the clustering of interpretations allowing an individual nature for probability mentioned in section 2.5.5.)

Reichenbach acknowledges that his solution makes the concept of probability for the single case different from that for the repeatable case. He regards [48, p. 376] the statement about the probability of the single case "not as having a meaning of its own, but as representing an elliptic mode of speech. In order to acquire meaning, the statement must be translated into a statement about a frequency in a sequence of repeated occurrences." It is thus given "a fictitious meaning, constructed by a transfer of meaning from the general to the particular case. The adoption of the fictitious meaning is justifiable, not for cognitive reasons, but because it serves the purpose of action."

For example [48, p. 379], the statement that Julius Caesar was in Britain is regarded as having a weight that is translatable into a frequency statement. "In order to ascertain the reliability of the statement..., we investigate the number of chroniclers who report such a fact; and we measure the reliability of the individual chronicler by the number of his reports that are corroborated by reports of other writers." In other words, the weight of a posit has a statistical origin.

The argument is not very convincing; we must agree with Weatherford [45, p. 162] that if "probability" is defined as "limit of relative frequency in a series", it just is meaningless to speak of the probability of a single event. Nevertheless, the desire of Reichenbach to discuss the probability of a single event, and the reason for this desire, are of considerable interest, and are discussed further in section 2.8 .

Leaving aside this detour, the rf interpretation as described so far does seem clear and appealing. Nevertheless it suffers from grave problems, mathematical and conceptual.

Let us first review the problem raised in section 2.4. In the deterministic case, if one approaches the individual too closely, all probabilities become 1 or 0 . In section 2.5 .2 we saw how the logical-relation interpretation handled the problem. It is just as much a difficulty for the rf interpretation. Let us put ourselves in the position of an insurance company requiring the probability of whether a 40 year old individual will die in the coming year. The rf interpretation will encourage us to inspect a sequence of 40 year old people at times in the past, and find the relative frequency of them dying within the year. But the person we are interested in has a particular sex, so we should in fact construct our sequence only of people of the same sex (as well as of people of the same nationality, of course). He will have a particular medical history, and a particular job, with its own dangers or lack of them, so again our sequence should really only contain people with similar backgrounds. If we move far enough, we must find that our individual is just that, and the sequence is actually null, corresponding to the fact that the probability becomes, as said above, 1 or 0 . For practical purposes, the insurance company must compromise, and accept into its sequence people who have a very general similarity with our individual, but are certainly not identical. The strategy is comparable with that of the logical-relation theory in stating, but limiting, the "evidence" comprising one part of the probability relation.

The mathematical problems of the rf interpretation relate to the connection between probability and relative frequency. The central difficulty $[70$, p. $140 ; 45$, p. 62$]$ is that Bernoulli's theorem does not go 
from probability to frequency, but from probabilities of individual events to probabilities of frequencies of the events. Obtaining a unique rf must, then, be in question.

Bernoulli's Theorem will certainly encourage us to move towards large numbers, where the width of any probability distribution should become smaller, and indeed to the limit of infinite $n$. Indeed nothing other than the limit will do. Reichenbach [48, p. 343] admits, that "Given a relative frequency $f^{n}=m / n$ for the initial section [of a sequence] it is possible to imagine a continuation of the sequence of such a kind that the frequency converges towards any value $p$ selected arbitrarily".

Immediately, though, we note that this is moving away from one of the initial virtues of the $\mathrm{rf}$ interpretation, its empiricism. No sequence of infinite length has been, or ever will be, analysed. Keynes is reported to have remarked on the practical value of the long run justification, "In the long run we shall all be dead." [56, p. 103; 45, p. 217].

More specifically, the mathematics of the limit is complex. Weatherford [45, p. 157] comments that von Mises" definition of probability "abolishes at a stroke every probability theory (practically) from Pascal to Keynes. But it also poses a new and perplexing problem: how does one establish the limiting value of a relative frequency in an infinite empirical sequence?"

The existence of the limit is taken as a postulate by von Mises [52, p. 12]; he calls it his First Law of Large Numbers [52, p. 125]. Jeffreys [47, p. 345] comments that Venn [68] hardly considered it as needing to be postulated! But these approaches cannot be satisfactory. The existence of the limit must be exhibited by pure mathematics. The obvious difficulty is that theorems concerning limits normally consider series in which each term takes a value determined by a mathematical formula. Our case where, at least for von Mises, there is total randomness, is entirely different.

It should be noted that, although Bernoulli's theorem is the source of inspiration for the if interpretation, even to the extent to which it could give us what we require, it cannot be used. As Jeffreys [47, p. 347] points out, the theorem assumes at the outset an individual value for probability. Relative frequentists by definition cannot assume this, and so must tackle a far more general type of sequence.

Initial criticism [71] was largely directed at von Mises' combination of limiting value and randomness; it aimed to show that the two properties were mutually contradictory. Von Mises [52, p. 89] appears to recognise the validity of this objection. Copeland, and von Mises himself [52, p. 229] have attempted to avoid these difficulties, and they were also the reason for Reichenbach dispensing with randomness.

But when this is done, it is clear that there are sequences where the appropriate limit definitely will not exist. Reichenbach himself admits [48, p. 365], "If the sequence under consideration has a limit of the frequency at $p$, it certainly constitutes an admissible interpretation of the statement $P(A, B)$ |the appropriate probability $]=p$. What creates the difficulty, however, is the fact that we must use the sequence as an interpretation before we know whether it has a limit of the frequency at $p$, even before we know whether it has a frequency limit at all. The specific difficulty makes the problem of the application of probability statements unique."

Reichenbach's own solution $[45$, p. 205] is to say that if the limit procedure is never correct, there is no way of making any predictions about the future of the world (that is, of doing science at all). So we must assume its existence, at least in some cases. (Weatherford remarks that short-term predictability would be as useful as infinite-time limits.)

Jeffreys [47, p. 348] believes that the relative frequentists give the whole case away by being reduced to the suggestion that the limiting behaviour is "reasonable". Bunge [44, p. 213] is even more critical: "I do not think one should speak of the frequency definition of probability. There is no such thing - nothing mathematically well-defined, that is. This was shown . . by Jean Ville in his devastating criticism of von Mises' frequency theory of probability ... frequencies are in the bad habit of not being 
perfectly stable: they can jump in outrageously unpredictable ways." One may also note the discussion of von Frassen [67, pp. 181-186], and the remark of Strauss [72] about "the abortive theory of von Mises, and its quick but short-lived success".

Lucas [46, p. 99], on the other hand, suggests that the mathematical difficulties, "although serious, are surmountable... the formulation of a frequency theory may still be possible, mathematical difficulties notwithstanding". But he adds that the frequency theory has in any case lost what seemed initially to be its main advantage, its empiricism, its simplicity as "a layman's theory". He says, "We are no longer being offered a reductive analysis of probability in terms of observed frequencies in actual classes, but a complicated mathematical analysis involving limits, infinities and other highly abstract entities."

We must also take up a specific issue concerning frequencies which are "highly improbable" in the sense that the rf of, say, heads in a sequence of tossings of a fair coin, is very different from $1 / 2$. Let us say we examine sequences in which the rf of heads is 0.9 or higher. The ratio of "successes" to total number of sequences, say $r / 2^{n}$ will tend to zero as $n$ increases, and thus the probability of obtaining this proportion of heads also tends to zero. But this does not mean that $r$ is zero; the unlikely can happen. Lucas [46, p. 99] comments that "such possibilities are difficulties for any theory of probability". However, he says, "the rf interpretation has ruled them out by definition". This, he says, is "fatal", because "definitions are hard, unyielding and brittle ... definitions need to be infallible, or they are futile". The frequency interpretation, he says, is "otiose" because "Bernoulli's theorem already provides a link between probabilities and frequencies" and "wrong" because "it attempts to secure by stipulation what needs to be achieved by honest argument".

As well as these basically mathematical difficulties, the if interpretation has also been criticised on conceptual grounds, specifically that it confuses criteria for the use of a word with its actual meaning, which Weatherford [45, p. 215] calls the Wittgensteinian [73] mistake. Lucas [46, p. 96] compares the confusion with that of the logical positivists who say that the meaning of the statement that Paul is in pain is that he has a drawn face, clenched teeth and so on. This is good evidence that he is in pain, but if we accept this conclusion, Lucas says, we accept something else as well - otherwise, why should we feel sorry for him? Jeffreys [47, p. 379] talks in similar fashion of those who define a colour by its wavelength, those (behavioural psychologists) who define consciousness and thought by throat movements, and Eddington defining time by the direction of increasing entropy. The wavelength, throat movements and so on may be easily observable and thus constitute good evidence for the quantities under discussion, but, Jeffreys suggests, they do not constitute them.

Similarly, these authors suggest, frequencies provide evidence about probability, but they do not constitute it. Bunge [44, p. 214] remarks in similar fashion, "probability is not equal to frequency and it is not even interpretable as such. What is true is that probabilities can be estimated by counting frequencies ... the frequency theories of probability are mathematically nonexistent while the frequency interpretations of it are not exactly true. All we have is a frequency evaluation of probability."

\subsection{Axiomatic method}

An alternative approach to that of the two previous sections should be mentioned. We may introduce it by the remark of Kyburg and Smokler [50, p. 3]: "It has been said (facetiously) that there is no problem about probability: it is simply a nonnegative, additive set function whose maximum value is unity." It is always possible to set up a system of axioms, and explore their consequences, without considering the relation between the mathematical scheme produced and the real world. The leading exponent of this approach has been Kolmogorov [55]. While his work is rigorous and elegant, it leaves 
the question: why should what is defined by his axioms be related to probability, as we usually use the term? Unless an axiomatic approach axiomatises the right concepts, it is fruitless from the practical point of view. For this reason, despite their mathematical importance, axiomatic theories will not be discussed further in this paper.

\subsection{Remarks and conclusions}

The two groups of interpretations seem very opposed - and each has an obvious weakness, in opposite directions. The interpretations of section 2.5 that assign individual probabilities give no clear understanding of the fundamental concepts, though they work coherently with these concepts. On the other hand, the rf interpretation starts off clearly, but rather loses its appeal when its methods are analysed.

Yet, from the point of view of this paper, and in two distinct ways, one should perhaps not overstress the differences. There are two points at which rf interpretations draw back from an even more complete challenge on their rivals.

The first is perhaps verbal, but highly significant. Though relative frequentists obtain values for probability from sequences, in practice they draw back from predicting values only for sequences. We have seen that Reichenbach is willing (elliptically) to give a (fictitious) meaning to the probability of an individual event. And Weatherford [45, p. 176] says that even von Mises allows such a statement as "The probability of drawing an Ace is 1/13." Reichenbach's reason - ". . for the purpose of action" is very important; this procedure allows rf interpretations to stand level with individual probability interpretations for scientific prediction.

The second point again limits the divergence between the two groups of interpretations. Let us imagine light balls being bounced about in a vessel by currents of air. We may discuss the movement of balls either deterministically, or probabilistically. Let us imagine that a particular ball is thought to be registered at opposite ends of the vessel with such a short time intervening that its speed would have needed to be greater than that of light. A deterministic model will reject this. So would a probabilistic model using probabilities for individual events; the probability of this ball crossing the vessel so fast would be zero. The point stressed here is that an rf interpretation would not "hide behind" the fact that only probabilities of groups of particles may be considered, discuss only probabilities of numbers of particles being in different regions at different times, and refuse to reject the proposition that an individual ball could travel in this way. In using an $\mathrm{rf}$ interpretation, we do not give up the right to identify and discuss behaviour of individual systems, using what d'Espagnat [74] calls the "element of reality". We do not really expect different interpretations of probability to give us different physics.

Such appears totally obvious in this highly accessible deterministic model. Yet there may be a message for future sections of the paper. If we argue for ensemble interpretation of quantum theory from rf interpretations of probability, we must be sure that we are not, in fact, smuggling in an extra assumption and effectively denying the "element of reality".

\section{Orthodox interpretations and their problems}

\subsection{What does "orthodox" mean"}

Ballentine [3, p. 360] distinguishes two out of what he calls "many shades" of interpretation. The first, which he supports and terms the statistical interpretation, and which is called here the ensemble 
interpretation, is discussed in the following section. In the second he groups those interpretations which assert that "a pure state provides a complete and exhaustive description of the individual system"; these are what we call "orthodox interpretations", and they are discussed in this section.

Likewise, Jammer [4, p. 440] contrasts the "statistical interpretation" to interpretations in which "the state vector ... is assumed to be the most complete description of an individual physical system". Jammer actually adds to the latter category interpretations "amenable to further completion with at most minor modifications of the formalism, as in the hidden-variable interpretations". In contrast, we follow Ballentine [3, p. 374] in believing that, while the ensemble interpretation makes the search for hidden variables reasonable - different members of the ensemble having different values of the hidden variables - they must be anathema to orthodox interpretations. Indeed Jammer [4, p. 447] suggests that an ensemble interpretation may be seen as the first step to a hidden-variables theory. Thus we take interpretations involving hidden variables as variants of ensemble interpretation.

Another grouping is made by Murdoch [75, p. 109]. He describes the "statistical interpretation", in which the state vector represents "the state of a statistical ensemble", the "ontic interpretation" in which it represents "the real physical state of an individual object", and the "ensemble interpretation", in which it represents the "state of knowledge of an object".

His first two interpretations clearly correspond to those of Ballentine and Jammer, though Murdoch's reference to "real physical state" may go further than these authors would wish. Murdoch's third category also is recognised by Ballentine, who disregards it. He comments $[3, \mathrm{p}$. 361] that one cannot deduce new knowledge from lack of knowledge, and [3, p. 371] that study of a person's knowledge of physics is very different from study of physics itself. Murdoch himself would not treat the epistemic interpretation so lightly, and his ideas will be returned to at various points in this review.

While the different definitions of what we call the orthodox interpretation given by Ballentine and Jammer (in particular the shades of meaning to be attached to the word "complete") will also be discussed later, in practise it is clear to whom they are referring. While Ballentine $[3$, p. 360] includes Schrödinger's original electromagnetic interpretation [76], not now considered tenable (though conceivably open to revival [77]), he mainly targets "the several versions of the Copenhagen Interpretation". He makes, though perhaps understates, a crucial distinction between what he calls the Copenhagen school, and the Princeton school, both of which "claim orthodoxy".

The Copenhagen school was, of course, centred round Bohr. Bohr's approach was principally conceptual rather than mathematical. As Jammer [4, p. 472] and Murdoch [75, p. 109] point out, he never discussed the theory of measurement in formal terms, despite its central role in his philosophy of physics. Rather, he provided, initially in his Como lecture of 1927 [78] and in much subsequent elaboration, and under the name of complementarity, a general conceptual framework which he hoped would (in the words of Folse) "resolve all the apparent inconsistencies ensuing from the attempt to work the quantum postulate into the classical framework" [79, p. 104]. (Indeed, he hoped it would go further and teach "an 'epistemological lesson' pertinent to all description of nature" [79, p. 1]; Bohr himself attempted application to psychology and biology [79, ch. 6].)

Other important figures associated with the Copenhagen school were Heisenberg, Pauli and Rosenfeld. Heisenberg's philosophy $[80,81]$ was perhaps less consistent than that of Bohr; Ballentine $[3$, p. 360$]$ says that some of his pronouncements could well support an ensemble interpretation, while others would be clearly opposed. We shall give a brief quotation from the work of Heisenberg [81, p. 179] which reflects most clearly one way in which Heisenberg's ideas depart from those of Bohr: "[T]he concept of complementarity introduced by Bohr into the interpretation of quantum theory has encouraged the physicists to use an ambiguous . . . language, to use the classical concepts in a somewhat vague manner in conformity with the principle of uncertainty ... When the vague and unsystematic use 
of the language leads into difficulties, the physicist has to withdraw into the mathematical scheme and its unambiguous correlation with the experimental facts". As will be made clear, we do not believe that Bohr would support these views at all. Pauli's ideas have probably received less attention than they deserve; Laurikainen [82, p. 273] has recently stressed his divergences from Bohr and Heisenberg. Rosenfeld, disciple and, for many years, close collaborator of Bohr, became the leading advocate for complementarity for a considerable period $[62, \mathrm{p} .41]$.

It is also appropriate to include here the name of Born, the only other of the main founders of quantum theory to endorse fully the Copenhagen position. It was Born, of course, who introduced the idea of probability into quantum theory in his initial paper on collisions [83]. This idea cannot as such, of course, be considered as a contribution to any particular interpretation of quantum theory. Rather. as Beller [84, p. 563] puts it: "Together with Heisenberg's indeterminacy principle, it belongs to the noncontroversial fundamentals of quantum mechanics."

In fact we would not regard Born as a major contributor to the Copenhagen interpretation. Beller [84] has given an interesting account of the development of his ideas through the crucial period. She emphasises that, at this time "all of Born's intellectual pronouncements were fluid and uncommitted" [84, p. 564], and, indeed, that as "a mathematical man", it was natural for Born "to introduce formal symbolic solutions with the minimum of interpretational commitments." [84, p. 569].

Initially it seems [84, p. 566] that Born was very much influenced by Schrödinger's interpretational emphasis on representing electrons as waves in real space, with the elimination of quantum jumps. He considered Schrödinger's wave theory "physically more meaningful than matrix mechanics" (of which, he was, of course, one of the founders) [84, p. 570]. In keeping with the stress on waves, the wave function was not initially connected with probability of position; it controlled only "the [probabilities of] energetic transitions of an atom and the energy and direction of motion of colliding electrons" [84, $\mathrm{p}$. 571]. In his next papers $[85 ; 84$, p. 575], Born extended the domain of probabilistic ideas to stationary states; he proposed that $\left|c_{n}\right|^{2}$ gave the probability that the system was in the stationary state $\psi_{n}$. His model was of stationary states between which transitions took place.

The final step was taken by Pauli $[86 ; 84$, p. 575]. While Born still only regarded it as meaningful to discuss probabilities of the energies and angular momenta of stationary states, Pauli proposed that $|\psi|^{2}$ was a probability density, i.e., $|\psi|^{2} \mathrm{~d} x$ was a probability of position. As Beller points out $[84, \mathrm{pp} .571$, 575], this concept was likely to be uncongenial to Born and Heisenberg, founders of matrix mechanics. which was "erected on the basis of and with full conviction in the bankruptcy of corpuscularkinematical models of an atom".

It was also far removed from Schrödinger's views which allowed no particle-like notions such as position. By this time, in any case, Born had become much opposed to Schrödinger's interpretational views, partly because he felt one must retain the concept of the quantum jump, conclusively because, with the development of transformation theory, it became clear that a (Schrödinger) description in real space may be transformed to a description in (for example) momentum space, so Schrödinger's wave mechanics is just "a mathematical appendix to matrix mechanics" [84, p. 572]. Born thus "allied himself with Schrödinger's adamant opponents", Heisenberg and Jordan.

Beller stresses, however [84, p. 573], that Bohr took a less partial view; he "did not agree to the Göttingen total depreciation of physical meaning of Schrödinger's theory, proposing his complementarity as a compromise". This merely returns us to our initial point; though the ideas of such as Heisenberg, Pauli and Born were important in the process of dialogue leading to complementarity and the Copenhagen interpretation, it is still appropriate to regard Bohr as virtually the sole progenitor of the position itself. 
Von Neumann was the founder of what Ballentine calls the Princeton school. We would prefer to call it the Princeton interpretation, distinct from, rather than part of, the Copenhagen interpretation. This agrees with Feyerabend [87, p. 237], who suggests, "when dealing with von Neumann's investigations, we are not dealing with a refinement of Bohr - we are dealing with a completely different approach".

In his famous book [14], the main and highly important achievement of which was to provide a rigorous foundation for quantum theory based on Hilbert space, von Neumann also investigated in detail what he called $[14$, p. vii] "the difficult problems of interpretation, many of which are not even now fully resolved". As well as his claim to show that hidden variables could not exist, this involved a formal theory of measurement centred on the projection postulate, the idea of collapse of state vector at a measurement. As discussed in section 3.2, Dirac and Wigner may be said to have contributed to this type of interpretation.

In 1970, Ballentine [3, p. 360] apparently considered the clash between Copenhagen and Princeton to be a recent difference of opinion between groups supporting Rosenfeld and Wigner. He did not seek to differentiate their opinions, though his own account of the Copenhagen approach to measurement was largely that of von Neumann.

Shortly afterwards, a paper by Stapp [88] appeared, attempting to clarify the essence of the Copenhagen interpretation. Stapp stated categorically that what he called the absolute- $\psi$ approach of von Neumann was in many ways directly opposed to the true Copenhagen interpretation (that is, the approach of Bohr), and his views were endorsed in letters from Heisenberg and Rosenfeld published in an appendix.

Ballentine [89] has suggested that, in this paper, "in attempting to save "the Copenhagen interpretation", Stapp "radically" revises what is often, rightly or wrongly, understood by that term". He calls Stapp's views "the neo-Copenhagen interpretation", and agrees that he and Stapp "are in substantial agreement about the concept of "state"".

In this section of the paper, we study the views of von Neumann (in section 3.2) and Bohr (in section 3.3), together, to an extremely limited extent, with the approaches of their supporters and commentators. (The reason for the reversal of historical order is that, because of the comparative simplicity, at least in motivation, of von Neumann's views, it is convenient to introduce them before those of Bohr.) Following Ballentine, both of these authors are being considered as advocates of the "orthodox" interpretation, in which a wave function represents a single system. In fact, in both cases this point has been questioned.

On von Neumann, Jammer [4, p. 443] considers that "the general tenor" in which his work was written is "of the statistical ensemble interpretation", and that "his approach to the mathematical treatment of quantum phenomena often cries out for such an interpretation". He also reports similar remarks from Blokhintsev. Jammer admits, though, "von Neumann seems never to have committed himself verbis expressis to the statistical view", and, "his endorsement of the Bohr and Heisenberg thought experiments" conflicts with this general approach.

In the case of Bohr, Teller [90] states his position that "very simply . . Bohr gives the state function a statistical interpretation".

Murdoch's views are also of interest. Of his three interpretations of the state vector mentioned above, he believes that Bohr was "chary of the statistical interpretation" [75, p. 119], though he, in fact, "recognised, quite correctly, that the state vector could be interpreted either in statistical terms or in epistemic terms, and he believed that for practical purposes it was immaterial which interpretation one adopted". According to Murdoch, though, Bohr considers the epistemic interpretation was "less misleading concerning the question of completeness", and Murdoch suggests that all the evidence is 
against Bohr adopting the ontic position [75, p. 122]. Murdoch provides his own formal version for Bohr's theory of measurement, although, as we have already seen, Bohr refrained from giving such a formal account; while the ideas, based on what Murdoch calls the objective-values theory of measurement, and discussed in section 3.3.3, are not simply categorisable, they certainly go beyond what we have called here the orthodox position.

We see, then, that the demarcation between "orthodox" and "ensemble" interpretations is nowhere near as clear-cut as Ballentine may have supposed. This point will re-emerge in section 5 .

\subsection{Von Neumann and the Princeton interpretation}

\subsubsection{The projection postulate for observed system}

Von Neumann's approach may be described as formal and mathematically compact. In contrast to that of Bohr, it is not derived from rigorous conceptual analysis; rather it responds internally to conceptual problems posed by the approach itself. Its workings are formally fairly straightforward, because, again in contrast to Bohr, it treats the measuring device quantum mechanically. Its basic simplicity practically forces it into the projection postulate.

As Sudbery [91] points out, this postulate was certainly not first stated by von Neumann himself. Dirac, in his famous book [92, p. 36] had already said, "a measurement always causes the system to jump into an eigenstate of the dynamical variable that is being measured, the eigenvalue this eigenstate belongs to being equal to the result of the measurement". This in turn only repeated remarks he had already made at the 1927 Solvay Congress [93, p. 262]. Neither was Dirac necessarily the inventor of the concept [94]; many physicists probably developed similar ideas at about the same time.

More specifically the "jump" or "collapse" may be written as follows [4, p. 475], conveniently using the density matrix; $\rho \rightarrow \rho^{\prime}=\Sigma_{i} P_{i} \rho P_{i}$, where $\rho$ is the density matrix before collapse, and the $P_{i}$ are the projection operators onto the eigenvectors of the observable being measured. In general (unless $\rho$ represents a pure state which also happens to be an eigenstate of $\hat{O}$, the operator representing $O$, the observable being measured), the state after collapse will be a mixture. This corresponds to the fact that, at a measurement, one specific result is obtained, $O_{j}$ with probability $\left|\left\langle\phi_{j} \mid \psi\right\rangle\right|^{2}=\left|c_{j}\right|^{2}$, where $|\psi\rangle$ is the initial state vector, $\left|\phi_{j}\right\rangle$ is the eigenstate corresponding to eigenvalue $O_{j}$, and $|\psi\rangle=\Sigma_{j} c_{j}\left|\phi_{i}\right\rangle$. (In writing a state vector for the initial state of the system, we are assuming it to be pure; if it is not, we may sum over different initial pure states.) If the result $O_{i}$ is obtained, the projection postulate tells us that the collapse is to state $\left|\phi_{j}\right\rangle$.

Such a statement is, of course, unambiguous only if $\left|\phi_{j}\right\rangle$ is nondegenerate, but a suitable generalisation has been constructed by Lüders [95].

To both Dirac and von Neumann, the postulate apparently seems unavoidable. For von Neumann [14, pp. 347, 418], it almost seems no more than a statement of what a measurement is. Dirac [92, p. 36] introduces the idea of the repeated measurement. "From physical continuity", he says, "if we make a second measurement of the same dynamical variable immediately after the first, the result of the second measurement must be the same as that of the first ... Hence, after the first measurement has been made, the system is in an eigenstate of the dynamical variable [measured], the eigenvalue it belongs to being equal to the result of the first measurement."

Postponing for a little discussion of their arguments, we immediately note that the projection postulate has been the subject of an immense amount of analysis and criticism. The difficulty is that, in von Neumann's words [14, p. 417], there appears to "a peculiar dual nature of the quantum mechanical procedure" which could not be satisfactorily explained. (This is often called the "measurement 
problem" of quantum theory.) In the absence of any measurement, a quantum-mechanical state develops according to the Schrödinger equation (a process of type 2 according to von Neumann); at a measurement, it follows the projection postulate (a process of type 1).

The difference is fundamental. In a process of type 2 , an initial pure state remains pure; von Neumann calls such a process causal. In a process of type 1 , it becomes, in general, a mixture, and von Neumann calls such a process noncausal. Sudbery [91] points out that, if the initial state is pure, a type 2 process maintains the rank of a density matrix, while a type 1 process changes it. Von Neumann [14, p. 379] has shown that a process of type 2 is thermodynamically reversible, while one of type 1 is irreversible.

Despite this apparent dichotomy, there have been innumerable attempts to demonstrate that a process of type 1 can be approximately, or in some limit, equivalent to one of type 2, or, more exactly, that the results of processes of the different types may be indistinguishable. In order to consider these arguments, we need to introduce explicitly the measuring apparatus or the observer. So far, following Dirac, and von Neumann's initial considerations, we have restricted ourselves to analysis of the measured system only. Clearly, though, a measurement requires the use of a measuring apparatus, and the hallmark of a measurement is an irreversible process, either in the apparatus itself, or in the memory of an observer. In section 3.3. we shall see that for Bohr the interaction between observed and observing systems is central to solving the problems of quantum measurement. For von Neumann, too, explicit consideration of the measuring apparatus clarifies the problems, though it does not really solve them, and we shall follow this path in the following two subsections.

Let us first, though, at least raise the question of whether the problems are just as severe as so far suggested. The mode of description has been that the state vector is very much a "property of the system", analogous to position or velocity for a classical particle. If it is found to change, then, just as for the position or velocity of a classical particle, there must be a specific physical cause. Certainly this approach works well for processes of type 2 , but leaves the problem of measurement as already outlined.

This approach is what Stapp [88] denounces as the absolute- $\psi$ interpretation. It also seems to be Murdoch's ontic interpretation of section 3.1, with its insistence on representing the "real physical state" of a system. There are other approaches to the state vector that make it easier to understand the occurrence of processes of both types. These include Murdoch's epistemic interpretation, where naturally knowledge of a physical situation is changed by a measurement. They also include interpretations where the state vector is related to probabilities of occurrences (rather than merely knowledge of such), but without the additional suggestion that it is a "property of the system". (Such approaches may be thought of, at least loosely, as intermediate between Murdoch's ontic and epistemic interpretations.) Again probabilities of later occurrences will be altered by earlier occurrences, so it is reasonable that a state vector should change at a measurement. These kinds of interpretation require both types of process, but do not require processes of type 1 to reduce down to those of type 2 . This is not so say, of course, that they are necessarily internally consistent; they may be avoiding the real issues altogether, as Ballentine's comment on the epistemic interpretation quoted above suggests.

\subsubsection{Some views on measurement}

We now return to Dirac's argument for the projection postulate from the idea of repeated measurement. This has been the starting point for much discussion of the concept of measurement.

Landau and Peierls [96] criticised the assumption that a repeated measurement will, in general, yield the same result. They pointed out that in classical physics, where $h$ may be taken as zero, the states of 
the system can be assumed to be identical. However in quantum theory this is not the case, because the measurement causes a change in the system which is in principle impossible to determine. They distinguished two types of measurement. A measurement of the first kind determines the state of the system after the measurement is made, and the measurement is repeatable. A measurement of the second kind is not repeatable; it examines the state of the system which existed before the measurement took place. Pauli [97] more specifically demands that measurement changes the state of the system in a controllable way so that the value the observable had before the measurement may be deduced unambiguously. Thus determination of an atomic energy level via an electron scattering is a measurement of the second kind.

Landau and Peierls distinguish repeatability from predictability. The latter implies that for any measuremental result, there is a state of the system in which the measurement certainly gives that result; the existence of predictable measurements, they say, is an absolutely necessary condition for the validity of wave mechanics. Repeatability, though, they claim, is only achieved for measurements of an observable if the corresponding operator commutes with the energy of interaction between system and apparatus; this limits repeatable measurements to those of the position coordinate. (See the related comment in the textbook of Landau and Lifshitz [98, p. 24].)

Margenau [99] took the questioning of the simple Dirac approach further. First he cast doubt on the whole idea of repeated measurement. Against the specific proposal of Einstein that a photon passing through one polariser will certainly pass through a second polariser with the same orientation, he pointed out that this is definitely not a repeated measurement. "Neither of the two acts constitutes a measurement unless the hypothecated passage of the photon is documented by the emergence of a 'number', yes or no, and this is usually done by observing the photon after passage through the polarizer, i.e., by allowing it to be absorbed... If we make the first polariser into a measuring apparatus by adding a photocell, the second will perform in general no measurement at all. If we supplement the second one in this manner, the first polarizer merely prepares a state."

The last words signify the terminology introduced by Margenau. Passing a photon through a polarizer is not a measurement; it is a state preparation, preparing systems in a reproducible state. As Ballentine points out [3, p. 366], the concept of state in quantum theory may be regarded as an abbreviation for a (probably not unique) state-preparation procedure. A measurement, on the other hand, requires an irreversible coupling between the system and a measuring device that produces a permanent measuremental result. A measurement implies detection, while a state preparation provides information about the system if it passes through the apparatus. Thus a measurement of the first kind, in the sense of Pauli, is a measurement and a state preparation.

As Margenau [99] points out, a measurement quite often annihilates the system being measured, for example the detection of the photon by the photocell above, or a position measurement of any particle by recording on a photographic plate. Thus, in these cases at least, the question of repeated measurements and the projection postulate does not arise.

This clarification is useful; it does not help us, though, to relate the result of a measurement to any property of the system before measurement. Ballentine [3, p. 366], and Murdoch [75, p. 105] attempt to answer this question by defining a measurement as giving us information from which "we may infer the value of [the measured quantity] (within some finite limits of accuracy) which the system had immediately before the interaction [between system and apparatus] (or the value which the system would have had if it had not interacted)" (in Ballentine's words); "what we actually observe is the property of the object before it is disturbed" (according to Murdoch). Thus according to these authors, any measurement is of the second kind in Pauli's terminology. 
Arguments for this position may be of two types. The first is semantic. If a process was not of this type we would not call it a measurement. Murdoch bases his argument here firmly on his reading of Bohr, and we study it in section 3.3.3. Initially [75, p. 105], in emphasising Bohr's aversion to such phrases as "creation of physical attributes to objects by measurements", Murdoch does not make quite clear whether he disapproves equally of two distinct possibilities. In the first, the measurement changes the value of the measured property from one value to another, and the measured value is other than the initial value; it could be the final value (making the measurement of the first kind, in the terminology above) or some other value. In the second possibility, the observed property literally has no value until the measurement; the measurement provides it with a value, at least as a measuremental result, though there is no guarantee that, at the conclusion of the measurement, the property has this or any other value.

Later, however, Murdoch [75, p. 128] specifically rules out both: "In quantum physics terms such as 'observation', 'attribute' have their ordinary language meanings, which do not imply that observation creates the observed property, or that the observed property is a 'disturbed' property."

More direct argument comes from analysis of actual experimental procedures by such writers as Park and Margenau [100] and Ballentine [3, p. 365]. These (thought)-experiments measure the position coordinate of a particle directly, but also claim to use this measurement together with previous information on the system to obtain a value for a component of the momentum in the period before the measurement. If their case is accepted, these experiments show that the uncertainty principle in at least one form - that simultaneous measurements of observables whose operators do not commute cannot be made - does not hold for this type of measurement, though it certainly holds for state preparations.

Such experiments were indeed considered by the founders of the orthodox interpretations of quantum theory. Heisenberg [80] discusses them, and suggests that "the uncertainty relation does not refer to the past". But he says, "this knowledge of the past is of a purely speculative character", and adds, "it is a matter of personal belief whether such a calculation concerning the past history of the electron can be ascribed any physical reality or not." Popper [65, p. 27] laconically comments that "almost every physicist who read Heisenberg opted for "not"".

Actually, supporters of orthodox interpretations needed to follow this line, for otherwise they would be denying their central premise - that the state vector contains the most complete possible description of the system. Were they justified in so doing? After all, as Ballentine points out, the kind of argument used by those suggesting these thought-experiments follows directly from classical ideas. Yet it has already been mentioned that classical measurements do have different features from those dealing with microscopic objects [96]. Orthodox interpretations must say that, prior to a measurement, the properties of a system just do not exist (except, if one wishes, one may consider values for observables, if the state vector of the system is an eigenfunction of the appropriate operator).

As an example, imagine a particle emitted at the origin at $t=0$, and an array of detectors over a sphere centred at the origin. Prior to detection, orthodox theory at least would say that the components of momentum just do not exist. After detection, at a particular point, one can, of course, argue back and produce a path, but, as Heisenberg suggests, one should be extremely dubious about attributing any reality to it.

Vigier [82, p. 653] describes a position, which he attributes to Bohr, as follows: "The fundamental unit for description is the whole 'phenomenon' constituted by object and apparatus ... All atomic phenomena are closed in the sense that their observation is based on registrations obtained by means of suitable amplification devices with irreversible functioning which define the limits of objective description. Since such closed quantum phenomena may not be further analysed, what happens inside or 
between such phenomena, the "interphenomena', cannot be conceived in quantum description." (It should be mentioned that Vigier himself opposes this position.)

Such is the kind of position that orthodox interpretations are pushed towards. Supporters of ensemble interpretations, like Ballentine, Popper and Margenau, would dismiss it, and regard their thoughtexperiments as some of the best arguments for their own interpretations. One should again mention the rather different position of Murdoch [75] discussed in section 3.3.3.

To conclude this section, we mention two recent points made by Bell [101]. The first refers to the question of repeatability and the arguments of Landau and Peierls above. Bell points out, "in physics the only observations we must consider are position observations, if only the positions of instrument pointers". For example, the so-called measurement of the energy of an atom via scattering of an electron, mentioned as an example of a measurement of the second kind, should really be regarded as an observation of the position of the electron, from which observation, information about the atomic system may be deduced. Measurements of position, of course, are precisely those that we expect to give the same result if repeated, from relativity considerations alone, and this appears to support the projection postulate, though, of course, this cannot refer to primary measurements which destroy the particle observed.

Bell's second point, admittedly semantic, concerns "the pernicious misuse of the word 'measurement' in contemporary theory" which "very strongly suggests the ascertaining of some pre-existing property of some thing.". Bell is "convinced that the word "measurement' has been so abused that the field would be significantly advanced by banning its use altogether in favour for example of the word "experiment"".

Such a procedure would make irrelevant some of the more verbal sparring, described in this section, allowing attention to be focused on the really important issue, the existence or otherwise of values of position and momentum at all times.

\subsubsection{The projection postulate - inclusion of observing system}

We have seen that, in several interpretations, inclusion of the observing system is considered essential. For von Neumann it is not obligatory, but the principle of psycho-physical parallelism, which von Neumann [14, p. 419] calls a fundamental requirement of the scientific viewpoint, does impose restrictions. In any measurement, at one end we have a physical system - von Neumann's example is a system whose temperature is to be measured; at the other end, there is what von Neumann calls the "ego" of the observer. In between are the thermometer, some light quanta which travel from thermometer to retina, the optic nerve tract, and the brain.

In any analysis of the measurement, there must be a cut between what is treated as physical, and what as psychological; in other words, between "observed" and "observing" systems. Psycho-physical parallelism tells us that the cut may be made at any point in the chain, and its position must not affect the results obtained. (At this point in his argument, von Neumann acknowledges Bohr [102]; however in general his approach is so different that, as stated in 3.1, we do not consider von Neumann an authentic representative of the Copenhagen interpretation.)

The treatment of section 3.2.1 applies to any initial positioning of the cut. Von Neumann [14, p. 422: 4 , p. 476] then shows that, if any portion, $\beta$, of what is initially the observing system, adjacent in the chain to the initial observed system, $\alpha$, is instead included in the observed system, it is possible to find an interaction Hamiltonian between $\alpha$ and $\beta$ which builds up a one-to-one correlation between states of $\alpha$ and states of $\beta$. Instead of $\left|\psi_{\alpha}\right\rangle=c_{i}\left|\phi_{j \alpha}\right\rangle$, we have 


$$
\left|\psi_{\alpha}, \psi_{\beta}\right\rangle=c_{j}\left|\phi_{j \alpha}, \phi_{j \beta}\right\rangle
$$

where $\left|\phi_{i \beta}\right\rangle$ is a state of the observing apparatus corresponding to state $\left|\phi_{j \alpha}\right\rangle$ of the observed system, and $c_{i}$ is unchanged from section 3.2.1. Thus an observer of $\beta$ rather than $\alpha$ will find the probability of observing $O_{j \beta}$, the eigenvalue for $\beta$ corresponding to eigenstate $\left|\phi_{j \beta}\right\rangle$, is $\left|c_{j}\right|^{2}$, as in section 3.2.1.

This argument shows that von Neumann's arguments agree with his principle of psycho-physical parallelism. The cut between "observed" and "observing" systems may be made where we choose, and the results are unaffected.

The consistency of the formalism only serves to re-emphasise the problem of quantum measurement as described in section 3.2.1. The right-hand-side of (3.1) is still a pure state, a linear combination of coupled states of observed and observing systems. To be sure $[14$, p. 429; $103 ; 74 ; 4$, p. 479], an operator $T_{\alpha}$, corresponding to observed system $\alpha$ only, will have zero matrix elements between states $\left|\phi_{j \alpha}, \phi_{j \beta}\right\rangle$ of differing $j$, so the linear combination may be regarded as an "improper mixture" in the terminology of d'Espagnat. But the system cannot be left in a linear combination; von Neumann insists that a measuremental result must be a single $\left|\phi_{j \alpha}, \phi_{i \beta}\right\rangle$. Equally, such procedures as observing state $\beta$ only introduce further links in the chain, the end of which is still a pure state, not a genuine mixture. (And von Neumann [14, p. 437] shows explicitly that the inherently reasonable suggestion that the initial state of $\beta$ might be a mixture does not solve the difficulty.)

Von Neumann's solution [14, p. 418] is that the final stage of the chain must be entirely different from all previous stages. He says, "it is inherently entirely correct that the measurement or the related process of the subjective perception is a new entity relative to the physical environment and is not reducible to the latter. Indeed, subjective perception leads us into the intellectual inner life of the individual which is extra-observational by its very nature". Von Neumann adds, "experience only makes statements of this type; an observer has made a certain (subjective) observation; and never any like this: a physical quantity has a certain value". So, although the cut between observed and observing is movable, a cut there must be.

Thus responsibility for collapse is very much put onto the observer - the "abstract ego" (not merely in the capacity of the observer as a macroscopic object - as noted, other macroscopic objects, measuring devices, pointers and so on, are considered to possess state vectors, and we may consider linear combinations of different macroscopic states).

For von Neumann, this appears implicit in what a measurement is. Wigner [104] too suggests that it is "consciousness" that collapses a state vector, but for him, rather than this being of the nature of things, a more specific model is put forward. It is suggested that consciousness adds a nonlinear term to the Schrödinger equation.

\subsection{Bohr, Copenhagen and complementarity}

\subsubsection{Initial comments on Bohr}

For many years most texts on quantum theory have routinely acknowledged that they were following the Copenhagen interpretation, and advocated the principle of complementarity $[105$, p. $27 ; 106$, p. 8], yet discussion and explanation have usually been minimal. In fact the issues are far from simple, and Jammer shows $[4$, p. 88$]$ not only that Einstein, leading anti-complementarist, was unable to achieve a sharp formulation of the principle, but von Weizsäcker, great supporter of Bohr, struggled to obtain a clear understanding of complementarity, an understanding which Bohr immediately rejected. Hence we 
shall not aim in section 3.3.2 at a complete account, but merely attempt a general discussion which brings out the main points.

One reason for the difficulty found in understanding Bohr has already been mentioned. Although measurement was central to his ideas, unlike von Neumann he never suggested a formal theory. (Murdoch [75] has attempted to fill the gap; his ideas have been touched on in section 3.2.2 and will be considered more fully in section 3.3.3.)

Another problem is that Bohr's position has very often been taken to be, either deliberately on his part, or in effect, merely one of the major schools of philosophy. Folse [79, p. 18] writes, "he has been championed as a positivist, a realist, a materialist, an idealist, and a pragmatist, and at the same time criticized under at least as many labels". We saw in section 1 that he has been denounced for idealism. The attacks of Popper $[65$, p. 7] and Bunge $[65$, p. 105] accused him chiefly of subjectivism (in that the observer played a role in measurement). However the modern fashion, in semipopular works in particular, is to describe Bohr as a positivist (with possibly tinges of operationalism or instrumentalism). Just as an example, a recent biography of Dirac [107, p. 80] mentions "the positivist and instrumentalist attitude of the Copenhagen-Göttingen camp".

To explain the difficulties in quantum interpretation on the basis of these philosophies would appear relatively simple, at least in outline. One would concentrate on the prediction of experimental results. Deeper speculation could be dismissed, positivistically, as meaningless; concepts could be defined, operationally, only through their measurement, or nonobservable theoretical concepts could be regarded, instrumentally, as potentially useful, but disposable. Such positions appear fairly clear-cut, and clearly a priori philosophically, and independent of any experimental result.

Bohr's position was entirely different, being wholly founded on experimental results, in particular the necessity to interpret experiments such as the photoelectric effect by making the quantum postulate and assuming a quantum of action. His views were built up by sustained conceptual analysis.

Folse [79, p. 236] comments, "Bohr could have totally avoided all of the torment which he experienced in grappling with the wave-particle dilemma by simply adopting an instrumentalist interpretation of quantum theory. Indeed many have assumed this is just what he did. But the history ... tells a different story. Given his conviction that the new framework must revise the classical account of descriptive concepts, it was only natural that instead he should elect to redefine the relationship between the concepts used in a theory and the phenomena which confirm that theory. Had he simply adopted instrumentalism as a way out of wave-particle dualism, there would have been no need to revise the classical framework." Folse continues, "He could have kept the classical framework and merely discarded the realist interpretation of the description of nature within that framework. But in fact he did the opposite; he discarded the classical framework and kept a realistic understanding of the scientific description of nature. What he rejects is not realism, but the classical version of it."

Since Bohr's position is one of considerable subtlety, and is not always presented clearly, it is not surprising that it has been simplified or misrepresented. It is good, therefore, that two recent studies by Murdoch [75] and Folse [79] have undertaken to provide sustained analysis of Bohr's views based on the writings as a whole rather than individual remarks.

Such study shows that, although Bohr's ideas may have had common features with some of the prevailing philosophical tendencies, and could be construed in places as congruent with such, Bohr's starting point and general aims were very different. A quantity was not dismissed as meaninglass merely because it was not accessible to measurement. Though, as we shall see, a physical quantity could only be considered to have a specific value in an appropriate measuremental setting, it was not defined by the measurement. 
Indeed, while the popular view of Bohr today may be of a nonrealist, both Folse (as already suggested) and Murdoch are prepared to give Bohr the title of realist. For Folse [79, p. 20], "complementarity offers a realist interpretation of scientific theory in the sense that it provides knowledge about what it takes to be independent existing real atomic systems and their constituents" even though "Bohr definitely argues against the form of realism defended by Einstein". Again, "Bohr never argues that the notion of the object as an independent reality is meaningless" [79, p. 165]. Folse suspects [79, p. 23], "The nature of the philosophical debate between realism and antirealism lay essentially beyond Bohr's intellectual horizons." Bohr "took it as empirically demonstrated that atomic systems were real objects which it was the goal of an acceptable atomic theory to describe". For Bohr $[79$, p. 243] reality must be assumed, not demonstrated empirically.

Murdoch is perhaps a little less convinced, but is prepared to describe Bohr as an "empirical realist" $[75$, p. 210$]$ or a "weak realist" $[75$, p. 216$]$.

These comments, as explained, refer to the whole range of Bohr's work. A clear specific example of Bohr's approach would be in his contribution to the Schilpp volume on Einstein [18, p. 199], where his unqualified talk of "objects" demonstrates his unquestioning acceptance - naive, perhaps, from the philosophical point of view - of the existence of atoms and their constituents.

What Bohr put forward was not a (perhaps arbitrary) philosophical position, but an understanding of how our conceptual framework must be adjusted, in fact limited, to meet new experimental facts. He claimed, "the viewpoint of complementarity may be regarded as a rational generalisation of the very idea of causality" [18, p. 211]. Folse says, "complementarity is an attempt to reckon with new physical facts and yet preserve objective description" [79, p. 221]. We shall sketch this attempt in the following section 3.3.2.

While we have rejected claims that Bohr is positivist, instrumentalist and so on, much more careful analysis has been performed which claims to demonstrate his support of the "pragmatism" of William James, and, in particular, Bohr's compatriot and friend, Harald Høffding. Murdoch [75, p. 224] suggests that, in Bohr's view, "The ascription of an exact position and an exact momentum to an object at the same time is meaningless, not principally because such a property is unobservable, but because the ascription has no practical consequences whatever: it has no explanatory or predictive power ... The fact that the statement is not verifiable is only one factor among others that makes it meaningless to assert it. The meaning conditions, then, are not primarily epistemic, but pragmatic, having more to do with what we as agents can do than with what we can know." Stapp [88] bases his account of the Copenhagen interpretation on the idea that "quantum theory is fundamentally pragmatic, but nonetheless complete". These authors certainly show that Bohr found the pragmatic position congenial. Nevertheless, one may hesitate to endorse fully such comments as that of Stapp that "the Copenhagen interpretation .... is fundamentally a shift to a philosophical perspective resembling that of William James" if it implies an initial taking up by Bohr of a philosophical position. As Folse says [79, p. 154], "Bohr bases his argument on ... empirical discovery of atomic physics: that expressed in the quantum postulate". Complementarity may be able to coexist with pragmatism but it did not have its agenda set by it. Rather, it was based firmly on experiment in the form of the quantum postulate.

\subsubsection{A sketch of the complementarity argument}

The quantum postulate - the fact that any interaction between two atomic objects, or an atomic object and a measuring device, must be discontinuous-means that the state for each of the participating systems cannot be defined, as one would expect classically, only a combined state for the whole system. 
If we concentrate on the measurement case, it is clear that the classical picture must be relinquished. Classically, observed and observing systems interact, but the effect on observed system is either negligible, or, because of the continuity of interaction, may be calculated. Also, as Folse [79, p. 110] makes particularly clear, discussion in terms of observing system disturbing observed system in an uncontrollable way, is not adequate. Such a description would allow one to conceive of systems existing in classical states, which one could not observe because of "observational disturbance". As Bohr pointed out in his original Como article [78], the description is inadequate because (see section 3.2.3) the boundary between observed and observing systems is arbitrary. The interacting system must be considered as a whole, not as two components between which a noncalculable discontinuous interaction takes place.

Later [18, p. 209], he wrote, "this crucial point . . implies the impossibility of any sharp separation between the behaviour of atomic objects and the interaction with the measuring instruments which serve to define the conditions under which the phenomena appear".

Such an argument might well seem to inhibit one from describing the results of measurements in any way comparable to that used in classical physics. Yet the second great starting-point for complementarity is that such a description is the only one open to us. Bohr writes, "our interpretation of the experimental material rests essentially on the classical concepts" [78]; and "however far the phenomena transcend the scope of classical physical explanation, the account of all evidence must be expressed in classical terms" [18, p. 209].

In a letter to Schrödinger in 1935 , Bohr wrote, "my emphasis on the unavoidability of the classical description of the experiment refers in the end to nothing more than the apparently obvious fact that the description of every measuring apparatus basically must contain the arrangement of the apparatus in space and its function in time, if we are to be able to say anything at all about the phenomena... The argument is thus above all that the measuring instruments, if they are to serve as such. cannot be included in the actual range of applicability of quantum mechanics" $[108$, p. 313].

So classical terms must be retained, but their use will be limited by the considerations arising from the quantum postulate. As Bohr stressed in a letter to Darwin [79, p. 89], "every notion, or rather every word, we use is based on the idea of continuity, and becomes ambiguous as soon as this idea fails". The classical framework must be revised so that ordinary language may still be used to provide unambiguous communication [79, p. 16].

Consistency may be attained by restricting use of particular evidence obtained about the system to circumstances where experimental conditions are provided to obtain that evidence. Different experimental conditions may provide different evidence but, "evidence obtained under different experimental conditions cannot be comprehended within a single picture, but must be regarded as complementary in the sense that only the totality of the phenomena exhausts the possible information about the objects" [18, p. 210].

In what Murdoch $[75, \mathrm{p} .59$ ] says comes as close to a definition of complementarity as Bohr ever gave, he says that the quantum postulate "forces us to adopt a new mode of description designed as complementary in the sense that any given application of classical concepts precludes the simultaneous use of other classical concepts which in a different connection are equally necessary for the elucidation of the phenomena" $[5$, p. 10]. Murdoch stresses that mutual exclusiveness and joint completion are both necessary for a complementary description of a system. We may obtain experimentally, or discuss theoretically, a value for any property of the system we choose, but such experimentation or such discussion automatically precludes determination or discussion of another property. Joint completion implies that the individual descriptions "together provide a complete description" [75, p. 61], as stressed by Bohr in the last words of the previous paragraph. 
Bohr calls the linking together of observed system and measuring apparatus wholeness: "the essential wholeness of a proper quantum phenomenon finds indeed logical expression in the circumstance that any attempt at its well-defined subdivision would require a change in the experimental arrangement incompatible with the appearance of the phenomenon itself" [109, p. 72]. Bell [101] comments that, in quantum experiments "the results have to be regarded as the joint product of 'system' and 'apparatus', the complete experimental set-up", adding "as we learnt especially from Bohr".

Again it should be stressed how much of Bohr's analysis is linguistic, or semantic [75, p. 145] (though, as noted in section 3.3.1, some would prefer to say pragmatic). If a system has a value for variable $A$, the argument is not so much that complementary variable $B$ has no value; rather, in the circumstances in which a measurement of $A$ may be made, the concept of $B$ is inapplicable [18, p. 211]. Heisenberg perhaps goes further in his views on language than Bohr. In a letter to Stapp [88], he writes, "it may be a point in the Copenhagen interpretation that its language has a certain degree of vagueness, and I doubt whether it can become clearer by trying to avoid the vagueness".

The central example of complementarity, as stressed by Bohr at Como, is what Murdoch calls kinematic-dynamic complementarity. A measurement of a spatial coordinate requires a completely different experimental arrangement from a measurement of a component of momentum. Furthermore, in his discussions with Einstein [18, p. 212], Bohr showed that the circumstances in which a precise measurement of position may be made are precisely those in which an indefinitely large uncontrollable transfer of momentum between observed and observing systems may take place. Similarly, in an experiment to measure momentum exactly, one totally loses the possibility of locating the particle in space. Knowledge of spatial and momentum coordinates would provide complete knowledge of the system classically. Both could be available simultaneously in the classical theory, but not according to quantum theory. So position and momentum, or kinetic and dynamic variables, or, as Bohr put it [78], space-time coordination and the claim of causality (that is to say, the laws of conservation of momentum and energy) are complementary features of the situation.

These types of argument are, of course, analogous to those used by Heisenberg [80] in his demonstration of the indeterminacy relations, particularly his $\gamma$-ray microscope. The connections between Bohr's framework of complementarity, and Heisenberg's indeterminacy principle, both historical [79, pp. 90-97] and logical [79, pp. 127-141] are of great interest. Here we mention only that the common textbook idea that the two are practically synonymous is unfortunate; complementarity is a general position that extends far beyond the scope of any individual example, however important, and, at least in Bohr's eyes, far beyond physics [79, ch. 6].

Also in his Como article, Bohr discusses another example of complementarity - that between wave and particle models of light, and also matter. These models provide "different attempts at an interpretation of experimental evidence in which the limitation of the classical concepts is expressed in complementary ways"; we are dealing with "not ... contradications, but with complementary pictures of the phenomena, which together offer a natural generalisation of the classical mode of description".

While these quotations certainly sanction the idea of wave-particle complementarity, both Murdoch [79, p. 79] and Folse [75, p. 23] believe that it should be considered in a secondary position to kinematic-dynamic complementarity. As Folse says, neither wave nor particle model seems without question necessary for description of atoms and light, whereas Bohr's argument is that position and momentum coordinates are classical coordinates necessary for description, even though mutually exclusive. Neither does it seem clear that wave and particle pictures together form in any sense a complete description. Indeed it seems by no means obvious that a third picture could not be discovered which would, on its own, represent atomic systems in a satisfactory fashion. Murdoch [79, p. 66] argues that, while it may be attractive on occasion to identify each of wave and particle models with one or 
other of position or momentum measurement operations, such identification is certainly not universal. and is therefore probably misleading.

We will discuss two further points about Bohr's ideas, first his argument that measuring instruments cannot be included in the range of applicability of quantum mechanics. As Jammer [4, p. 473] points out, though, at the same time, measuring instruments were subject to the indeterminacy relations, and the analysis of many of Bohr's famous thought-experiments with which he argued against Einstein depended precisely on this factor. Jammer says, "the double nature of the macroscopic apparatus (on the one hand a classical object and on the other hand obeying quantum mechanical laws) . . remained a somewhat questionable or at least obscure feature in Bohr's conception of quantum mechanical measurement".

Bohr's views on the measuring device (contrasting strongly with von Neumann's willingness to write down a state vector for such a device) put a severe limitation on the kind of situation that quantum theory may deal with. As Stapp [88] says, "In the Copenhagen interpretation the notion of an absolute wave function representing the world itself is unequivocally rejected. Wave functions . . are associated with the studies by scientists of finite systems. The devices that prepare and later examine such systems are regarded as parts of the ordinary classical world. Their space-time dispositions are interpreted by the scientist as information about the prepared and examined systems. Only the latter systems are represented by wave functions."

The fact that - again unlike von Neumann-Bohr considers measurement a purely physical interaction [79, p. 201] should render him immune to any charge of subjectivity. For von Neumann, a macroscopic measuring device may be part of a chain between observed object and irredeemably subjective "abstract ego"; for Bohr, the first macroscopic object in such a chain marks the place where measurement occurs. Talk in Bohr's early work of the "observer" may thus mislead. Such a term is only a rather unfortunate synonym for a measuring device; there is no "mental-physical interaction", and consciousness plays no role $[79$, p. 24].

The second point to be discussed is Bohr's response [110] to the EPR argument [8]. As is well-known, the argument studied two systems that had interacted during a certain period, but then separated. The state vector remains entangled; it is not a product of a state vector for each system, but a sum of such products. A measurement of either $x$ or $p$ may be made on the first system. Because of the entangled (nonfactorisable) nature of the state vector, a measurement of $x$ on the first system, $x_{1}$, will yield a value of $x_{2}$ also; similarly a measurement of $p_{1}$ yields a value of $p_{2}$ as well. But, assuming some basic ideas on locality, neither measurement would have disturbed the second system, which must therefore have had elements of reality corresponding to these values of $x_{2}$ and $p_{2}$ in the absence of any measurement on either system. Such a situation is in conflict with conventional quantum theory, which EPR, therefore, declares to be incomplete.

Bohr's reply follows very closely the analysis of this section. He admits that "of course there is in a case like that ... no question of a mechanical disturbance of the situation under investigation [system 2]". However, the basic principle of complementarity tells us that, if we wish to undertake or even consider a measurement $x_{1}$, we must eschew any discussion of the value of $p_{1}$. A measurement of $x_{1}$ will allow us to deduce a value for $x_{2}$; contemplation of such a measurement will allow us to contemplate such a value. But either measurement of $x_{1}$, or even contemplation of such a measurement, prohibits any contemplation of values for $p_{1}$ or $p_{2}$, and this is sufficient to refute the EPR argument. As Bohr puts it, there remains "the question of an influence on the very conditions which define the possible types of predictions regarding the future behaviour of [system 2]". Murdoch [75, p. 175] says, "Bohr . . does not think that this sort of dependence involves any causal interaction between the objects in question, 
any sort of action at a distance, rather, what we do at one object may create a physical context which determines what we can meaningfully say about some other, spatially separated object."

One way of summarising Bohr's reaction to the EPR paper could be to say that the completeness of quantum mechanical description, challenged by EPR, was saved according to Bohr's view by the feature of "wholeness". Bohr's tenet was essentially that a particle, even after having ceased to interact with its partner, cannot be considered to be an independent seat of "physically real" attributes; or, in other words, dynamical independence does not necessarily mean kinematic independence as well. This holistic aspect was in Bohr's view unanalysable even in principle. It may be interesting to remark here that Aristotle's insistence in De Partibus Animalium about the priority of the whole over the parts, instead of treating the whole merely as the sum of its parts for the purpose of scientific investigation, can be regarded as an early analogue to Bohr's position.

\subsubsection{A few commentators on Bohr}

There has been a great deal of discussion of Bohr's point of view, and here we restrict ourselves to a very few commentators whose remarks are either of very general importance, or summarise widely held views, or are specially relevant for the subject of this paper.

As was stated in section 1 , the 1950 s were a testing time for the Copenhagen interpretation. By the end of the decade, a range of different opinions had been established, and a good example of this is seen in two important reviews published at this time, by Hanson [111], and by Feyerabend [87].

Hanson's article strongly supported Copenhagen. While he does not specifically rule out the right to challenge this orthodoxy, his paper is a strong rebuttal of those who had, in fact, questioned complementarity, which, Hanson claims, is not just a "philosophical afterthought", but "figured essentially" as "basic to every operation with the notation" in what Hanson describes as "the greatest contribution to physical theory of our time", Dirac's theory of the electron [112]. The Copenhagen interpretation has proved itself "to work in theory and practice"; if you doubt it, you are requested to "ask your nearest synchrotron operator".

Hanson is scathing towards "yesterday's great men" (among whom he includes Einstein, Schrödinger, von Laue and de Broglie). Their "amorphous sighs", he suggests "have not offered one scrap of algebra to back up their grandfatherly advice". He is possibly even more critical of the proponents of hidden variables, in particular Bohm. Each of their formalisms, he points out, "ultimately destroys the symmetry properties which have been the power and glory of quantum theory". Bohm, he says, admits that "his reinterpretation affected no known facts, but only added extra philosophical notions of heuristic value", and Hanson adds, "Bohr et Heisenberg n'ont pas besoin de cette hypothese".

Hanson does not claim "that philosophers ought to discontinue all attempts to develop proposals which counter the Copenhagen interpretation". He adds though that "until . . a new interpretation ... works in every particular as well as does the old one" such "efforts" should be called "by their proper name, "speculations"". Admittedly "this makes them no less worthwhile".

Despite this last remark, it is difficult to sum up Hanson's approach as other than totally complacent. For him, all the successes of quantum theory accrued to the Copenhagen interpretation. We are not in a genuine way "free to invent and to consider other metaphysical interpretations", because such a proposal "obscures the historical, conceptual and operationally successful role of the Bohr view ... as opposed to other interpretations".

Feyerabend's views, at least in retrospect, appear much more open-minded and forward-looking than those of Hanson. Feyerabend is by no means an opponent of Copenhagen, being "prepared to defend [it] as a physical hypothesis" and "to admit that it is superior to a host of alternative interpretations" 
[87, p. 201]. Indeed much of his paper is devoted to showing its "superiority"; Feyerabend claims to have "tried to exhibit the advantages of Bohr's point of view", and to have "defended this point of view against irrelevant attacks" [87, p. 231].

But Feyerabend specifically rules out any suggestion that the framework of complementarity is established permanently and is not open to challenge. He strongly disagrees with Rosenfeld's view [62, p. 41] that "any new conception which we need will be obtained by a rational extension of the quantum theory" (preserving the indeterminacies), and that "new theories of the microcosm will therefore be increasingly indeterministic". Feyerabend regards this "dogmatic philosophical attitude with respect to fundamentals" to be "fairly widespread", "completely unfounded" and "a very unfortunate feature of contemporary science" [87, p. 231]. "Now as ever", he replies, "we are free to consider whatever theories we want when attempting to explain the structure of matter".

This involves Feyerabend in a direct confrontation with Bohr's views on the a priori nature of classical concepts. Such are, in Bohr's eyes, necessary for reporting experience, and alternatives are, in any case, beyond the scope of the human power of invention. Feyerabend attempts [87, p. 230] to show that just such arguments (perhaps even stronger ones) could have been used to suggest that Aristotelian physics could not be superseded. Feyerabend believes that Bohr's arguments on classical concepts are unsound, though he suggests $[87$, p. 201] that the issue concerning the foundations of quantum theory "cannot be solved by alternative interpretations of the present theory", only by "the construction of a new theory".

Feyerabend is prepared, therefore, to be far more tolerant than Hanson towards speculations which may take a considerable period to challenge the established orthodoxy across the board. Thus he is interested in the "unpopular and fairly general speculations" [87, p. 232] of Bohm. (As early as 1960, incidentally, Feyerabend is prepared to dismiss as irrelevant von Neumann's arguments against hidden variables.)

Some specific points in Feyerabend's analysis are also worth reporting. First. in discussing the quantum postulate, and its effect on interactions between systems $\mathrm{A}$ and $\mathrm{B}$, he insists that the reasons why it is meaningless to ascribe intermediate states to A and B are not philosophical (positivistic) but physical. (We recall Feyerabend's description of complementarity as a "physical hypothesis".) "The proposed solution", he says [87, p. 197] "does not contain any reference to knowledge or observability. It is not asserted that during the time of transfer A and B may be in some state which is unknown to us, or which cannot be observed. For the quantum postulate does not merely exclude the knowledge or the observability of the intermediate states; it excludes the intermediate states themselves. Nor must the argument be read as asserting, as is implied in many presentations by physicists, that the intermediate states do not exist because they cannot be observed. For it refers to a postulate (the quantum postulate) which deals with existence, and not with observability. The emphasis upon the existence of predictabili$t y$ is not satisfied either. For this way of speaking would again suggest that we could predict better if we only knew more about the things that exist in the universe, whereas Bohr's suggestion denies that there are such things whose detection would make our knowledge more definite." Feyerabend suggests that the way in which terms become "inapplicable" in the complementarity argument may be analogous to a physical term like "scratchability" becoming inapplicable when a body starts melting.

Secondly, Feyerabend discusses the way in which he claims complementarity suffered "reformulation" as a result of the EPR argument. Up till now, we have spoken of the Copenhagen interpretation as static, or, at most, evolving gradually. According to Feyerabend [87, p. 193], it has only been possible for this impression to be given because of the "vagueness of the main principles of the creed" which "allows the defendants to take care of objections by development rather than by reformulation, a 
procedure which will of course create the impression that the correct answer has been there all the time and that it was overlooked by the critic". The attitude of Bohr and his followers, according to Feyerabend, "has very often been one of people who have the task to clear up the misunderstandings of opponents rather than to admit their own mistakes".

While the hidden-variables question is one example of this according to Feyerabend, the most important is, indeed, Bohr's response to EPR. Feyerabend calls this [87, p. 217] acknowledgement of the "relational character" of the quantum mechanical state. State descriptions are "relations between the systems and measuring devices in action, and are therefore dependent upon the existence of other systems suitable for carrying out the measurement". In EPR "while a property cannot be changed except by interference with the system that possessed the property, a relation can be changed without such interference". For example, the state "being longer than $b$ " of a rubber band may change if we interfere with the rubber band, or, if, without disturbing the rubber band, we change $b$.

Feyerabend says, "Bohr's suggestion proves ... extremely useful" [87, p. 218], but insists that it "refutes the earlier picture where a measurement glues together, with the help of an indivisible quantum of action, two different entities, viz., the apparatus on the one hand and the investigated object on the other". (In contrast to the relational point of view, Feyerabend suggests [87, p. 219] that, until the time of EPR, Bohr's view was that the dependence of a microsystem's behaviour on the experimental arrangement was due to a genuine interaction; he likens this to attributing the difference in the properties of an object observed in a moving frame of reference to an interaction with the frame.)

Lastly we mention that Feyerabend makes several pertinent comments concerning the ensemble interpretation; these comments will be discussed in sections 5 .

Thirty years after the Hanson-Feyerabend debate, it is clear that Feyerabend's views prevailed; indeed they probably underestimated the pace of changes of attitude. Today many views alternative to those of Bohr have been presented, and some are given wide and continuing attention. Bohm's theory in particular, rejected by Hanson as irrelevant and unprogressive, is now thought by many to require serious consideration. (It is often now called the de Broglie-Bohm theory, since Bohm's work was along lines originally developed, but subsequently rejected, by de Broglie [113]; or, as Bohm prefers, the "quantum potential" approach [114].) Bell [101] considers that, far from being conceptually insignificant, this theory shows that "vagueness, subjectivity and indeterminism are not forced on us by experimental facts, but by deliberate theoretical choice" (though he admits that even such opponents of Copenhagen as de Broglie and Bohm themselves, and Einstein, did not actually like it very much). Bohm's theory was one of the main sources of inspiration for Bell's own important work [20,21].

So far has the pendulum swung that, while most working physicists would still probably describe themselves as following the Copenhagen line, few discuss Bohr's own work. Among leading workers in the area, Bell [22] admits that he "never got the hang of complementarity" [22, p. 194], and regards it as one of the "romantic" world views inspired by quantum theory, as opposed to the "unromantic 'professional' alternatives" he supports. On the EPR problem, he reports on having "very little understanding of the position of ... Bohr" [22, p.155].

Squires [115, p. 118] says that the Copenhagen interpretation "is very vague and answers few of the questions; anybody who thinks about the subject today would be unlikely to find it satisfactory". He also [116, p. 180] says, "the orthodox interpretation begins to sound like 'mumbo-jumbo', a collection of empty words that indicate only that we really do not understand quantum theory", and quotes Gell-Mann [117] to the effect that "The fact that an adequate philosophical presentation has been so long delayed is no doubt caused by the fact that Niels Bohr brainwashed a whole generation of theorists into thinking that the job was done fifty years ago". 
Perhaps the pendulum has swung too far, and it is interesting that it is philosophers, Murdoch [75] and Folse [79] who are stimulating renewed study of Bohr and his ideas. In his book, Murdoch makes some general observations on Bohr's beliefs that go considerably beyond the scope of our previous discussion, and uses them to produce an axiomatised version of Bohr's theory of measurement, to be compared, to a considerable extent, with that of von Neumann. These ideas are particularly relevant for discussion of ensemble interpretations. Murdoch recognises [75, p. 107] that his views may be rejected, not just by those hostile to Bohr's approach, but by some of those sympathetic to Bohr, and, in sketching Murdoch's ideas, we take the opportunity of summarising how such a critic might reply to them.

In section 3.3.2, we discussed Murdoch's starting point, which is that, in quantum physics, a measurement gives us the value the quantity being measured possessed before the measurement.

Murdoch [75, p. 125] represents a nonideal measurement by $\psi_{i} \phi_{0} \rightarrow \xi \phi_{k}$, where the object is initially in an eigenstate $\psi_{i}$ of the observable $A$ being measured, and finally in state $\xi . \phi_{0}$ and $\phi_{k}$ are initial and final states of the instrument observable. In general, Murdoch says " $\xi$ is not equal to $\psi_{k}$ [as would be the case for a measurement of the first kind] or even orthogonal to it; $\xi$, moreover, may not be the value of $A$ immediately before the measurement", so that $\phi_{k}$ should presumably be correlated with $\psi_{i}$.

As stated above, Murdoch bases his argument on Bohr's stated and well-known view that "the observation problem of quantum physics in no way differs from the classical physics approach" [118, $p$. 3]. Murdoch suggests [75, p. 111], "if the value observed were produced by the interaction between the object and the instrument, then it would be quite inappropriate to speak of a measurement taking place."

To what extent does this reflect Bohr's views? The sceptic may suggest that when Bohr talks about measurement as a classical process, he is referring to the provision of a suitable apparatus, and the way in which the results will be described: "The argument is simply that by the word 'experiment' we refer to a situation where we can tell others what we have done and what we have learnt and that, therefore, the account of the experimental arrangement and of the results of the observations must be expressed in unambiguous language with suitable application of the terminology of classical physics."

Murdoch's other quotation from Bohr [110, p. 73], his statement that terminology such as "creation of physical attributes to atomic objects by measurements" is "apt to cause confusion" because the words "attributes" and "measurements" are used in a way incompatible with common language and practical definition, may appear more difficult for the sceptic to argue around (particularly the reference to "attributes"). Probably the sceptic's response must be that the whole concept of complementarity is designed to limit the scope of physical discussion to those circumstances where classical terminology may be used in an unambiguous fashion. Since Murdoch is going outside this area of discussion in contemplating values of quantities before measurement, it may be thought inconsistent of him to utilise Bohr's remarks.

Let us for the moment, though, accept his idea and follow its consequences. His next problem must be that, according to most adherents to Copenhagen, the quantity being measured will not, in general, have a value before the actual measurement. (In the nonideal measurement above, $\psi_{i}$, they would say, in general, does not correspond to an eigenstate of $A$.) At first sight, it appears that Murdoch is assuming what he calls an intrinsic-values theory of properties, according to which, all observables relating to an object have, at any moment, definite values. Thus the state vector does not contain all information available about the system it describes (so, in fact, Murdoch's assumption that the initial state of $A$ is an eigenstate would be unnecessary) and this theory is equivalent to what we introduced as a PIV ensemble interpretation at the very beginning of this review.

Murdoch, however definitely rejects this theory [75, p. 107] and says that Bohr did the same. Rather 
he holds what he calls the objective-values theory of measurement, and specifically states that when Bohr said the observational problem of quantum physics is the same as the classical approach, "what he has in mind is the objective-values theory" [75, p. 107].

This ingenious theory says that a measured observable does have a pre-existing value, which the measurement then reveals. However [75, p. 150], "the theory does not state, or even entail, that an observable has a pre-existing value whether or not it is measured", as would the intrinsic-values theory. Thus "Bohr can consistently say that successful measurement of an observable reveals its pre-existing value, and also that talk of a pre-existing value is meaningful only in a context in which the measurement has been made. He is, of course, required to deny that we can meaningfully say that the pre-existing value that is revealed by measurement would have been present if no measurement had been made."

Murdoch argues [75, pp. 128-133] that many of the well-known difficulties of the intrinsic-values theory, which will be discussed in section 5 , do not apply to the objective-values theory.

The choice between intrinsic-values theory and objective-values theory is intimately linked with another choice concerning the argument of complementarity - between what Murdoch calls [75, p. 148] the strong meaning condition and the weak meaning condition. Under the former, in order that an observable may be said to have a certain value, a measurement of that observable is required. Under the latter, the less severe restriction that a measuring apparatus appropriate for measuring the observable is in place is sufficient that the observable may be ascribed a value.

It appears easy to combine Murdoch's idea that measurement reveals a pre-existing value, the weak meaning conditiun, and the objective-values theory (but to reject the intrinsic-values theory). A measuring apparatus suitable for measuring observable $O$ may be put in place; under these circumstances $O$ may be ascribed a value, which the measurement then reveals. On the other hand, if one adopts the strong meaning condition (but retains Murdoch's view above of measurement), one would appear forced to the view that all observables have values at all times, the intrinsic-values theory. Murdoch is, however, obliged to admit [75, p. 148] that Bohr adopts the strong meaning condition, but regards it as a mistake; "he ought", Murdoch believes [75, p. 154] "to have adopted the weak meaning condition" (in order that he could hold the objective-values theory).

Also connected with the question of objective-values and intrinsic-values theories is the question of interpretations. As Murdoch says [75, p. 150]: "If Bohr adopted the ontic interpretation, then indeed, he could not consistently have held the objective-values theory, since on this ontic interpretation, an observable does not have a definite value immediately before it is measured". Murdoch does not, however, believe that Bohr did adopt the ontic interpretation, as already discussed in section 3.1. These views of Murdoch then lead directly on to those in the following sections of this paper.

We have described Murdoch's formalisation of Bohr's views as ingenious. It enables one of the central planks of complementarity (in one particular version, the weak meaning condition), to be coupled to an approach to measurement less vague than that usually attributed to Bohr, yet less restrictive than the intrinsic-values theory. Yet sceptics will ask: is it not entirely fanciful that mere setting-up of an apparatus can "create" a value for an observable? (Does it really do less damage to ordinary language than that measurement itself creates the values, a form of words derided by both Bohr and Murdoch [75, p. 105]?)

Murdoch argues [75, p. 145] that the point is not so much the ontic one-does $O$ have a value? Rather it is the semantic one - may we avoid ambiguity in referring to the concept of $O$ ? The critic may reply, though, that it is Murdoch himself who has moved from "semantic" to "ontic" with the objective-values theory.

Those who agree that Murdoch does not provide an entirely satisfactory formalisation of Bohr's 
theory of measurement, may still differ on the more fundamental point. Is it because Bohr's ideas themselves are too subtle and high-flown to be represented in this perhaps rather crude way, or is it that Murdoch exposes the lack of real substance in the Bohrian position, usually disguised by obscuring prose?

\subsection{Summary}

In section 3, a range of rather varying positions has been sketched. They have been discussed in some detail to show that the "orthodox" position has greater variety and depth than is sometimes suggested by the advocates of ensembles. All have difficulties - von Neumann's "observer" required to collapse wave functions, Bohr's insistence on the a priori nature of classical physics, particularly for measurement.

There are also basic questions at a more fundamental level than that of measurement. One might inquire about the underlying meaning of the wave-function concept, or request a physical explanation of superposition. How can a superposition of states with $S_{z}=+1 / 2$ and $S_{z}=-1 / 2$ represent an eigenstate of $S_{x}$, or a superposition of $\mathrm{K}^{0}$ and $\overline{\mathrm{K}}^{0}$ mesons be a different particle altogether, the $\mathrm{K}_{\mathrm{S}}^{0}$ or $\mathrm{K}_{\mathrm{L}}^{0}[119]$ ? Von Neumann's theory is basically mathematical and cannot even respond to such questions, and the only response from Bohr's more conceptual approach is that no answer can be given.

Are these problems a result of the immutable limitations of human beings in action and comprehension? Or should they be regarded as constituting an admitted blemish, but a rather esoteric one, remote from many more important concerns, and well worth leaving on one side? Or, as Ballentine and his supporters would maintain, are they such as to demand that this category of interpretations be totally rejected? And if so, does Ballentine's own proposed solution of an ensemble interpretation fare any better? These questions, especially the last one, are the theme for the remaining sections of this paper.

\section{Ensemble interpretations; a general survey}

\subsection{Types of ensemble interpretation}

From the previous sections, it will be clear that we have met two motivations for the introduction of ensemble interpretations. First, from section 2, supporters of relative-frequency interpretations of probability are almost certain to hold that any probabilistic theory, such as quantum theory, must be a theory of ensembles. Newton [120], for example, writes, "the most pernicious idea concerning probability is that it is an intrinsic concept about an individual... [W] hen we give a probability, the class or ensemble, from which the object is to be drawn, must be specified by its properties". He goes on to say that "the state of a system" in quantum mechanics . . is (the symbolic representation of) "the ensemble to which it belongs".

The second motivation is to add structure or "support" to quantum theory, thus explaining or removing its indeterministic nature, or reducing it to a realistic, though perhaps probabilistic form.

A great variety of approaches is included here, and, traditionally and conveniently, different names are used for different types. For instance, the term "hidden variables" is usually used for attempts to provide fairly "minor modifications" $[4$, p. 440] to the usual theory. Attempts to make such terminology watertight are abortive, as demonstrated by a dispute between Bell and Jammer. Bell began his famous 
paper on EPR [21] by invoking the name of Einstein in support of hidden variables. Jammer [4, p. 254] criticised this comment, saying that Einstein never endorsed any hidden-variables theory, hoping, rather, that a unified field theory would solve the quantum difficulties. (See section 4.3 for further comment.) Bell, however [22, p. 90] asserted that Einstein's belief in classical field theory, implying the use of continuous functions in four-dimensional space-time, in no way excludes belief in hidden variables. According to Bell, such continuous functions can be viewed as a "particular conception" of hidden variables.

Similarly, Jammer excludes from his chapter titled "Statistical Interpretation" [4, ch. 10] what he calls "Stochastic Interpretations" [4, ch. 9], the objective of which he describes [4, p. 418] as being "to show that quantum theory is fundamentally a classical theory of probabilistic or stochastic processes". While the distinction is useful, it cannot be drawn rigorously. De la Peña and Cetto, for example, end their paper [121] titled "Stochastic theory for classical and quantum mechanical systems" by saying, "The theory of QM developed here is in full agreement with the so-called statistical interpretation of QM supported by Einstein . . . and still unpopular among physicists; it is a hidden-variables theory." In particular they refer to Ballentine's paper [3].

Strictly, then, our title of "ensemble interpretations" should include the large number of theories usually called "hidden-variables" or "stochastic". In fact, we largely exclude (with one crucial exception mentioned below) "hidden-variables" theories; these have been reviewed authoritatively by Belinfante [122]. "Stochastic" interpretations have also been recently reviewed authoritatively by Gudder [123], but in this case it is felt worthwhile to sketch, in section 4.6, a few recent approaches, particularly those illuminating the more central themes of this review.

The exception to our policy on hidden-variables theories is what we [1] have called (and will call here) the PIV (pre-assigned initial values) ensemble interpretation; it is Murdoch's intrinsic-values theory [75] (section 3.3.3). Fine [124] has called it the complete-value thesis, and elsewhere [125, p. 43] a random-values representation. Guy and Deltete [126] speak of the Gibbs ensemble, and this terminology makes the point that it is just the natural assumption borrowed from classical theory that, at all times, all variables have values, values which are then available to be discovered in measurements. In an ensemble of spin-1/2, particles in an eigenstate of $S_{z}$ with eigenvalue $+1 / 2$, for example, all the particles would have $S_{z}=1 / 2$, but half would have $S_{x}=1 / 2$, half $S_{x}=-1 / 2$, and so on.

So natural and pleasing is this interpretation that, for many, it just is the ensemble interpretation (for example, Popper [65, p. 7]). Indeed, Fine is prepared [125, p. 43] to call it exactly that, using the term "statistical interpretation" for the more general concept. It goes without saying, though, that any orthodox view is rigidly opposed to such a position, imagining it to have been disposed of over sixty years ago. Some of the arguments have been rehearsed in section 3 , and they will be returned to in section 5. In section 4.5, though, a number of recent theories in this general area are discussed, following sections 4.3 and 4.4 where the views of Einstein and Ballentine are examined, and section 4.2 where there is a brief discussion of "completeness".

It is worth mentioning that some of those who use a PIV interpretation, either more or less explicitly, may not think of it as a hidden-variables theory. D'Espagnat [127] says, "Some authors [d'Espagnat cites our ref. [3]] mention an 'ensemble theory' in which it is assumed that a particle has at a given time both a definite position and definite velocity and yet claim that this theory (which is supposed to reproduce exactly the usual quantum-mechanical results) is compatible with the assumption that no hidden variables exist. It must be conjectured that these authors understand the expression 'hidden-variable hypothesis' in some global sense, different from what the words themselves seem to suggest." 
To conclude this section, we return to the two motivations for ensemble interpretations mentioned at the beginning. Both are clear-cut and beyond criticism. In the argument from definition of probability, there is no initial aim to solve the difficulties of quantum theory, though such success may be claimed to result. Solving these difficulties may or may not be the chief aim of structured ensemble theories (hidden variables, stochastic) but these theories should be judged on their merits.

A third motivation for supporting an ensemble interpretation seems to be merely to avoid the difficulties and "paradoxes". Of course, there is nothing wrong with that as such, but it may lead to rather crude distortions of the "orthodox" position. For example, in supporting an ensemble interpretation, (1) Tschudi [128] criticises the "erroneous dogma" that the wave function is "a property of the physical object concretely present"; (2) Ballentine [129] says, "the quantum state describes not an individual system, but an ensemble"; (3) Zweifel [130] rejects "the quantum mechanical description of individual events"; (4) Roychoudhuri [131] argues, "quantum measurements never refer literally to an isolated single particle"; and (5) Ballentine [3, p. 361] says, "in general, quantum theory predicts nothing which is relevant to a single measurement".

Detailed exegesis of these statements is risky because of the vagueness of such phrases as "property", "describe", "refer to", and "is relevent to". Taking this risk, however, we would suggest that (1) is a gross caricature of even the most crude orthodox interpretation; (2) is rather misleading unless made more technical by adding the word "completely", when a proper discussion may be entered into, as in section 4.2. On (3) and (4) (and taking an "event" to be a measurement) we would suggest that quantum mechanics may describe the result of a measurement, although it cannot, of course, predict it as it is a probabilistic theory; a measurement is (or may be) on a single particle. On (5), the probabilities predicted by quantum theory are surely "relevant".

One feels that these proponents of ensembles are claiming too easy a victory over orthodox interpretations. They are either damning these interpretations for saying what they do not say, or, in effect, dismissing them because they do not (cannot) say things they do not claim to say and there is no requirement for them to say. This type of comment often amounts to little more than saying that, because quantum theory uses probabilistic ideas, it is inherently an ensemble theory, which is true if. but only if one restricts oneself to a frequency interpretation of probability.

Much the same goes for Blokhintsev's suggestion [17, p. 50], quoted with approval by Ballentine [3. p. 379], that since "it is stated in many textbooks of quantum mechanics that the wavefunction is a characteristic of the state of a single particle . . it would be of interest to perform such a measurement on a single particle... which would allow us to determine its own individual wave function. No such measurement is possible". In fact, there just seems no compelling reason why such a measurement is called for.

Actually Penrose [132, p. 105] has given what is, in many ways, an answer to the spirit of Blokhintsev's challenge. If the wavefunction of the system is $\psi$, let us form the Hermitian operator, $Q$, equal to $|\psi\rangle\langle\psi|$. According to von Neumann [14], any Hermitian operator, in principle, may be made the subject of a measurement, so we may measure the observable defined by $Q$. Apart from phase factors, the state $|\psi\rangle\langle\psi|$ is the only one for which a measurement of $Q$ certainly gives the answer unity, and Penrose suggests, therefore, that the property of being in state $\psi$ is entirely objective. Though, of course, such a measurement cannot determine any wave function, the argument does appear to demonstrate that a wave function is a "characteristic of a single particle". While Penrose's argument is interesting, it relies crucially on the postulate from von Neumann, itself much questioned. But, as already stated, we are not sure the original challenge of Blokhintsev has any real legitimacy. 


\subsection{Completeness}

Much of the preceding discussion may be sharpened by the notion of "completeness". To say, loosely, that a wave function "describes" a single system could be, for example, to say it gives a complete classical description, or a specific prediction of the result of any experiment that may be performed on it.

For definition of a "complete" description, we turn to the EPR paper [8], the title of which was, of course, "Can quantum-mechanical description of physical reality be considered complete?" For a theory to be complete "every element of the physical reality must have a counterpart in the theory". For us (unlike EPR) establishing "physical reality" is not a main issue. We may merely note that, if a particle "has" simultaneous exact values of $x$ and $p_{x}$, and since quantum theory cannot provide a counterpart for these values, quantum theory must be incomplete - a sketch of the EPR argument.

Before applying this idea to our case, it is worth studying the idea of completeness a little more thoroughly. For general background, Jammar [82, p. 129] gives an excellent account of the historical background of the concept, and its importance in the genesis of the EPR paper.

Fine makes things more complicated, but raises some important issues. In discussing Einstein's work $[125$, p. 70$]$, he finds three "interpretations" of the word "completeness". The first, which Einstein calls Bornian, asserts that any probabilistic theory is necessarily incomplete. Probabilities cannot be fundamental, but may only arise as averages over quantities which are fundamental. The second interpretation, which Einstein calls the Schrödinger conception, allows probabilities to be fundamental. A probabilistic theory may be complete if it "tells the whole truth about that state of affairs" $[125, \mathrm{p}$. 71]; otherwise it is incomplete. In Einstein's correspondence, Fine [125, p. 71] detects a third conception of completeness, which Fine terms "bijective completeness" - " $\psi$ must stand in a one-to-one correspondence with the real state of the real system". Fine [125, p. 72] complains that Einstein often "runs together Schrödinger incompleteness with the quite different idea of bijective incompleteness, taking arguments for the latter as establishing the former".

What is usually called completeness is probably the bijective conception (though, in practice, as already implied, there is considerable overlap with the so-called Schrödinger concept). The so-called Bornian concept is entirely different. (It should not be supposed, incidentally, that Einstein's use of the names of Born and Schrödinger, particularly the first, was really appropriate. His reasons are mentioned by Fine [125, pp. 77, 78]. Although Schrödinger was not a supporter of the Copenhagen position, his initial electromagnetic interpretation may have continued to attract him sufficiently to justify the use of his name. Born and Einstein had a substantial, and often mutually confusing, correspondence on the interpretation of quantum theory [133, pp. 163-228]; as Fine says, use of Born's name appears to confuse the general Born rule with Einstein's own ensemble concepts.)

Let us now apply these ideas to quantum theory. Ballentine [3, p. 360] distinguishes the orthodox interpretation, which asserts that "a pure state provides a complete and exhaustive description of an individual system" from the statistical, which "need not provide a complete description of an individual system".

With these definitions, (bijective) completeness tells us that, if structure of hidden-variable type actually "exists", orthodox interpretations are indeed incomplete, and, of course, hidden-variables theories are open to consideration. (We must remember that PIV-ensemble theories are of this type.) But Ballentine's definitions give no encouragement to consider ensemble interpretations without such 
structure ("minimal" ensemble interpretations $[2$, p. 76]), nor even for the view that such interpretations are "completely open with respect to hidden variables".

If one were to use the so-called Bornian version of completeness, the only difference would be that, since fundamentally probabilistic arguments are ruled out, hidden variables would be demanded, so quantum theory would a priori be incomplete. This seems, incidentally, to be the argument of Hemion [134]: "By its very nature, the theory of statistics deals with situations where the details are unknown; if all details were completely known, then one would have no need to bring in statistics in the first place. Thus, in a trivial sense, all statistical descriptions of reality must be incomplete."

\subsection{Einstein (Fine, Rosen)}

Einstein has always rightly been regarded as the standard-bearer of those opposed to the Copenhagen "bandwagon", and any who oppose the orthodox interpretation are naturally inclined to claim his support for their own particular views. Ballentine [135] assembles the evidence that Einstein supported an ensemble interpretation. Jammer [4, p. 440] concurs: "[A]ll through his life Einsten adhered to the statistical interpretation of the existing formalism." (See also p. 119 of ref. [4].)

In 1936, Einstein wrote [7]: "The $\psi$ function does not in any way describe a condition which could be that of a single system; it relates rather to many systems, to 'an ensemble of systems' in the sense of statistical mechanics." In connection with problems of EPR type, he wrote, "The co-ordination of the $\psi$ function to an ensemble of systems eliminates every difficulty." Very similar statements are to be found in Einstein's "Reply to criticisms" in the Schilpp volume [18, p. 663].

There remain, though, two important areas of discussion. First, it is important to consider what type of ensemble theory Einstein supported. Secondly, as Ballentine and Jammer do not fail to point out, Einstein's advocacy of ensemble interpretations was not the whole story. Fresh light has been shone on these matters recently by Fine [125], who has studied the Einstein archive in depth, and then in a controversy between Guy and Deltete [126], and Fine himself [124].

To attempt to answer the first question, we may start by considering Einstein's advocacy of an ensemble interpretation at the Fifth Solvay Conference of 1927 [93; 4, p. 115; 135]. He postulated a simple thought experiment in which a particle impinges on a diaphragm, in the region surrounding a slit. A scintillation screen in the shape of a hemisphere is placed behind the slit. According to an orthodox interpretation (Einstein's viewpoint II), when a flash is detected at a definite point on the screen, a peculiar action-at-a-distance must have taken place. Before the flash, the particle is potentially present over the whole screen; at the time of the flash, it appears at one point, and not more than one. Einstein preferred viewpoint $I$ in which $\psi$ represents, not a single particle, but an ensemble distributed in space, and $|\psi(r)|^{2}$ represents the probability that a particle of the ensemble exists at $r$, so the action-at-adistance is not required.

In these brief comments, Einstein fathered the ensemble interpretation, and gave perhaps the first account and criticism of the wave function collapse. For our immediate purpose, the central point is that his ensemble interpretation was of PIV type; each particle has a definite, though unknown, position before it meets the screen.

Later, Einstein spoke, in the main, much less clearly about what type of ensemble model he supported. Fine [124, p. 968] writes: "So far as I have been able to discover . . Einstein nowhere offers a detailed account of the ... ensemble interpretation."

One presumes that Einstein made his ideas less rigid deliberately, perhaps because of the difficulties of PIV interpretations [125, pp. 43-50] (see section 5 here). However the PIV idea is still the most 
natural way to start thinking about Einstein's ensembles. Ballentine [135] writes that it was the idea of "an ensemble (in the Gibbian sense) . . that he was to adopt consistently in later years". Fine [124, p. 968] remarks: "Despite the lack of detailed textual material, many have taken Einstein's ideas on ensembles to amount to a local deterministic hidden-variables theory, or the equivalent statistical construct (as in [our] ref. [135])."

"To highlight the scarcity and vagueness of the textual data, and to challenge that conventional wisdom", Fine continues, "I offered a slightly different construct: namely, one in terms of my prism models." Fine had been developing these models for some years $[136 ; 137 ; 125$, pp. $51-53 ; 138 ; 124]$ in an effort to avoid the problems of other interpretations in reconciling the predictions of quantum theory with Bell's analysis [21]. Fine simply side-stepped Bells assumptions, by claiming that, of a given ensemble of systems, some are simply defective for having a particular measurement made on them. He says: "I do not mean simply that the particle will not [be passed]. I mean something stronger and stranger... [T] he particle simply will not show up at all; it could neither be detected as passing nor as not-passing that [analyser] (ref. [136], quoted in ref. [126]).

Fine continues $[124$, p. 985] that his approach parallels that which exploits the inefficiency of experimental tests [34] of the Bell inequalities. Pearle [139] and Clauser and Horne [140] noticed that, although the results for detected systems disagreed with the inequalities, creative use of the inefficiencies allowed local hidden-variables models, which were then produced by several authors [141-144].

Fine $[125$, p. 5] says, "it is not really my intention to make Einstein into a precursor of prism models", but he does produce quotations to build up a case that "the idea of a prism model ... does seem to fit Einstein's various remarks snugly" [125, p. 53], and concludes: "Overall ... I think the concept of a prism model fits the whole range of Einstein's ideas much more readily than does the concept of an ensemble interpretation [i.e., of PIV type]." [125, p. 56]. Guy and Deltete [126] criticise both the prism idea as such, and also the suggestion that Einstein's conception could resemble it. Fine's reply is ref. [124], from which we have already quoted.

Fine also shows here that Einstein never developed a consistent approach to the quantum theory. Sometimes, he says, Einstein [145] "describes the quantum theory in a perfectly orthodox, instrumental fashion ... according to which the quantum probabilities are probabilities for measurement outcomes and not for premeasurement values"; for good measure he suggests that, on occasion, Einstein [18, $\mathrm{p}$. 83] uses a much more subjective interpretation according to which $\psi$ expresses "what we know... about the system". And, of course, in some writings Einstein did use PIV language. Fine gives examples $[124$, p. 974], but perhaps the best one is really the EPR paper, where establishment of the simultaneous values of $x$ and $p_{x}$ is central to the argument. Yet significantly it is not the triumphant conclusion, to be developed further; that path had to wait for Bell nearly 30 years later. (See the mention of the Bell-Jammer dispute in section 4.1.) It is also very well-known that, in a letter to Born, Einstein dismissed the Bohm scheme [19] as "too cheap" [133, p. 92].

The reasons for Einstein's lack of interest in such theories, more generally his lack of interest in developing his ensemble ideas systematically, have been discussed in refs. [124-126]. For Einstein, ensembles were the only way to solve the internal problems of quantum theory, but they were "inadequate to the task of describing the real physical states of individuals" [126, p. 944]. ("Real states" are not necessarily to be identified with "classical state descriptions" [124, p. 974].) So developing his ensemble interpretation would have been a "waste of time" [126, p. 962]. Ensembles "provided no more than a setting rhetorically apt for calling attention to the incompleteness of the quantum theory" $[125, \mathrm{p}$. 5]. "Einstein chose his rhetoric cunningly. For who, learning that a theory is incomplete, could resist the idea that one ought to complete it?" [125, p. 58]. "Einstein is thus far less concerned to show what 
ensembles can do than he is to insist on what they cannot do" [126, p. 962]. "Einstein wanted his purely interpretative scheme to appear heuristically sterile" [125, p. 59].

Ensembles then demonstrate the incompleteness of quantum theory. For Einstein, though, making complete cannot be achieved by adjusting or augmenting the formalism as we have it ("from within", to use Fine's expression $[125$, p. 58]). He believes that "this theory offers no useful point of departure for future development" [18, p. 87], "just as . . one could not go from thermodynamics ( . . statistical mechanics) to the foundations of mechanics" [7]. And Einstein also rejects [18, p. 672] the view that one might just agree to leave the theory incomplete.

Rather, the theory must be completed "from without", by construction of a more general theory, encompassing general relativity [18, p. 81], and including the results of quantum theory as an approximation: "The statistical quantum theory would eventually take an approximately analogous position to the statistical mechanics within the framework of classical mechanics" [18, p. 672].

Einstein's general views are perhaps best expressed when he describes those of "contemporary physicists" from whom "[his] expectation departs most wildly". "They believe it is impossible to account for the essential aspects of quantum phenomena . . . by means of a theory which describes the real state of things by continuous functions of space for which differential equations are valid. They are also of the opinion that in this way one cannot understand the atomic structure of matter and of radiation... they believe that the apparently discontinuous character of elementary events can be described only by means of an essentially statistical theory." [18, p. 87].

Einstein's own view was the opposite, and so resulted his strenuous efforts in the last years of his life to develop a suitable field theory. In 1949 he reported his approach as follows. As compared to the theory of general relativity, the symmetrical tensor $g_{i k}$ is replaced by $s_{i k}+a_{i k}$, where $s_{i k}$ is symmetric, and $a_{i k}$ real or purely imaginary and antisymmetric. (Einstein notes that this extension seems natural, because the electromagnetic field has to do with an antisymmetric tensor.) A formula for curvature, and field equations may then be produced. Einstein comments: "The proof of their physical usefulness is a tremendously difficult task, inasmuch as mere approximation will not suffice", but he adds: "The theory here proposed . . . according to my point of view, represents a fair probability of being found valid, if the way to an exhaustive description of physical reality on the basis of the continuum turns out to be possible at all" [18, pp. 89-95].

He himself failed to make much progress, though Fine considers that he was "genuinely content to have made the effort" [125, p. 25]. As Ballentine [135] reports: "No nontrivial combination of general relativity with quantum theory has ever been accomplished. The unified field-theory program has not been able to encompass quantum phenomena, and the other extreme, 'quantization' of gravitation by forcing the equations of general relativity into the operator form of quantum mechanics, has also met severe difficulties."

We close this section by jumping to a brief comment about the views of Rosen expressed fifty years after EPR [82, p. 17]. While it would clearly be incorrect to identify Rosen's views in 1985 with those of Einstein either in 1935 or at any later time, it is interesting that Rosen's views on quantum theory appear to parallel Einstein's as presented here, though he appears to have lost faith in the idea of completion by a field theory. Rosen believes the ensemble theory explains the EPR problem, the ensembles being "coherent ensembles", as distinct from the "incoherent ensembles associated with mixed states in quantum mechanics, and those in classical physics." Rosen appears to be willing to use PIV notions when convenient, but, just like Einstein, not to wish to commit himself to any specific categorisation of the ensemble. 


\subsection{Ballentine (Popper)}

In this section, we report the main themes of the important paper by Ballentine [3]. Effectively this is to review the main elements of the ensemble theory. The name of Popper is included in the title because he has also been regarded as one of the main advocates of ensembles $[11 ; 65, \mathrm{p}$. 7]. However, he makes few arguments that are not included in Ballentine's paper, and we shall have few occasions to cite him directly.

In Ballentine's paper the following points are made or emerge:

(1) Type of interpretation. Does Ballentine consider "structured" or "unstructured" ensembles (in the sense of section 4.1 - "structure" implying hidden variables or similar)? His stated position is, "the Statistical Interpretation . . . is completely open with respect to hidden variables. It does not demand them, but it makes the search for them entirely reasonable." [3, p. 374]. Yet nearly all Ballentine's comments show that he is, in fact, contemplating a PIV ensemble, and thus, of course, hidden variables. For example, he states [3, p. 361], "a momentum eigenstate . . represents the ensemble whose members are single electrons each having the same momentum, but distributed uniformly over all positions". Also on p. 361 of ref. [3], he says, "the Statistical Interpretation considers a particle to always be at some position in space, each position being realised with relative frequency $|\psi(r)|^{2}$ in an ensemble of similarly prepared experiments". Later [3, p. 379] he states, "there is no conflict with quantum theory in thinking of a particle as having definite (but, in general, unknown) values of both position and momentum". Slightly less explicit is his statement [3, p. 361]: "Physical systems which have been subjected to the same state preparation will be similar in some of their properties, but not in all of them".

In his discussion of the Bohm [146] adaptation of EPR, Ballentine says [3, p. 363] that "[to deny] the reality of both $\sigma_{1 z}$ and $\sigma_{2 z}$ until the measurement has actually been performed" represents "an extreme positivist philosophy" and "entails the unreasonable, essentially solipsist position that the reality of particle 2 depends upon some measurement which is not connected to it by any physical interaction". Other related comments of Ballentine will be referred to in our own remarks on measurement and the uncertainty relations below.

Practically the only place in Ballentine's article where he appears to question the PIV idea is where, having said [3, p. 372] that "quantum theory is not inconsistent with the supposition that a particle has at any instant both a definite position and a definite momentum", he adds that "we are not compelled to accept or reject this supposition".

This point has been stressed, because, although philosophically minded readers have no difficulty in recognising it $[127 ; 2$, p. $77 ; 124]$, it is so foreign to physicists' expectations that they are inclined to miss it. We would suggest that it is unfortunate that in the most-quoted accounts of ensemble theories, studied in this section and the previous one, the PIV idea is not fully acknowledged, yet it is implicit in the background and so may be used practically inadvertently. It must be admitted that some authors are more explicit; Popper [65, p. 28] simply states, "we must ascribe to particles a precise position and momentum".

(2) The uncertainty principle. Ballentine [3, p. 364], like other ensemble theorists [99] regards this principle as a statistical dispersion principle. The quantities $\Delta x$ and $\Delta p_{x}$ are obtained by measuring $x$ and $p_{x}$ on many similarly prepared systems and determining the standard deviations. It must be said immediately that this is a totally acceptable statement of the principle. It may, though, be thought to be quite close in spirit to a quite different idea (seen for example in Popper $[65, \mathrm{p}$. 20]) that the 
distributions of $x$ and $p_{x}$ may be of values held before measurement. This view could, of course, only be held by a PIV theorist; it would be anathema to anyone holding an orthodox position, or, indeed. anyone supporting an ensemble interpretation of any type other than PIV.

Indeed, in this section of his paper, Ballentine is quite clearly supporting PIV. He considers [3, p. 365 ] the view, "the position and momentum of a particle do not even exist with simultaneously and perfectly well defined (though perhaps unknown) values". He considers that this argument is "easily seen to be unjustified" because "it rests on the almost literal identification of the particle with the wave packet (or what amounts to the same thing, the assumption that the wave function provides an exhaustive description of the properties of the particle)". (His demonstration of the "untenable nature" of such an identification using a particle incident on a semitransparent mirror would certainly be disputed by supporters of the orthodox position.)

Ballentine strongly criticises the use of Heisenberg's thought-experiment to justify the uncertainty principle; we would mention that a recent paper [147] has reformulated this thought-experiment in terms of the statistical dispersion interpretation of the principle.

Incidentally, Ballentine comments that it is "very common" for orthodox discussion of the relations to use the idea of simultaneous measurement. While this terminology is certainly not unknown (e.g., ref. [148]), it must be illegitimate for those supporting an orthodox position, since, for them, unlike PIV supporters (section 3.2.2), simultaneous measurement is not allowed. In fact, many orthodox discussions either restrict themselves to talking mathematically about distributions of $x$ and $p_{x}$ over $\psi$, or refer to $\Delta p_{x}$ and $\Delta x$ as the uncertainties of the momentum in the $x$-direction and the position in the same direction [105, p. 29]. This latter definition might be made more rigorous by explicit mention of a probability distribution over (potential) experimental results, which itself may be tested as in Ballentine's statement of the principle.

(3) Joint probability distributions, Almost implicit in the idea of a PIV-ensemble is that there should exist a joint probability distribution $P\left(x, p_{x}, \psi\right)$ which would yield the correct expectation values, and also give the usual single-variable distributions as marginals [3, p. 372]. It is very well-known that the so-called Wigner distribution [149] fulfils such a role mathematically, but cannot be a genuine probability distribution as it takes negative values.

Ballentine gives a good account of the 1970 situation, including the result of Margenau and Cohen $[65$, p. 71$]$ that there cannot exist "a joint probability distribution such that the averages of all observables can be calculated by a phase-space average as in classical statistical mechanics" [3, p. 373]. Ballentine comments, "with the wisdom of hindsight, we should not be surprised... for if it were otherwise, then quantum mechanics could be expressed as a special case of classical mechanics. Once this is stated, the other work cited by Ballentine (and the considerable amount of work performed since) would seem to be, perhaps, of much mathematical importance, but not of comparable conceptual interest. In his conclusions, Ballentine [3, p. 367] merely notes: "It is possible to extend the formalism by the introduction of joint probability distributions for position and momentum."

(4) Measurement (and the projection postulate). Ballentine [3, pp. 368-372] considers these topics in depth. In his study of the orthodox approach, he brings out the problems of the projection postulate fully. He concludes: "None of the attempts to solve this problem using some form of reduction of state vector are satisfactory." (Later his distaste for the postulate was to become even more intense $[39,150]$.

Ballentine's explanation [3, p. 368] of how an ensemble interpretation tackles the measurement problem is, "the probability of the apparatus 'pointer' being $\alpha_{r}$ at the end of an experiment is $|\langle r \mid \psi\rangle|^{2}$. That is to say, if the experiment were repeated many times, always preceded by the same state 
preparation for both object and apparatus, the relative frequency of the value $\alpha_{r}$ would be $|\langle r \mid \psi\rangle|^{2}$. That this should be identified with the probability of the object having the value $r$ for the dynamical variable $R$ immediately before interacting with the apparatus II, is just the criterion that II functions as a measuring apparatus for $R$." It seems fairly clear that these comments must refer to a PIV interpretation: otherwise one cannot talk of an object "having" a value $r$ before measurement, or even the probability of such. (We thus assume that the word "probability" represents a relative frequency.) Unless the statement is read this way, there seems little if any difference between it, and the corresponding orthodox statement, and such a difference is definitely implied.

He adds [3, p. 371] that "no such problem [as the reduction of state vector] occurs in the Statistical Interpretation in which the state vector represents an ensemble of similarly prepared systems", and, indeed [3, p 379] that "under the more modest assumption that a state vector represents [such] an ensemble, the measurement process poses no particular problems". These comments seem to be less sensitive to the type of ensemble interpretation, and these views will be analysed in section 5 .

(5) Wave-particle duality. Ballentine [3, p. 362] asks whether an ensemble interpretation may explain such phenomena as interference and diffraction. His answer (in the affirmative) follows Duane [42], as revived by Landé [41]. In discussion of electron diffraction "there is no need to assume that an electron spreads out, wavelike, over a large region of space ... Rather it is the crystal that is spread out, and the electron interacts with the crystal as a whole through the laws of quantum mechanics". (Note that, in even asking the initial question, the PIV assumption is being made that the electron "has" a position before interaction.)

(6) "Paradoxes". Study of the various "paradoxes" of quantum theory is important for ensemble supporters. Ballentine [135] says, "a person who held the . . . individual interpretation would not reject the predictions of the ... ensemble interpretation, and it is only by analysing suitable examples (e.g., the EPR argument, Schrödinger's cat, or Einstein's discussion of the classical limit) that the weakness of the [individual] interpretation becomes apparent". In ref. [3], Ballentine discusses EPR (p. 362), Schrödinger's cat (p. 369), and Bell (p. 377), and these arguments will be analysed in section 6.

\subsection{Developments of the ensemble interpretation}

In this section we consider those discussions of ensembles which may broadly be regarded as nonstochastic additions to the formalism; broadly stochastic additions are considered in section 4.6, and those articles essentially providing analysis or criticism in section 5.

Young [151] has put forward a detailed analysis of "quantum mechanics based on position". Such concepts as "state", "dynamical variable", and "measurement" are regarded as "problematic concepts" based on "empty claims and definitions masquerading as postulates" which "QM can do without". Such experiments as Stern-Gerlach and Franck-Hertz are discussed without their aid, and a semiclassical account is given of the interaction of radiation with matter. "The result" says Young "is a particularly compact formulation of QM" which "assumes less, and derives more than is customary". In particular "it is immaterial whether quantum 'dynamical variables' exist which are 'measured' by these experiments". Young explicitly identifies $\psi$ with an ensemble. Indeed he appears to agree fairly thoroughly with a PIV approach: "[M]omentum, angular momentum (component) and energy are constants of the motion before the 'measurement'. Position, though not a constant of motion . . . is slowly varying", but it might be interesting to investigate how essential these assumptions are. (One might also consider whether there is a relation to the remarks of Bell reported in section 3.2.2.)

Buonomana [152] has developed an account of quantum mechanics as a "nonergodic classical 
statistical theory" differing from that of Ballentine only in its ergodic properties. It is an explicitly hidden-variables theory in which the state of a measuring apparatus does not relax between measurements but is a stable function both of its prior state and of the state of the particle just measured. The outcome is that, in an experiment testing Bell's inequalities, for example, the results of a single run might support the inequalities and disagree with quantum theory, but an average of many runs would always agree with quantum theory. (It is suggested that this might explain the presence of both results in the literature. Buonomana [153] also suggests that such an outcome would "be consistent with the Statistical Interpretation but not with the Copenhagen Interpretation".) In this theory it is said that the usual arguments against joint probability distributions do not apply. The penalty for these gains is that very radical properties must be assigned to empty space, in which there must exist "a field or medium with stable states or memory".

Shale [154] has presented an interesting attempt to put geometric ideas at the foundation of quantum theory. One of his ideas is that between any two events on the world line of a particle, there can only be a finite number of events. Thus the conception of the continuum is, if not abandoned, at least modified. Associated with this idea is another hypothesis that coordinates of events have only probable meanings. In fact "space must be replaced by space-time and bodies by events". As to events "precedence must be given to the fundamental sort wherein particles are created or destroyed". Thus "the Universe itself may be viewed as an enormous Feynman diagram" which Shale calls "the universal diagram". This is incompatible with the Copenhagen interpretation in which we cannot know which of a pair of double slits a photon goes through. For this reason, Shale is impelled to accept "the 'statistical' interpretation of quantum mechanics".

Prosser [155] studied the behaviour of light in the vicinity of a semi-infinite plane, and for a single and double slit directly from Maxwell's equations rather than via a Huyghens construction. Calculations of energy flow result in undulations in a nearly linear trajectory, which may be interpreted as the expected diffraction or interference pattern. He went on [156] to analyse a single photon as a stable superposition of infinite waves, and thus, admittedly, not perfectly monochromatic. He was able to demonstrate not only wavelike phenomena, but also corpuscular ones - the Compton and photoelectric effects. In this theory, probability is not a primary element, but arises because of ignorance of the detailed emission and absorption processes of the emitting atom. Thus quantum theory is not complete. and $\psi$ has meaning only in relation to an ensemble of photons. A particle follows a definite trajectory. and the uncertainty relations correspond to the relationship between the range of frequencies in a wave packet and its spatial extension. Prosser suggests an experimental test of this theory, based on his prediction that, in a double-slit experiment, photons which reach the right-hand part of the screen must have passed through the right-hand slit.

Thankappan and Nambi [157] also indicate a definite trajectory for a particle. In fact, identical particles may journey from one set of coordinates (position and velocity) to another along different trajectories, and an ensemble may thus be built up. The source of these ideas is a modification of the Feynman formulation [158]. Feynman makes use of a mathematical summation over virtual paths, which leads to the usual Schrödinger equation. In ref. [157], the analogous summation is a physical one along real paths.

Das [159] has analysed a generalised Klein-Gordon equation in the study of meson field, following Yukawa [160], who had obtained two equations for the scalar field, one for space-time and a second for momentum-energy space. Das has taken these equations together, producing a 7-dimensional space, and studied the Fourier transform of the scalar field. Such quantities as total momentum, total energy and total number may be expressed in terms of Boltzmann-type distribution functions for mesons in the 
dual phase space. Das concludes that "this view strongly supports the statistical interpretation of the quantum mechanics".

While the exact solution of the Schrödinger equation for Coulomb scattering has been known for over 60 years, the asymptotic form as $h \rightarrow 0$ was not studied fully till the work of Rowe [161] in 1987. He showed that, in that limit, "the classical reality to which the quantum mechanical treatment limits is a statistical ensemble of individual single-particle scattering orbits. The quantum mechanical probability becomes a statistical probability for an ensemble of classical orbits . . . To put it no more strongly, the simplest interpretation of the quantum mechanical formalism when $h \neq 0$ which agrees with the required interpretation in the $h \rightarrow 0$ limit is the statistical interpretation."

We now turn specifically to the areas of the uncertainty principle, and joint probability distributions (or, to put it another way, comparison of classical and quantum probability spaces). These topics are also very much linked with study of the Bell inequalities in section 6.4. The latter connection has been particularly stressed by Fine [162] who has stated, and demonstrated, that "hidden variables and the Bell inequalities are all about ... imposing requirements to make well defined precisely those probability distributions for noncommuting observables whose rejection is the very essence of quantum mechanics."

Hall [163] rewords Fine's statement to say that "the positivist and the realist disagree in their definitions of joint probability", where "positivists" are "proponents of the completeness of the Copenhagen interpretation", and "realists" support "interpretations in which the 'quantum' probabilities are generated by an underlying 'classical' probability structure". Hall then claims that the "positivist" is forced to modify the concept of joint probability by accepting a weakened form of the "contradictory inference" [164] (or theorem of complete probability), explicitly, $W(A \mid B)+W\left(A^{\prime} \mid B\right) \leq$ 1 , where $W(A \mid B)$ is "the probability that $A$ will be verified by a corresponding yes/no experiment, given that $B$ has been found to be true upon performance of a suitable yes/no experiment with the system in a state $W^{\prime \prime}$ and $A^{\prime}$ is falsified [verified] by a yes [no] result in a yes/no experiment on $A$. Classical probability would give us an equality sign, of course, but, according to Hall, quantum theory only gives it in the case where $A$ and $B$ commute. This tells us that "in general no matter what physical proposition $B$ is tested and found to be true for some system, there will be some 'complementary' proposition $A$ which cannot be said, even in principle to be either true or false" so this inequality "provides a mechanism for incorporating complementarity into ... probability".

Hall thus explicitly contradicts Ballentine [165], who argues cogently that "the axioms of probability theory are satisfied by the formalism of quantum mechanics".

Cohen, whose early work was reported in the previous section, has continued to study probability in classical and quantum physics. Working from a classical probability distribution, he has been able to produce [166] an infinite number of "state functions" given by

$$
g(x)=(2 \pi)^{-1 / 2} \int_{-\infty}^{\infty}[P(\alpha)]^{1 / 2} \exp [\mathrm{i} S(\alpha)+\mathrm{i} x \alpha] \mathrm{d} \alpha,
$$

where $P(\alpha)$ is the quantum mechanical momentum distribution, $\alpha$, the momentum, and $S(\alpha)$ is an arbitrary real function. He hence obtains, still from classical theory, the operator method, the formula for the expectation value, and, using the discrete case, the apparatus for eigenvalues, quantisation, and the Born rule. The method may be extended to observables other than momentum. It is the richness given by the arbitrary $S(\alpha)$ which appears to generate the wide variety of probability functions needed for quantum theory, that cannot be obtained from the standard theory. 
He also shows that, contrary to expectation, joint probability distributions with the correct marginals may be obtained in quantum theory, though they will not yield the correct quantum mechanical expectation values. Unlike distributions of the Wigner type discussed in section 4.4 , these distributions will not in general be bilinear in the state function.

Ioannidou [167] has claimed to give a statistically founded derivation of the Planck formula by considering a classical statistical ensemble of one-dimensional simple harmonic oscillators of common frequency $\nu$ at temperature $T$. Ioannidou suggests that, since matter is not continuous, there are occasions when the approximation to a continuum in phase-space becomes unsupportable, and hence he defines a measure of the elementary range in phase space, $\sigma$. He deduces that $\varepsilon=\sigma \nu$, where $\varepsilon$ is an "elementary" energy, and, by comparison with Planck's $\varepsilon=h \nu$, suggests that $\sigma \simeq h$. He concludes that $h$ and $\varepsilon$ characterise a statistical ensemble of particles, and not an individual particle, and that the uncertainty relation is a statistical dispersion principle, but it is not perhaps quite clear how genuinely independent of the usual arguments his own equations are.

Sutherland [168] has approached the question of a joint probability distribution for position and momentum from the point of view of conditional mean values over an ensemble of particles with different momenta and energies. From comparison of the classical formulation with both the Schrödinger and Klein-Gordon equations, he is able to postulate that

$$
\left\langle A^{n}\right\rangle_{r, t}=\operatorname{Re}\left\{[1 / \psi(r, t)] \hat{A}^{n} \psi(r, t)\right\} .
$$

where \langle\rangle$_{r . t}$ denotes the conditional mean value for a given position $r$ at a time $t, A$ is any observable, and $\hat{A}$ is its corresponding operator. From this equation he deduces a form for $P(x, p ; t)$, the joint probability distribution

$$
P(x, p ; t)=\operatorname{Re}\left(\psi(x, t) \int_{-x}^{x} \exp (-\mathrm{i} \Gamma p)(x-h \Gamma, t) \mathrm{d} \Gamma\right)
$$

a form previously introduced by Margenau and Hill [169]. In a later paper [170) Sutherland considered the uncorrelated distribution

$$
P(x, p ; t)=|\psi(x ; t) \phi(p ; t)|^{2}
$$

the only positive-definite form known, but apparently quite inappropriate in the classical limit. He questions this latter conclusion, suggesting that "the . . classical correlations come from the form of the wave function itself rather than from any joint distribution".

Other important work in this area can be mentioned only very briefly. Srinivas [171], after commenting that "the differences between the various interpretations of quantum theory arise out of the differences in the interpretations of the probabilities predicted by the theory", concludes that "the various joint and conditional probabilities in quantum theory. . . are not related to each other as in classical probability theory". Collins [172], rather in contrast, concludes that "the formalism of quantum theory is . . . just a Hilbert space formalism for classical probability theory and that not only is it possible to represent a probability density as the absolute square of a wave function but such a form is necessary in order to correct a defect in classical probability theory" (the defect being to allow negative values).

Among the foremost writers on quantum probability have been Gudder [173] and Pitowsky [174]. 
Here we merely report a recent investigation of Gudder [175], in which, aiming for a unification of general relativity and quantum mechanics, he presents a "generalization or extension" of the latter, in which the usual Feynman postulates are used, together with the addition that "the probability of an event for a measurement is the 'sum' of the probabilities of the outcomes composing it", thus leading to a "realistic quantum probability theory".

On Pitowsky we report the conclusion of a recent paper [176]. He suggests that "the problem of realism ... is irrelevant to the understanding of quantum mechanics. One can ... conceive of quantum probabilities as resulting from fixed properties. One can ... decline to do so... In classical as in quantum physics, both alternatives are compatible with the experimental results." Pitowsky criticises Bohr's interpretation for "unnecessary focus on the metaphysical problems" and suggests "only two coherent alternatives exist." The first is that " interference' and 'collapse of wave function' are physical processes caused by unknown mechanisms". In this case "one can hope to control and manipulate these processes and obtain results which transcend or even contradict quantum mechanics". The second is that "'interference' and 'collapse...' are names for the fact that the probability theory associated with quantum theory is nonclassical". Which is correct, Pitowsky believes, is "in large part empirical ... if [the first] is the correct one we shall find out sooner or later".

We mention the argument of Gibbins [177] that, from the point of view of quantum logic, the understanding of the uncertainty principle must be "very much like that of the statistical interpretation", and "a term such as statistical dispersion principle would be more appropriate". Jammer [178] has made a few comments, mainly historical, on this paper, and Busch and Lahti [179] have criticised it on the basis of their own idea of "unsharp" measurements to be discussed in section 5.

Lastly in this section we mention the paper by Park and Band [180] which, without containing many new ideas, provides another trenchant attack on orthodox views, which, it claims "have been absorbed uncritically into the fabric of information-theoretic statistical mechanics, with the result that quantum statistics is not yet truly as well grounded as a cursory survey might suggest.

\subsection{Developments in stochastic interpretations}

In this section we sketch a few of the interesting developments in stochastic interpretations since Jammer's account [4, ch. 9] was published in 1974. (There is, of course, no rigid dividing line between this section and section 4.5.) Of other accounts, Gudder's book [123] of 1979 is titled Stochastic Methods in Quantum Mechanics but the title is interpreted broadly to include, for example, quantum logic, the operation approach of Ludwig [181], Davies [182] and Lewis [183], and quantum field theory, as well as some of the type of material discussed here.

Jammer opens his account $[4$, p. 418] by saying: "The main objective of the stochastic interpretations of quantum mechanics has been to show that quantum theory is fundamentally a classical theory of probabilistic or stochastic processes, and as such conceptually of the same structure as, say, the Einstein-von Smoluchowski theory of Brownian motion." A reply from de la Peña and Cetto [184] is: "This may sound as astonishing as the inverse assumption that Brownian motion is fundamentally a quantum theory ..."

What is true [185] is that nearly all of Jammer's account does deal with connections, at different levels of sophistication, between the Schrödinger and the diffusion equation, as studied by such workers as Fenyés [186], Nelson [187], and de la Peña and Cetto [121] themselves. As representative of this work, we summarise ref. [121]. The aim of the paper is to produce and examine a set of partial differential equations for the statistical behaviour of a more general stochastic system than that required 
for Brownian motion. Then one choice of parameters may give the Brownian motion solution, but another may yield the Schrödinger equation.

The particles considered behave stochastically because of a stochastic surrounding medium acting in addition to the applied forces. From kinematic considerations, the two basic equations for a volume element may be obtained as

$$
m\left(D_{\mathrm{c}} \boldsymbol{v}-\lambda D_{\mathrm{s}} \boldsymbol{u}\right)=\boldsymbol{F}^{\prime}, \quad m\left(D_{\mathrm{c}} \boldsymbol{u}-D_{\mathrm{s}} \boldsymbol{v}\right)=\boldsymbol{F}
$$

Here $\boldsymbol{v}$ is the average velocity of the volume element and $\boldsymbol{u}$ is a "stochastic velocity" representing random motion of particles in the volume element. In rather similar fashion, $D_{c}$ and $D_{s}$ represent "systematic [i.e. usual] and stochastic derivative operators". $F^{-}$and $F^{-}$represent those parts of $\boldsymbol{F}$, the mean value of the force on the particles of the system, that are invariant, and change sign respectively under time reversal. $\lambda$ is a free parameter. Then the first equation represents a stochastic generalisation of Newton's second law, which goes over into the law itself in the nonstochastic limit when $\boldsymbol{u} \rightarrow 0$. The second equation represents a continuity condition; in the nonstochastic limit, both sides tend to zero.

If we introduce $\psi_{q}$, equal to $\exp \left(w_{q}\right)$ where $w_{q}$ is defined using

$$
\boldsymbol{v}_{q}=\boldsymbol{v}+(-\lambda)^{1 / 2} \boldsymbol{u}=2 \varepsilon D \boldsymbol{\nabla} w_{4}-(e / m c) \boldsymbol{A}
$$

then, working to second order, the Schrödinger equation is obtained, provided one takes for the two free parameters, $D=\hbar / 2 m$, and $\lambda=1$. The continuity equation then indicates that $\psi^{*} \psi=\rho$, the density of particles, and the first equation is just a Fokker-Planck equation.

On the other hand, with $\lambda=-1$, the classical Langevin equation for Brownian motion is recovered, with $D$ the diffusion coefficient. Such differences between the two equations as the absence of interference in the Brownian case may be explained. So de la Peña and Cetto conclude [184] - and it may be regarded as their response to the remarks of Jammer quoted above - "quantum mechanics may be interpreted as a Markov process, but irreducible to a Brownian type stochastic motion". So "the electron follows a stochastic trajectory, broadly reminiscent of Brownian motion, but at the same time differing essentially from it, due to the entirely different nature of the background in which electrons and Brownian particles move... Schrödinger's equation emerges as a result of a process of linearization of a system of coupled nonlinear differential equations that describe the average flow of mass and impulse through any volume in space" [188].

The same authors report [184] that the above type of theory has been used to study the two-particle problem [189], spin [190], relativistic generalisation [191], radiative corrections [192] and barrier penetration [193]. One interesting point [188] is that eigenstates of sharply defined energy can never be physically meaningful - a point that does not, of course, necessarily tell against the theory.

From about 1977, de la Peña and Cetto [188] considered the above phenomenological theory established, and turned their attention to the physical cause of stochasticity, and hence to the theory of stochastic electrodynamics (SED), which they call [184], "not the only conceivable possibility for a fundamental stochastic theory of matter, but undoubtedly the best explored one". In SED, the stochasticity is explained by assigning a real character to the random-point radiation field or vacuum field, which is usually treated as a virtual or formal field. The basic ideas emerged in the mid-1950s [194-197], but the main development was in the work of Marshall in the 1960s [198] (reported briefly by Jammer [4, p. 431]), which was followed up by many workers $[199-203,184,185]$.

We briefly describe the basic development, mainly following ref. [188]. In a nonrelativistic approach, 
the equation for an electron will be

$$
m \boldsymbol{x}=\boldsymbol{F}+m \tau \dddot{\boldsymbol{x}}+e \boldsymbol{E}(t),
$$

where $\boldsymbol{F}$ is the external force, the second term is an approximation to the radiation reaction force, and the third represents the background radiation field as a stochastic electric field. The stochastic field has the properties that $\langle E\rangle=0$, and the average energy per normal mode is $\hbar \omega / 2$, or, in other words, the spectral energy density is given by

$$
\rho(\omega)=\left(\hbar \omega^{3}\right) /\left(2 \pi^{2} c^{2}\right)
$$

Thus $\hbar$ enters the theory as a measure of the field fluctuations; it is always related to fluctuations, and this is how the uncertainty relations must be interpreted [184]. To avoid the presence of the third derivative causing runaway solutions, an asymptotic condition $\ddot{x} \rightarrow 0$ as $t \rightarrow 0$ may be introduced, and eq. (4.7) then becomes (to first order in $\tau$ )

$$
m \ddot{x}=F+\tau(\dot{x} \cdot \nabla) F+e E_{\mathrm{m}}(t),
$$

where $\boldsymbol{E}_{\mathrm{m}}$ is a modified vacuum electric field with spectral energy density

$$
\rho_{\mathrm{m}}(\omega)=\rho(\omega) /\left(1+\tau^{2} \omega^{2}\right)
$$

Equation (4.9) is called the Braffort-Marshall equation.

There are two important differences from the analogous equation for Brownian motion, the Langevin equation. First, here the radiation reaction force is proportional to $\ddot{x}$, or in this approximation to $(\dot{\boldsymbol{x}} \cdot \boldsymbol{\nabla}) \boldsymbol{F}$; the drag term for Brownian motion is proportional to the velocity. Secondly, the Brownian analogy to $\rho_{\mathrm{m}}(\omega)$ is a constant corresponding to white noise.

The equation may be studied in phase space, using the Liouville equation for the phase-space density $R(\boldsymbol{x}, \boldsymbol{p}, t)$,

$$
\partial \boldsymbol{R} / \partial t+\hat{L}_{\mathrm{T}} R=0
$$

where $\hat{L}_{\mathrm{T}}$, the Liouvillian operator, is given by $\dot{\boldsymbol{x}} \cdot \boldsymbol{\nabla}+\dot{\boldsymbol{p}} \cdot \boldsymbol{\nabla}_{p}$. Averaging over the ensemble, and hence over $E_{\mathrm{m}}$, we obtain (to first order in $e^{2}$ and $\tau$ ) an integro-differential equation for $Q$, the average of $R$,

$$
\partial Q / \partial t+\hat{L} Q=-e \nabla_{p} \cdot E_{r} Q
$$

where $\hat{L}$ is the nonrandom part of $\hat{L}_{\mathrm{T}}$ and $E_{\mathrm{r}}$, an averaged effective field given by

$$
\boldsymbol{E}_{\mathrm{r}}=e \int_{0}^{t} \mathrm{~d} t^{\prime} \exp \left[-\hat{L}\left(t-t^{\prime}\right)\right]\left\langle\boldsymbol{E}_{\mathrm{m}}(t) \boldsymbol{E}_{\mathrm{m}}\left(t^{\prime}\right)\right\rangle \cdot \boldsymbol{\nabla}_{p} .
$$

For Brownian motion, the corresponding Fokker-Planck equation has a diffusion term on the right-hand side of the form $D \nabla_{p}^{2}$, yielding a Markoffian process. Here there is a time-integrated term, implying a memory effect, and hence a non-Markoffian process. 
Let us now assume that the system eventually reaches equilibrium, and the distribution then becomes even in $p$. Then the equation for $Q$ becomes the Schrödinger equation in phase space, but with two additional terms. The first is due to the nonlinear nature of the external force, and so disappears for the simple harmonic oscillator; the second is a radiation term. There is another important difference with Brownian motion. In the latter, the effects of the stochastic force can only be neglected if motion ceases. For SED, it plays an important role as the system moves to equilibrium, but once equilibrium is attained, the only nonclassical terms are radiative "corrections". In this way, SED may, in some cases, reach beyond the Schrödinger equation towards nonrelativistic quantum electrodynamics, though, of course, for exact results, one requires to be correct to all orders of $e^{2}$ and $\tau$.

Indeed the simple harmonic oscillator has been studied particularly successfully [198, 203]. To order zero in $\tau$, the usual energy levels and eigenfunctions are obtained, together with a temperaturedependent phase-space probability density in the form of a sum of Wigner distributions for the various states, together with appropriate Boltzmann factors. If one works to first order in $\tau$, one obtains also the decay constant and level shifts in agreement with quantum electrodynamics.

Another interesting calculation [204] concerns the Planck distribution. If a particle in the form of a dipole oscillator moves inside a cavity filled with thermal radiation, the dipole gains a fluctuating momentum in a short time interval, but the particle loses momentum due to the dissipative force of the radiation pressure. In equilibrium $\left\langle p^{2}\right\rangle$ must be constant, and electromagnetic theory gives an expression for the spectral density of the electromagnetic field - the Rayleigh-Jeans law. If we now add in the fluctuations of the vacuum field, though, and provided the zero-point energy is taken to be $\hbar \omega / 2$ per normal mode, we obtain the Planck distribution.

Other successes of SED include work on van der Waals forces, the free radiation field, the free particle, the infinite square well, and the electron spin; they make it "difficult . . to believe that all the qualitative and quantitative results obtained are mere accidents" [184]. Two major problems are in atoms, where there is nonrecurrence, and hence nonergodicity [205], and the anharmonic oscillator [185], where some results fail to agree with quantum theory.

Let us briefly review other recent stochastic theories. Santamato [206] has discussed what he calls the "generalised stochastic approach" to quantum mechanics, associating complex-probability measures, which obey the rules of classical probability theory, to real-valued diffusion processes. In this approach, a quantum particle of mass $m$ undergoes a complex-measured real-valued Brownian motion governed by a stochastic differential equation involving a generalised Wiener process with zero mean and covariance. A physical interpretation can only be given if parameter $\varepsilon$ is real, the Brownian limit, or if it is imaginary, the quantum limit. Santamato shows that the requirement of gauge invariance is all one needs to obtain a one-to-one correspondence between this approach and standard quantum theory. He says, "one thus obtains a consistent physical interpretation of the present stochastic theory, provided we admit that all measurements on a spinless quantum particle can be reduced to position measurements. and that two successive position measurements cannot be made on the same particle". ideas which, he says, are "in perfect agreement with the current statistical interpretation of quantum mechanics" (and he refers to our ref. [3]).

In another investigation [207], Santamato examines a possible connection between the translation law for vector lengths in special and general relativity, and quantum interpretation. By relaxing the assumption that vector lengths are invariant, and assuming a quantum particle undergoes a relativistic random motion in space-time, a completely classical interpretation of the Klein-Gordon equation may be obtained. The probabilistic concepts used for the motion are themselves purely classical, no quantum interference occurring. $\psi$ represents, not a probability amplitude, but the information needed to 
describe a Gibbs ensemble. Thus, Santamato suggests, "the statistical interpretation is extended to the relativistic domain". He also says that the difficulty of the Klein-Gordon as a one-particle equation is removed, since "a constant statistical interpretation can be obtained in spite of the indefinite sign of the "spatial charge density""; and the same interpretation can also be applied to neutral particles.

Hestenes [208] has developed an interpretation of spin and uncertainty in quantum theory, the "logical conclusion" of which, he says, would be "a stochastic theory of motion of a point particle". It is based on the Pauli electron theory, which reduces to the Schrödinger theory in the absence of a magnetic field. The Pauli wave function can then be written as a superposition of two orthogonal spin eigenstates, so Schrödinger theory must be regarded as describing an electron in an eigenstate of spin. Hestenes attempts to develop a model of spin "as a dynamical property of electron motion", so that spin and magnetic moment "describe a circulation of electron mass and charge". In the ground-state of the hydrogen atom, there is then an average (purely orbital) angular momentum $\hbar$, as in the Bohr theory. This enables the uncertainty relations to be explained "by interpreting electron spin as a minimum orbital angular momentum". If the region of confinement is diminished, the momentum must be increased to maintain the angular momentum.

Hestenes reports that, for the above to be plausible, "it appears necessary that the wave function represents a statistical ensemble", and there should be a phase-space distribution function. An important quantity in this analysis is $\beta$, which arrives "unsolicited" and "provides the major obstacle to a complete interpretation of the Pauli theory". It is at least suggested that $\beta$ describes energy fluctuations in the ensemble "by specifying an average deviation in the energy of ensemble members from the energy associated with the average momentum". Also "eigenfunctions ... can be identified with ensembles for which the corresponding eigenvalue is a common property of each member ... [E]nergies of atomic states have finite natural widths not determined by sharp energy eigenfunctions, so the ensembles defined by energy eigenfunctions are too narrow to fully represent the physical properties of atomic states, and a quantum theory of radiation had to be developed to rectify this. The point is that eigenfunctions are not the fundamental representatives of physical states they are sometimes made out to be. Fully satisfactory criteria for determining the ensemble which best describes a give physical system are yet to be developed."

Hestenes supports the usual arguments of ensemble theorists for the uncertainty principle being scatter relations, and diffraction theory being explained by the Duane [42] argument. The electron is "regarded as a particle", its property of extension in space being a property of the ensemble. (In conformity with de Broglie, though, individual electrons may exhibit periodicity in their motion.) Since "all stationary electron states produce fluctuating electromagnetic fields", Hestenes can discuss a number of phenomena such as van der Waals forces and electromagnetic vacuum fluctuations along analogous lines to those of SED theorists, though from a somewhat different point of view. Hestenes has also applied similar notions to the Dirac theory [209].

We briefly mention a different approach by Moore [210], which he terms "stochastic mechanics" in contrast to the diffusion model which he calls "stochastic quantisation". Stochastic mechanics is a direct extension of classical mechanics to open systems. The classical particle has its motion modified by interactions with the surroundings, and the trajectory may therefore be described by a stochastic process.

Lastly, and again more briefly than it deserves, we mention the work of Prugovečki [211]. In what he calls "the stochastic quantum mechanics approach to the unification of relativity and quantum theory", he shows that "two stochastically space-like separated regions of spacetime cannot be viewed... as absolutely unrelated". Since such incompatible observables as position and momentum are allowed to 
"possess" simultaneous stochastic values, such "paradoxes" as EPR are claimed no longer to exist. The price that has been paid is that "stochastic quantum mechanics does not allow for mutually exclusive possibilities so that the standard quantum logic of yes-no questions requires modification".

\section{Ensemble interpretations; analysis}

\subsection{PIV interpretations; general remarks}

We begin our survey of comments on, and criticisms of, ensemble interpretations, by concentrating on the PIV assumption, the idea that all observables have values prior to any measurement. It has been shown, particularly in sections 4.3 and 4.4 , that this idea has been very powerful in the advocation of the ensemble interpretation, even though it is sometimes not explicitly acknowledged.

It has to be admitted that - if one is able to accept it - it solves many of the conceptual dilemmas of quantum theory. The problem of wave-function collapse is avoided. The premeasurement value is available to become the measuremental result (though, of course, PIV theorists are not obliged to require that either the initial or any repeated measurement actually gives the PIV). Thus behaviour of the wave function at a measurement becomes very much secondary. Fine $[125$, p. 48; 124, p. 979] quotes Einstein saying in a 1935 letter to Schrödinger that he "could not care less" whether the wave function in one wing of an EPR experiment, following a measurement on the other, is an eigenfunction of the appropriate observable. It is fairly clearly that the EPR and Schrödinger's cat "paradoxes" become straightforward under a PIV interpretation. (See section 6.)

We shall also mention a few other ways in which PIV assumptions lead to attractive ways of looking at quantum theory. As we said before, Popper is more straightforward in his use of these ideas than many other writers, so it is convenient to use his works to point out ideas that may be implicit in other accounts. For Popper, the "great quantum muddle" [65, p. 19] consists of treating a distribution function as a physical property of the elements of the population, with which, Popper says, it has "hardly anything to do". In other words, $\psi$ is treated as a property of a system. For Popper, a believer in PIVs, it seems obvious that such an idea is "muddled", but, without PIVs, one has only a distribution of postmeasurement values; before a measurement, $\psi$ cannot be considered as a distribution.

PIVs enable Popper [65, p. 34] to dismiss the wave-function collapse as merely the "mental process" of selecting the appropriate subensemble. Such selection is always trivially present in any discussion of repeated measurement. One selects those systems for which the first measurement gave a particular result. But this does not make any contribution towards solving the measurement problem. The questions remain: why did the initial measurement give the particular result, and how did the measurement process affect the system (affect the wave function in any orthodox interpretation)? Only with PIVs is the situation so simple that the mental process of subensemble selection is all that is required [87, p. 246].

The fruits of the PIV approach are thus enormous, and we have already suggested (section 4.4) that the simplest argument against it, from the uncertainty principle, does not succeed. (See also section 5.7.) Nevertheless, there are many arguments against so simple an approach, and we shall review them for the rest of this section and the next. (See also section 6.4 for additional arguments.)

Before this, though, we should clarify one point. A similar, though less extreme, position to PIVs is where observables do not necessarily "have" values prior to measurement, but the value that any observable will take, if it is measured, is fixed. This would, of course, usually be described as a 
hidden-variables theory, which one might wish to be local and/or deterministic. It was this type of theory, rather than the more restricted PIV type, that Bell examined in his famous paper [21]. He was able to show initially that "there is no difficulty in giving a hidden variable account of spin measurements on a single particle", but of course the same did not apply to the coupled EPR pair. Most of the arguments made in this section apply only to the PIV case, not to the more general situation; indeed, of course, successful hidden-variables theories have been constructed [19], though these are limited (in any requirement of locality) precisely by Bell's result.

It is interesting to consider PIVs specifically, because talk of definite trajectories in ref. [3], and many articles deriving from it, require that position and momentum actually "exist" in the absence of any measurement, not just that hidden variables exist which will produce the appropriate results if the measurement is actually made.

Now we turn to the problems of PIVs, on which Fine comments [125, p. 41], incidentally, that "the so-called interpretation has been faced with difficulties since 1935, difficulties known to Einstein and simply ignored by him". The initial challenge to Einstein came from Schrödinger [212], in a section of his paper titled, Can one base the theory on ideal ensembles? His first problem relates to radioactive decay and is as follows. If $|\psi|^{2}$ represents an ensemble of systems, then PIV ideas will tell us that some members of the ensemble have positions inside the barrier, though their total energy is less than the barrier energy. Schrödinger takes for another example [212, p. 156], angular momentum. One may imagine that the angular momentum about a particular point $O$ takes an allowed value $[l(l+1)]^{1 / 2} \hbar$, but about another point $\mathrm{O}^{\prime}$, "unacceptable values occur", leaving Schrödinger to conclude that "appeal to the ensemble is no help at all".

Another example is for the energy of an oscillator, where $\psi$ takes on nonzero values for distances $x$ such that again the potential energy must be greater than the energy eigenvalue. Similarly [212, p. 164], Schrödinger considers a system for which the energy is equal to the sum of squares of canonically conjugate quantities, $E=p^{2}+q^{2}$. Independent measurements of $E, p$ and $q$ yield quantities which cannot be combined to obey the equation, yet that is what the PIV idea requires.

Blokhintsev [17] has put forward analogous arguments for the helium atom, arguments by which, in Jammer's words [4, p. 164], "the assumption that a particle has simultaneously well-defined values of position and momentum, even though these may be unknown and unobservable, can be rejected independently of the complementarity interpretation". Jammer translates the argument to the case of the hydrogen ground state, showing that, on a PIV model, almost $25 \%$ of the electrons would have potential energy greater than total energy.

Many other trivial examples are well-known [1]. For example, for a spin-3/2 particle (and putting $\hbar=1$ ), values for $S_{x}, S_{y}$ and $S_{z}$ cannot be selected from $\pm 3 / 2, \pm 1 / 2$ to make the sum of squares equal to $S(S+1)$, that is, $15 / 4$. And, for a spin-1/2 particle, if $S_{z}$ and $S_{x}$ have values of $\pm 1 / 2$, on a PIV model, $(1 / \sqrt{2})\left(S_{x}+S_{z}\right)$ must equal $0, \pm 1 / \sqrt{2}$, not $\pm 1 / 2$ as it should. These problems may indeed be trivial, but they do not appear to be faced up to by PIV theorists.

We now turn to a more sustained attack on PIV theories, that of Feyerabend [87], on Popper in particular. We recall Feyerabend's arguments from section 3.3.3 that, from the quantum postulate, that is, arguing from experiment, one is able to show that "intermediate states" ("classical" states for the motion of individual systems) cannot be well-defined [87, p. 198].

Feyerabend $[87$, p. 200] characterises ensemble theorists as claiming that, when a second slit is opened, "the change of path of the individual electron is not in need of explanation. What can be explained . . . is the emergence of a new stochastic process that leads to a new interference pattern. This position is indeterministic, as it admits the existence of uncaused individual changes and its indetermin- 
ism is about as radical as that of the Copenhagen point of view. It also shares with that point of view its emphasis on the experimental situation: predications are valid only for certain experimental conditions... However it differs from the Copenhagen point of view insofar as it works with well-defined states and trajectories."

Here Feyerabend makes the crucial point - and it is one, he says, Popper has admitted in private communication - that, in this theory, "the conservation laws are valid only for large ensembles of particles". Historically, of course, this is a particularly interesting point. Prior to the development of (modern) quantum theory, Bohr, Kramers and Slater [213] put forward their own theory in which conservation of energy was statistical, but these authors withdrew their theory after the experiments of Bothe and Geiger [214], and of Compton and Simon [215] were accepted as giving clear evidence that energy and momentum are conserved in each individual process. It might be added that elementaryparticle physics also relies on just this assumption, and its own consistency provides more evidence in the same direction. The challenge, then, according to Feyerabend, is for ensemble theorists to provide alternative explanations of all these experiments. (For other relevant portions of this article, see p. 217 and, on measurement, pp. 245, 246 of ref. [87].)

A rather distinct set of arguments concerns the question of subensembles (Gillespie, unpublished, 1976). Consider again an ensemble, $E$, of spins $1 / 2$ in an eigenstate of $S_{z}$. According to PIV theory. half have $S_{x}=1 / 2$ and half $S_{x}=-1 / 2$, so this gives two subensembles $E_{+}$and $E$. Now consider ensembles in eigenstates of $S_{x}, D_{+}$and $D$. One might consider attempting to identify $E_{+}$and $E$ with $D_{+}$and $D_{-}$, but this cannot be right, since $\left\langle S_{z}\right\rangle$ is zero for $D_{4}$ and $D_{-}$, but not for $E_{+}$and $E$. Yet the relationship remains obscure.

One could approach the question from another point of view, one which is of historical interest, as well as conceptual, because it appears to have been the real reason for von Neumann's rejection of hidden variables, and not his famous "proof" [14, pp. 303-305;216; 4, p. 266]. By performing a measurement of $S_{x}$ on ensemble $E$ above, and separating the systems according to result obtained, one might hope to produce subensembles $E_{+}$and $E$ with definite values of $S_{z}$ and $S_{x}$. Yet experience tells us that this is not possible; the operation will produce ensembles $D_{*}$ and $D_{-}$instead, with values of $S_{x}$, but having "lost" specific values of $S_{z}$. This may seem to be a refutation of the PIV idea, but, of course, one can discuss the "loss" of $S_{z}$ value by interaction with hidden variables in the measuring device. This was Schrödinger's view, according to Wigner [216]. Von Neumann and Wigner [216, 217] had counter-arguments, but Clauser [218] showed that this kind of proof can never be made watertight. From our point of view, though, it is interesting that much has been thrown back onto the measurement process, rather in discordance with the PIV idea, at least in its simplest form, that the measurement may simply record values already in existence.

\subsection{PIV interpretations and the hidden-variables "impossibility" proofs}

Let us now turn to the various "proofs" of the impossibility of hidden variables [4, pp. 265-275, pp. $296-329 ; 122]$. Bell $[20,101]$ showed that these proofs were unsatisfactory since they made assumptions that were unnecessary, and certainly did not hold in the successful hidden-variables theories of Bohm [19], and Bell himself. PIV theories, on the other hand, do make just these assumptions, and therefore are ruled out by these proofs.

Bell showed, for example, that von Neumann's famous proof $[14, \mathrm{pp} .305-323]$ was nullified by his requirement that "[a]ny real linear combination of any two Hermitian operators represents an observable, and the same linear combination of expectation values is the expectation value of the 
combination", which is not necessary for hidden-variables theories, though it is true for orthodox quantum theory. Bell [20] comments: "There is no reason to demand it individually of the hypothetical dispersion free states, whose function is to reproduce the measurable peculiarities of quantum mechanics when averaged over." Here, though, the important point is that for PIVs, the requirement is fulfilled, and such states, therefore, are ruled out.

Much the same goes for the Jauch-Piron argument [219]. In the shortened form of the argument given by Bell [20], observables are represented by projection operators. Two such are $a$ and $b$, and $(a \wedge b)$ is defined as the projection onto the intersection of the two subspaces. Two of Jauch and Piron's axioms deal with the expectation values of projection operators. The first says that expectation values of commuting projection operators are additive, the second that if, for some state,

$$
\langle a\rangle=\langle b\rangle=1
$$

then for that state

$$
\langle a \wedge b\rangle=1
$$

also. This is true for quantum theory, and Bell gives a simple example [20, note 13]. He also says that there is no reason why it should apply to hypothetical dispersion free states, only to the quantum averages of these states, so the remainder of Jauch and Piron's argument fails. However the axiom above is correct for PIV states, just as for quantum states, so in this case the proof does go through, and the states themselves are disproved.

The work of Gleason [220], and Kochen and Specker [221] has also been of such interest. The latter paper shows, in the words of Belinfante [122, pp. 20,35] that "hidden variables do not tell us what are the values of certain observables. They merely tell us what values would be found if we would measure these observables in some particular way." The crux of the argument $[4$, p. 323] considers the operator $j^{2}=j_{x}^{2}+j_{y}^{2}+j_{z}^{2}$ for the state $n=2, l=0, s=1, j=1$ for orthohelium. The observables $j_{x}^{2}, j_{y}^{2}$ and $j_{z}^{2}$ are simultaneously measurable; two must give the value 1 , and the other zero. A PIV argument should provide a function that assigns to each direction the result that will be obtained when the measurement is made in that direction, but the authors show that no such function exists.

Bell stresses [101] that the argument demonstrates the contextual nature of quantum theory. If one measures $\mathrm{O}_{1}$ together with other commuting observables, say $\mathrm{O}_{2}, \mathrm{O}_{3}$ or $\mathrm{O}_{2}^{\prime}, \mathrm{O}_{3}^{\prime}$, the result obtained for $O_{1}$ will depend on whether the undashed or dashed set is measured at the same time. Srinivas [171] makes a similar point that "[unlike in classical probability theory] the conditional probabilities of a given set of events can be determined only if the sequence of all the experiments performed on the system is specified." We may also mention the work of Bach [222], based on that of Gleason, and of Kochen and Specker; Bach concludes, "there is no local ensemble representation ... [Any] ensemble representation of quantum theory necessarily is probabilistic (nondeterministic) and nonlocal; there is no meaningful extension of the concept of an ensemble formulation of quantum theory."

Bell points out [101] how well this all fits in with Bohr's insistence on the relevance of the whole experimental setup (section 3.3). Noncontextual hidden-variables theories, such as PIV, are ruled out by Kochen and Specker's argument. (See also ref. [1].)

Lastly we turn to Bell's own argument [21]. Bell's theorem has the profound implication that, irrespective of the interpretational framework, the nonlocality implied by quantum mechanically predicted correlations in EPR-type situations cannot be reduced to any local realistic description. This 
means that PIV interpretation does not help to remove the conceptual difficulty of reconciling quantum mechanics with local realism. See also ref. [1] and section 6.4. Incompatibility between quantum mechanics and noncontextual hidden-variables theories has also been demonstrated by Home and Sengupta [223] by applying Bell's inequality to the measurements of commuting observables.

Overall the difficulties for a PIV interpretation must seem practically insuperable. One study of whether at least some of the benefits of the PIV idea might be retained has been made by d'Espagnat [127]. He discusses "the assumption that some observables of some physical systems (including measuring instruments) have in all circumstances definite values [may] be made compatible with the linear laws of quantum mechanics by means of the hypothesis that not all Hermitian operators of a macrosystem correspond to observable quantities". His conclusion is negative, which he explains by saying that any "fundamental reality" must be nonseparable.

It is interesting to note the direction in which a PIV interpretation must perhaps be pushed in response to the challenges of these sections. Much of the challenge has to do with energy balance. A theory which has trajectories like the PIV theory, but which solves the energy difficulty, is just that of Bohm [19] in which the actual potential is modified by the addition of the quantum potential. The form of the quantum potential is determined as much by the experimental arrangement as by the particle itself. Thus the quantum system and apparatus form a whole in just the way Bohr had recognised (section 3.3.2). As shown by Bell [101], the theory survives all the impossibility proofs. In particular, the result of a measurement does depend on what quantities are measured simultaneously, which saves the theory from Kochen-Specker "impossibility", but goes against the spirit of the PIV idea. Nevertheless it seems about as near PIV as one can possibly get.

Interestingly, Ballentine appears to have moved in this direction. In his 1970 paper [3], Ballentine mentioned Bohm's work noncommittally [3, p. 376], and indeed commented on hidden-variables theories that "any that reproduce all the results of quantum theory exactly must, as a consequence of Bell's theorem be physically unreasonable" [3, p. 377].

In a much more recent paper [150], however, which gives no hint of a PIV theory. Bohm's theory is regarded as "a good example of a structure added within the existing framework of the statistical quantum theory". Ballentine admits: "At first sight this approach seems to go against the spirit of the statistical interpretation, since $\psi$ is no longer merely a generator of probabilities for the ensemble ... becomes an element of physical reality in the individual case." He says: "Bohm's theory is of value because it provides a model of the individual phenomena that are described only statistically by QM. The model is not likely to be unique, and generalizations should be sought." In discussing this theory (and others involving spontaneous collapse), Ballentine insists, however, "such modifications of QM are not needed to solve the 'problem of measurement'. Rather, they are needed because without modification QM cannot provide a description of individual systems, the analysis of the measurement process being only a convenient means of demonstrating this point."

We may, perhaps, then, leave this section with at least a hint that support for PIV may be on the wane, but belief that unstructured "minimal" ensembles may, in a general sense "solve the problem of measurement" may still be widespread. This is very much the starting point for the next two sections.

\subsection{The minimal ensemble}

In our discussion of Ballentine's paper [3] in section 4.4, the one feature that did not appear, necessarily at least, to lean heavily on PIV ideas was the approach to measurement. This may, then. together with the discussion of the "paradoxes" (to be considered in section 6), be regarded as 
constituting the central thrust of the ensemble interpretation. In section 5.4, then, we explicitly drop all discussion of PIVs or hidden variables in general providing structure for ensemble interpretations, and restrict ourselves to the "minimal" ensemble interpretation [2, p. 76], defined simply by the fact that a wave function represents an ensemble.

Such a position is undoubtedly tenable, but Jammer gives a warning [4, p. 441]: "[T]he thesis that quantum mechanics predicts the relative frequencies of the results of measurements performed on an ensemble of identically prepared systems is sometimes called the 'Einstein hypothesis' in contrast with what is called the 'Born hypothesis', according to which quantum mechanics predicts the probability of the result of a measurement performed on a single system. This, however, makes sense only if a distinction could be made between a theory that predicts frequencies and a theory that predicts probabilities of individual events. Such a distinction could certainly be drawn if typical quantum probabilities were not identical with relative frequencies, even if experimental procedures used for the verification of both types of prediction appeared to be identical. Most physicists deny the logical legitimacy of such a distinction."

So use of ensembles is always permissible, and may be congenial to those supporting the relativefrequency definition of probability, or others. But, remembering the "element of reality" of section 2.8 , we must examine extremely carefully all arguments suggesting that an "Einstein" interpretation is physically different from a "Born" interpretation.

We conclude this section by noting that Blokhintsev [17] has spelled out an approach to a (minimal) quantum ensemble [17, pp. 19-25] clearly distinguishing it from a Gibbs ensemble [17, pp. 15-18]. (See also ref. [4], p. 446.) In a quantum ensemble, a set of macroscopic bodies, the macrosetting, $M$, determines the state of motion of the microsystem, $\mu$. The variances $\overline{\Delta q^{2}}$ and $\overline{\Delta p^{2}}$ obey the relationship $\overline{\Delta q^{2}} \cdot \overline{\Delta p^{2}} \geq \hbar^{2} / 4$, and so, in contrast to the Gibbs ensemble, no joint probability density, $W(p, q)$, exists. To replace this, Blokhintsev uses Bohr's complementarity, though he would prefer to retitle this the "principle of exclusiveness". This tells us that "dynamic variables . . . may be divided into two mutually complementary groups: space-time ones and momentum-energy ones". Instead of $R(p, q)$, phase space, one may use either configuration space $R(q)$ or momentum space $R(p)$ yielding probability measures $W_{M}(q) \mathrm{d} q$ or $W_{M}(p) \mathrm{d} p$, the $M$ having "the same meaning as... $\theta$ (temperature) ... for a classical Gibbs ensemble; it indicates the macroscopic setting $(M)$ that governs the condition of motion for the macrosystem $(\mu)$ and hence determines its state".

Notice that Blokhintsev writes that $W_{M}(q) \mathrm{d} q$ is the "probability ... that the specific value $q$ will be found in a measurement". The italics of the last three words are ours; they emphasise that the values in the ensemble are postmeasurement, not in any sense PIV, or any sort of premeasurement distribution. This is confirmed by Blokhintsev's statement that the number of probabilities of the type $W_{M}(q)$ "exhausts the prediction of all possible measurements on a microsystem in this ensemble $(M+\mu)$ "

All probabilities can be obtained from the wave function, $\psi_{M}$. Specifically,

$$
W_{M}(a)=\left|\psi_{M}(a)\right|^{2}
$$

where $a$ may denote $p, q$ or any other observable, but $\psi_{M}(a), \psi_{M}(b)$ and so on describe the same quantum ensemble. This is a result which Blokhintsev says is "quite unexpected (from the viewpoint of classical theory)". Thus the wave function is "an objective characteristic of a quantum ensemble, but one which may be given in various representations ... $\psi_{M}$ is considered the equivalent, in quantum theory, of the classical probability $W_{\theta}(p, q) \ldots$ The wave function is not the quantity that determines 
the statistics of a quantum ensemble." Overall Blokhintsev's construction is certainly elegant, but one may ponder the real meaning of the last sentence in the light of Jammer's warning above.

We may also return to the more basic questions asked about superposition of wave functions in section 3.4. While the answer from PIV interpretations is, we have already suggested, unconvincing, it is disappointing that the minimal interpretation provides no more understanding of the superposition principle than the orthodox interpretations of section 3 .

\subsection{Measurement and collapse of wave function}

In this section we concentrate on the minimal ensemble (section 3.3). Let us review the argument on measurement from ensemble theory briefly (section 4.4) [3]. Before a measurement, the state of the system to be measured may be written as

$$
|\mathrm{I} ; \psi\rangle=\sum_{r}|\mathrm{I} ; r\rangle\langle r \mid \psi\rangle \text {, }
$$

where I denotes the measured system, $|\psi\rangle$ is its initial wave function, and the $|r\rangle$ are the eigenfunctions of observable $R$ being measured, with eigenvalues $r$. After the measurement, the state of the coupled system of measured and measuring system is

$$
|\mathrm{I}+\mathrm{II} ; \mathrm{f}\rangle=\sum_{r}\langle r \mid \psi\rangle\left|\mathrm{I} ; \phi_{r}\right\rangle\left|\mathrm{II} ; \alpha_{r}\right\rangle
$$

where II represents the measuring device, $\alpha_{r}$ is the eigenfunction of the device, and $\phi_{r}$ is the final state of the measured system corresponding to initial state $r .(\phi$, may, but, Ballentine insists, in general should not, be the same as $r$.)

The argument continues that an orthodox interpretation must now instigate a collapse from the sum of the right-hand side of $(5.4)$ to

$$
|\mathrm{I}+\mathrm{II} ; \mathbf{f}\rangle=\left|\mathrm{I} ; \phi_{r^{\prime}}\right\rangle\left|\mathrm{II} ; \alpha_{r^{\prime}}\right\rangle \text {, }
$$

a single term, corresponding to an initial state $r^{\prime}$. However, the argument continues, in an ensemble interpretation, the right-hand side of (5.5) merely tells us that the relative frequency over the ensemble of finding the measuring device in state $\alpha$, is $|\langle r \mid \psi\rangle|^{?}$.

What does this statement actually mean? Certainly, one may use this expression to calculate distributions of values of $r$ over the ensemble. Landsberg and Home [224] have analysed this approach thoroughly. They say they "have reduced the collapse postulate . . . to a normal and almost universally used postulate of statistical mechanics, which is, incidentally, also compatible with quantum mechanics", the "postulate" consisting of using a random phase in a homogeneous ensemble, so that expectation values produced from it are the same as those from a "matched" heterogeneous ensemble.

It is perhaps this type of argument that leads Squires $[115, \mathrm{p} .40]$ to say that. under the assumption that "quantum theory...tells us nothing about individual systems... [t] here are then no further difficulties in the 'interpretation of quantum theory' and the subject does not cause any philosophical problems". Though Squires considers: "This is a perfectly reasonable view, and it may be the correct one to take", it cannot be said he actually supports it: "We must not, however, go on to claim that we have solved the problems ..., We have merely ignored them. We do not only have experimental results 
for ensembles. Individual systems exist and the problems arise when we observe them. It is possible to argue that quantum theory says nothing about such individual systems but, even if this is true, the problems do not go away." In rather similar fashion, though Landsberg and Home [224] say of the ensemble interpretation that "if it is accepted certain difficulties fall away", they add that the question whether it is "the best available interpretation", even whether it is "tenable" is "a different one".

Let us now ask this question. In fact, though we have said that (5.5) may be used to calculate relative frequencies for the ensemble, the question remains: does it actually represent the ensemble? (After all, it might be said, it is acknowledged that quantum theory is good at calculating things; the question of interpretation must make a bridge between the elements of the theory, and "how things actually are".)

The most incisive answer has been given by Sudbery [91]. While giving a conference paper on continuous observation and decay in quantum theory, he was challenged by Isham [91, p. 70] to the effect that "everyone knows that a careful treatment of quantum mechanics must be phrased in terms of ensembles". Sudbery answered as follows [91, p. 81]. First, he cleared up any misunderstanding that an ensemble approach was essentially "empirical", dealing with finite numbers of "real" systems. If so, he said, nobody would believe predictions from it, because of the possibility of statistical fluctuations. "A finite ensemble, in fact, constitutes an individual system, and quantum mechanics is not supposed to make predictions about individual systems. So an ensemble must be an infinite affair. But in that case it has no physical reality: it is merely an imaginary picture used as an aid in thinking about the individual system."

(This part of the argument parallels comments of Lucas [46] on the relative frequency view of probability quoted in section 2.6. Let us discuss the statistics a little more explicitly. If a set of indentical measurements of an observable are made on an ensemble in a pure state (not an eigenstate of the observable in question), the eigenvalues occur in a random sequence. If the mean value of the results is $\bar{A}_{N}$, and the quantum mechanical expectation value is $\bar{A}$, the Central Limit Theorem of statistics $[225,226]$ implies that, if such measurements are repeated over many samples of $N$ systems, the various $\bar{A}_{N}$ values will exhibit a normal distribution with mean value $A$ and variance $\sigma_{N}=\sigma / N^{1 / 2}$ (where $\sigma^{2}=\overline{A^{2}}-\langle\bar{A}\rangle^{2}$ ). Thus as long as $N$ is finite, the probability of getting a value of $\bar{A}_{N}$ differing from $\bar{A}$ by any given amount remains nonzero. Just as quantum theory makes no predictions about individual systems, it can make no predictions about finite ensembles. The ensemble used in an ensemble interpretation must therefore be infinite, and so has no empirical significance, being merely an imaginary picture.)

Sudbery then discussed an infinite ensemble: "To say of an individual system that it belongs to an ensemble described by a state vector $c_{1}\left|\psi_{1}\right\rangle+c_{2}\left|\psi_{2}\right\rangle$, of which a proportion $\left|c_{i}\right|^{2}$ exhibits certain characteristics, is to make precisely the same statement as that the system itself is in that state, and the probability that it will exhibit these characteristics is $\left|c_{i}\right|^{2}$." And this assumes a projection postulate; Sudbery says: "The attempt to avoid the projection postulate is equally nugatory... The operational rules of the ensemble interpretation force the usual projection postulate upon it." The point is that the postulate of the interpretation "that a proportion $\left|c_{i}\right|^{2}$ of the systems in the ensemble show certain characteristics requires that it is possible to distinguish some members of the ensemble from others; the ensemble has become inhomogeneous. Before the measurement, on the other hand, there was no difference between the members of the ensemble: this is the significance of describing the ensemble by a pure state. The description of the ensemble after the measurement ought to reflect the fact that it is composed of discernible subensembles: the ensemble should therefore be assigned a mixed state." Its statistical operator should be of the form 


$$
\rho=\sum_{i}\left|c_{i}(t)\right|^{2}\left|\psi_{i}\right\rangle\left\langle\psi_{i}\right|
$$

with, in general, more than one of the $c_{i}$ nonzero.

This result, Sudbery suggests, is inevitable. "[T]he Schrödinger equation is causal and cannot by itself cater for the unpredictability inherent in quantum mechanics. Some extra process such as is provided by the projection postulate is necessary to describe this unpredictability: interpretations like the many-worlds interpretation which try to do without it are attempts to run away from indeterminism."

Sudbery's argument is so important that we rephrase it, particularly contrasting the argument, which is, of course, for the minimal ensemble, with the argument for PIVs. (We feel indeed that the creeping-in of unstated implicit PIVs has very much muddied the water in this area.) With PIVs, (5.4) gives us an ensemble of systems, each of which has a value of $R$, the relative frequencies being $|\langle r \mid \psi\rangle|^{2}$. Still with PIVs, (5.5) gives an ensemble of coupled systems, in each of which the pointer has a position of $\alpha_{r}$, the relative frequencies being the same.

Without PIVs, (5.4) represents an ensemble of systems, each of which is in a linear combination of states $|\mathrm{I} ; r\rangle$, each with relative amplitude $\langle r \mid \psi\rangle$. And (5.5) represents an ensemble of coupled systems, each of which is in a linear combination of states $\left|\mathrm{I} ; \phi_{r}\right\rangle\left|\mathrm{II} ; \alpha_{r}\right\rangle$ again with amplitude $\langle r \mid \psi\rangle$. This certainly does not represent an ensemble on which a measurement has been performed, not, at least, till the collapse on which Sudbery insists. Sudbery indeed says [91, p. 70], "you can, I think, translate everything into the language of ensembles if that makes your mind more comfortable", and the final words of his paper are: "This is part of a general programme of showing that all interpretations of quantum mechanics are saying the same thing in different ways."

To take a specific example, let us consider a Stern-Gerlach apparatus dividing an initial beam of spin-1/2 particles with $S_{x}=1 / 2$ into two beams with $S_{z}= \pm 1 / 2$. Each beam is detected. A PIV theory can, of course, explain why each spin, with its initial value of $S_{z}$, goes along a particular beam. For it, a final state function of the form $(1 / \sqrt{2})\left(\chi_{+} A_{+}+\chi_{-} A_{-}\right)$, where $A_{+}$and $A_{-}$are states of a measuring apparatus, $\chi_{+}$and $\chi_{-}$of spin, does mean that half of the coupled systems correspond to $S_{z}=1 / 2$, and half to $S_{z}=-1 / 2$. For a minimal ensemble interpretation, the state vector says only that each coupled system is in a linear combination of the two states. It only "solves" the measurement problem inasmuch as it says that the ensemble average of $S_{z}$ is equal to the expectation value of $S_{z}$ for the state function above, that is, zero.

To show explicitly how confusion can arise, we note the statement of Gibbons [2, p. 77], discussing the minimal ensemble interpretation. He says: "A measurement of electron spin (for example) will then simply divide an ensemble of electrons into two subensembles, one of spin 'up' electrons and the other of spin 'down', rather than involve a collapse of anything." The lack of collapse stressed suggests that Gibbins is being seduced not just to a PIV picture, but to one in which subensembles with state vectors $\chi_{+}$and $\chi_{-}$are somehow subsumed into the original ensemble $(1 / \sqrt{2})\left(\chi_{+}+\chi_{-}\right)$.

Another interesting analysis is that of Scully et al. [227]. These authors also discuss a Stern-Gerlach apparatus for molecules of spin-1/2, but in the Wigner variation in which the beams are recombined. A detector atom with spin-1/2 may be included near one beam of the device; it is sensitive to the passage of a molecule along that beam. In the absence of the detector atom, the recombined beam has precisely the same form as the initial one-a pure state with an idempotent density matrix containing off-diagonal, as well as diagonal, elements.

When the detector atom is present, however, the interaction may be written as 


$$
(1 / \sqrt{2})\left(\chi_{+}+\chi_{-}\right) ; A_{-} \rightarrow(1 / \sqrt{2})\left(\chi_{+} A_{+}+\chi_{-} A_{-}\right),
$$

where $A_{+}$and $A_{-}$are now the spin states of the atom. The complete $4 \times 4$ density matrix still has off-diagonal elements and is still idempotent. This is natural because the mechanism of Scully et al. follows precisely the Schrödinger equation, so the final state, like the initial state, is pure.

If, however, one restricts attention to the molecular spin, $\chi$, and hence to the reduced $2 \times 2$ density matrix, it is diagonal (in a representation in which the $z$-component of spin is diagonal) and nonidempotent. The state of the beam will appear to be mixed. There can be no interference between the $\chi_{+}$and $\chi_{-}$states because each is coupled to a different member of a pair of orthogonal $A$-states. (This refers, of course, to subsequent measurements of spin only, not to measurements of joint properties of spin and atom.)

The interaction of (5.7), then, mimics many of the aspects of a collapse, and Scully et al. refer to it as a state reduction. (See, also, the discussion of the "improper mixture" in section 3.2.3.) While they are not explicitly supporting an ensemble position, their own position is analogous because they are claiming that collapse, or at least the results of collapse, may be simulated directly from the Schrödinger equation. Just like the ensemble position, they end up with a superposition of states. While this does indeed behave like a mixture if one restricts oneself to the molecular beam only, it is essential for genuine collapse that the $\chi_{+} A_{+}$and $\chi_{-} A_{-}$are in the form of a mixture, not a superposition. The argument could be pushed further by saying that, in a real measurement, the atomic states, $A$, are replaced by the states of a macroscopic detector, so the coupled terms in the superposition cannot interfere.

Other arguments have been put forward claiming to show that the superposition is equivalent to, or indistinguishable from, the mixture one rather wishes it was. Daneri, Loinger and Prosperi [228] put forward an elaborate theory of measurement in which the microscopic part of the process triggers an ergodic amplification in the macroscopic measuring apparatus. However, Jauch, Wigner and Yanase [229] pointed out that the result is still a superposition of macroscopic states, not a genuine mixture. See also the discussion of Jammer [4, pp. 491-493] and references given there.

Another discussion is given by Maxwell [230], who says; "[Ensemble interpretations] can, however, overcome this difficulty, since this viewpoint is not in fact committed to the thesis that the state of the ensemble $E$ somehow mysteriously evolves from a pure state to a mixture... The ensemble $E$ is at time $t$ in the pure state $\psi_{t}$, and not in the mixed state $w_{1} \phi_{1}+w_{2} \phi_{2}$. However the experimental setup is so designed that no measurement can be performed on the particles which would reveal that the state of $E$ is $\psi_{r}$ rather than the mixture. In other words, the experimental setup is so designed that all measurements performed on systems belonging to $E_{1}$ will be in accordance with the strictly false assumption that the state of $E_{1}$ is $\phi_{1}$. According to the [ensemble interpretation], then, there are, strictly speaking, no ensembles with pure states. Rather, in preparing a pure state we so arrange our experimental setups that the (false) assumption that the given ensemble is in a pure state cannot give any false predictions. A preparation device is precisely a device which physically excludes the possibility of measurements which would reveal that the relevant ensemble is not in the desired pure state."

Leggett $[132$, p. 85] is highly critical of this type of argument. He describes an ensemble interpretation as "the most conservative" of "those 'solutions' of the quantum measurement problem which accept the universal application of the quantum formalism". In this interpretation "the formalism is merely a description of our information about the system in question, or more precisely about the ensemble from which it is drawn, and says nothing about the actual state of any particular object, even at the macroscopic level ... [T] he description of the final state of the 'universe' after a measurement has 
been made merely gives us statistical information about the distribution of results obtained in a long series of trials using identical pieces of apparatus and identical initial conditions, and nothing more; the question 'In what macroscopic state is this particular apparatus on this particular occasion?' is one which simply does not lie within the competence of quantum mechanics to answer... This interpretation, while no doubt internally self-consistent, has the very unpleasant feature that under certain conditions, it makes it impossible, even in principle, to give a quantum mechanical description of the state of a particular macroscopic object."

- To the argument that the pure state has the same consequences as the mixture, Leggett retorts that this is "no solution at all... [I]t only looks plausible because of a fundamental ambiguity in the interpretation of the density-matrix formalism (the confusion between proper and improper mixtures [74]) ... [T] level the universe (or the macroscopic apparatus) behaves as if it were in a definite macroscopic state, but whether it is in such a state."

\subsection{Duane's theory}

As shown in section 4.4, Duane's theory [42] is called upon to explain diffraction and interference phenomena by those believing in trajectories for individual particles, which include supporters of PIV ensembles $[3 ; 41 ; 65$, p. 19], though not, of course, those of minimal ensemble interpretations. In Duane's theory, the particle is not considered spread out in space, but the crystal or two-slit apparatus is, and reacts as a solid body, providing a change in momentum $\Delta p=h / l$, where $l$ is an interplanar or inter-slit distance.

In a recent version of the two-slit experiment [231,232], an electron microscope or "electron biprism" has been used to produce the interference pattern. This consists essentially of a conducting wire to which a positive or negative potential is applied to modify the trajectories of electrons passing near, so "the splitting and superposition of the electron beam is achieved by macroscopic fields without any interaction of the electrons with the material" [231].

Rosa [233] has argued that the Duane scheme just cannot work in this case: "[O]ne can clearly realize the difficulties in applying the hypothetical account for an idealized screen with diaphragms to the actual apparatus ... [A]ll the pieces of the assemblage ... should have to react as a single complex entity, so as to exchange selective momenta with incident electrons. Moreover the fringe spacing depends on the slit separation, which, in the actual case, is purely virtual and is a function of the potential applied to the wire. Therefore it seems very hard to interpret the observations in a mechanical fashion." Maxwell too [230] has made criticisms of the Duane idea.

\subsection{General discussion of ensemble interpretations}

Having built up a particular analysis of ensemble interpretations so far in section 5, let us now turn to a study of the literature, and report a range of opinions. We start with a number of authors who have commented fairly widely in the area of quantum interpretation.

Rayski $[234,235]$ has commented specifically on the ensemble interpretation, of which he is a great opponent, in the course of general stimulating discussion on this topic. His general view is that "the 'state' or the 'wave function' is not an observable but is a piece of information. One cannot 'measure an information' but one can get some information by measuring something else... The wave function represents the state of our knowledge about the system, achieved in a complete measurement 
performed at a single instant" [234]. Against Ballentine's comments on such an interpretation, reported in section 3.1, Rayski responds, "such an interpretation is not 'subjective' because it is by no means knowledge about a human mind, but knowledge (or information) about the physical system!" "Such an understanding...", Rayski says [234] "resolves immediately all the difficulties connected with the 'reduction of the state vector'."

Rayski suggests [235] that very many physicists do believe that quantum mechanics refers to ensembles, considers they are arguing from relative-frequency ideas on probability, but concludes: "This is just the point where people are absolutely wrong", and puts forward some simple probabilistic arguments to attempt to make his case. "[P]robabilistic predictions", he concludes, "do apply to single events." He considers [234]: "Contrary to the widespread opinion that - in contradistinction to the classical theory of particles - quantum mechanics is a theory of a statistical character, whose predictions are meaningful only for ensembles, we claim that the epistemological situation is just the opposite. Quantum mechanics constitutes the first example of a theory dealing successfully with single microobjects, whereas classical mechanics is a statistical theory because it deals only with expectation values, i.e., with averages over quantum phenomena."

What was "perhaps the main reason for confusion" [235] was that "many authors . . mixed up two notions: the concept of measuring of observables applying to single physical systems... with an estimation of a wave function" which "cannot be measured but . . . may be estimated statistically [with an ensemble]".

Rayski does reach some conclusions analogous to those of some ensemble theorists, though. From his idea of a "probabilistic interpretation symmetric with respect to time reversal" [234], he is able to make a "conjecture that every observable always possesses some value" [235] (an argument that he claims is not in conflict with Bell), and he suggests that this argument "explains satisfactorily" EPR [234].

Rayski [235] strongly supports an argument of Bohm and Bub [236]. These authors state that the postulates of quantum theory "incorporate the notion of probability into the theory in an inherent way. Yet it is not immediately clear how the ensembles, to which these probabilities refer are formed and what their individual elements are. For the very terminology of quantum mechanics contains an unusual and significant feature, in that what is called the physical state of an individual quantum mechanical system is assumed to manifest itself only in an ensemble of systems. Because it is indeed mysterious for an individual system thus to manifest itself in an ensemble, there is a tendency among physicists tacitly to avoid the problem by interpreting the wave function as referring directly to an ensemble rather than to an individual system." Bohm and Bub claim to show that "[t]his interpretation is . . untenable", by "analysis of a simple hypothetical experiment", a two-slit diffraction experiment with electrons, a movie camera replacing the photographic plate. Electrons may have wave functions of similar shape, but they are "different in their times of going through the slit system". The conclusion drawn is that "the wave function refers primarily to an individual electron and the statistical ensemble refers to a set of electrons having different wave functions of similar shapes". This is a preamble to a paper in which a hidden variable theory is developed.

Peres has written extensively on the interpretation of quantum theory [237-239]. Here we concentrate on his views on ensembles. At first sight they appear to have undergone some change. In 1974 [237], he wrote: "[T]he epistemological difficulties of quantum theory are due to the incorrect application of the notion of 'state' to a single physical system ... This is quite analogous to the fact that a gas has a temperature and pressure, but a single atom in that gas has neither temperature nor pressure ... [A]ny attempt... to apply to individual systems the notion of state which is valid for 
ensembles leads to paradoxes and inconsistencies." This is clearly very close to Ballentine [3], who Peres explicitly acknowledges.

By 1984 his views [238] were at least expressed differently. He distinguishes between two concepts regarding ensembles. The first, proposed by Einstein, and "codified by von Neumann", considers $\psi$ as "an objective attribute of a real ensemble" which is not necessarily infinite; this idea is "not satisfactory, or, at least, is not always applicable, because physical systems can be observed one at a time, not only as members of statistical ensembles". This concept must be distinguished from that of Ballentine, who defines $\psi$ as a "conceptual (infinite) set of all single electrons which have been subjected to some state preparation procedure". Peres supports this, and repeats that "[a] single physical system has no state". However, he adds: "This approach was also advocated by Stapp who called it the Copenhagen interpretation" picking out the remarks of Stapp [88] that $\psi$ represents "[t]he specifications on the manner of preparation of the physical system" and that "[t]he wavefunctions are merely abstract symbolic devices". Peres suggests: "There is no real conflict between Ballentine and Stapp, except that one of them [Ballentine] calls Copenhagen interpretation what the other [Stapp] considers as precisely the opposite of the Copenhagen interpretation." (See section 3.1.) Since Stapp says that "[w]ave functions are associated with the study ... of finite systems" and "[q]uantum theory provides for a complete scientific account of atomic phenomena", neither of which statements sounds very close to Ballentine, it must be concluded that, for Peres, what is most important in both Ballentine and Stapp may be what they are opposed to, the von Neumann idea.

Peres does agree with both that $\psi$ is an attribute of a procedure. In fact, "If we associate $\psi$ with physical events, rather than physical systems, there can be no paradoxes due to the 'collapse of the wave function'. There is no collapse, because there is no interpolating wave function giving the 'state of the system' between measurements." Here any ensemble idea does not appear central. In ref. [239], the results of collapse are obtained by relative phases being "blurred", rather than being avoided by a more straightforward ensemble idea.

Maxwell has also written much concerning ensemble interpretations in connection with his own ideas, and here we concentrate on a pair of his earlier papers $[230,240]$. Anxious about measurement being considered fundamental, he wishes to develop a micro realistic version of quantum theory [240]. He considers that "the primary reason for the abandonment of micro realism" was that "one could ... sidestep the awkward wave-particle duality". He says "the wave aspects of micro phenomena always arise as statistical effects", and "corresponding to two different interpretations of probability, there are two ways in which we may seek to resolve the wave-particle duality problem which do not abandon micro realism".

The first approach, which corresponds to our PIV idea "takes for granted the frequency interpretation of probability" [here Maxwell differs somewhat from our own comments above]; "individual micro systems ... are essentially particles, with definite positions and momenta at each instant, and definite trajectories in space-time". This requires "two quite different notions of state". The "classical state" corresponds to the "actual physical state... specified in terms of the instantaneous position and momentum". The "quantum mechanical state" is specified in terms of the state vector (for a pure state). A micro system in a definite classical state may be in two quite different quantum states, as it may be in two different ensembles.

Maxwell attributes this approach to Landé and Ballentine, but also to Popper, despite the fact that the latter has, for a considerable period, adopted not a frequency theory but a propensity theory (section 2.5.4). However, since Popper relates propensities to experimental setups, not to individual micro systems, Maxwell considers his approach "in all essentials in agreement with Ballentine's 
viewpoint". In particular, "a micro system can only be said to have a quantum mechanical state insofar as it is considered to be a member of an ensemble of micro systems".

This is, Maxwell says, in great contrast to his own notion of propensity, which is a "physical property" and "constitutes a rather natural probabilistic generalization of our ordinary notion of a physical property - namely, of a (deterministic) dispositional property". The propensity viewpoint requires "a potential ensemble" and thus "is ... parasitic on the frequency interpretation of probability". Maxwell considers his idea, though, superior to that of Popper, which "is a macro property, being ... a property of experimental setups" and thus "of no use ... in developing a micro realistic version of QM". Where the traditional approach requires a collapse of wavefunction, Maxwell's requires a "localization" at which the propensity becomes "actualized", a process Maxwell studies in detail [230].

Maxwell [230] also gives a detailed criticism of the "frequency interpretation" (though he says that he would like this approach, as well as his own, to be pursued). He says that "the [frequency] theory cannot enable us to understand why systems evolve in the way in which they do, on the individual level". He believes Duane's argument (sections 4.4 and 5.5) unsuccessful, and even if it did work, considers it supportive of his own ideas, rather than those of Landé and his followers. He thinks that the argument may work for position and momentum, but scarcely for angular momentum or spin, and that any micro relatistic program is "utterly sabotaged" by the fact that measured values bear no relation to premeasurement values. He considers that the view requires trajectories, but regards this as $\mathrm{ad}$ hoc, and is dubious that it could work in any case. For Schrödinger's cat, he requires trajectories again, so that a cat is not "dead at one moment ... and alive at a later moment". (See section 6.2.) Finally he believes the approach requires the notion of classical state, and thus reference to macroscopic objects. Thus he prefers his own propensity view.

Maxwell believes there is a possibility of an experimental test between the two approaches in the field of radioactive decay. On the propensity theory, a negative measurement requires a localization inside the nucleus, which will affect the decay rate, while the frequency theory, he says, does not. (See also section 6.3.)

Selleri and Tarozzi [241] raise "two major objections" against the statistical interpretation. The first is the idea of wave-particle duality which they consider to have been "shown in an extremely convincing way to be a true property of simple quantum systems"; they particularly mention the experiments of "neutron interference with itself" of Rauch et al. [242].

Their second point concerns the fact that "if a measurement is to provide true knowledge of the system when it is left unperturbed", the state of the system "has to be" the eigenstate corresponding to the results of the measurement. "How it is possible to maintain this fact as true without talking about some kind of reduction of the initial state vector", they conclude, "has never been understood by the present authors".

An interesting approach to quantum measurement has been developed by Wan [243] (and later extended by Bub [244]). This is based on the use of superselection rules. The Hilbert space $H$ may be decomposed into a direct sum of mutually orthogonal subspaces, or superselection sectors, $H_{n}$, and if $\phi_{n}$ and $\phi_{m}$ are in different sectors, and $A$ is a bounded observable, $\left(\phi_{n}, A \phi_{m}\right)=0$. Vectors belonging to different sectors are said to be disjoint, and vectors in $H$ can represent mixtures of disjoint states. This allows a transition from a pure state to a mixture, and vice versa. With suitable definition of the measuring device, Wan reports that "the von Neumann projection postulate comes out as a result of our measurement model". He also says, "all this tends to support the statistical interpretation of quantum mechanics" (citing our ref. [3]). 
Pavičić [245] has argued that a difference between "Copenhagen" and "statistical" interpretations may be formulated within the quantum formalism. Previously, he suggests, "it had been taken for granted that a difference, such as those claimed by Ballentine or Park, could only be formulated outside the formalism". With reference to the comment of Jammer mentioned in section 5.3, we remark that Pavičic does indeed rely on "a difference between the frequency and the appropriate probability... which does not vanish as the number of experiments approaches infinity". The difference is written as $g(p)$ where $p$ is the probability. He considers a Bernoulli sequence of trials, and reports, "the values of the frequency never cluster strictly at $p$ but only around $p$ ". An example is then analysed explicitly, that of preparation and measurement by a pair of Stern-Gerlach devices. Of particular interest is the case of field alignment, $p=1$. Since $\left\{N_{+}(1, N), N\right\} \rightarrow 1$, when $p \rightarrow 1$ and $N \rightarrow \infty$, "we are tempted to expect, says Pavičić, that $N_{+}(1, N)=1$. However, if we insist on this, then $g(1)=0, g(p)$ must be discontinuous at $p=1$, and "we cannot but assume that nature differentiates open intervals from closed ones, i.e., distinguishes two infinitely close points". This, then, is the Copenhagen position. The alternative is to take $g(p)$ continuous at $p=1$, and hence nonzero, and then, in fact, as $N \rightarrow \infty,\left\{N_{+}(p, N), N\right\} \rightarrow 0$, even if $p=1$, and we may allow "only a bare statistical meaning to quantum probabilities" even in this case. This is the statistical interpretation according to Pavičic, who concedes that finding an experiment to differentiate between the interpretations may be tricky.

Ghose et al. [246] have suggested a dynamic variant on the double-slit experiment. They base their ideas on the work of Tonomura et al. [247], who have demonstrated single electron interference, and have shown that sending a large number of electrons through the double slit in one shot produces exactly the same interference pattern as sending them through one by one. Ghose et al. quote Rauch's remark [248] that these experiments show that a wave function "describes an individual particle of a certain ensemble" because "there is always only one particle at a time inside the interferometer, but nevertheless one needs many particles for an interference pattern".

In the dynamic variant, a shutter is introduced which can periodically (or randomly) open either or both slits. In a first procedure (A), $N$ electrons in the same state $\psi_{0}$ are sent through one at a time with time intervals large compared to $T$, the period of the shutter. In a second (B), a single bunch is sent through in a time short compared with $T$. In procedure $A$, one expects to see three overlapping patterns, corresponding to $\psi_{1}$ (slit 1 open), $\psi_{2}$, and $a_{1} \psi_{1}+a_{2} \psi_{2}$. In procedure $\mathrm{B}$, one expects only one pattern, either $\psi_{1}, \psi_{2}$, or $a_{1} \psi_{1}+a_{2} \psi_{2}$.

If one adopts an ensemble interpretation, the authors suggest that, with procedure A, "one would have to admit that although the individual members of an ensemble are well separated in their times of origin and detection, their behaviour... shows a strange correlation that may appear 'nonlocal' in time". They say: "There is no such peculiarity if one adopts [an orthodox position]".

Fitchard [249] has proposed an experimental test between Copenhagen and ensemble interpretations. Specifically his experiment tests what he takes to be two main planks of the Copenhagen interpretation - wave packet reduction, and the uncertainty principle, considered as meaningful for simultaneous measurements of position and momentum. The proposed method uses a time-of-flight measurement of momentum, and a coincidence measurement of position. A beam of electrons strikes an atomic or nuclear system with slightly more than threshold energy for a specified transition; it is scattered into a first set of slits. The excited system decays, emitting a photon towards a second set of slits. "By changing the diameter of the first set of slits and the time of flight..., the measurement uncertainty in the electron's initial momentum can be varied. Changing the width of the second set of slits varies the measurement uncertainty in the initial position of the electron." Fitchard argues that the coincidence rate between photons and electrons will depend crucially on whether a collapse takes place 
when the photon is observed. Without collapse, it is always unity, but with a collapse it depends on the value of the product $\Delta x \Delta p$ allowed by the theory, being around 0.3 if the product is $\hbar / 2$. Thus the proposed experiment also tests what Fitchard calls the "measurement-uncertaintly principle".

Scheer and Schmidt [250] have suggested a similar experiment with thermal neutrons, while Matthys and Jaynes [251] have been able to control the phase of a laser amplifier with as few as five photons, thus approaching the uncertainty principle limit of $\Delta n \Delta \phi \sim 1 / 2$.

Lastly we turn to a suggested importance for ensemble ideas in molecular structure. It was mentioned in section 1 that, in a period after the Second World War, ensemble interpretations were favoured in Soviet Russia for ideological reasons. Similarly, Brush [252, p. 426] mentions that valence bond theory was attacked (and preference given to molecular orbital methods), because its concept of resonance was "tainted with idealism because it describes molecular structure in terms of forms that were physically impossible but had only a theoretical existence". (Fortunately, Brush adds, "Russian theoretical chemists could do their calculations however they thought best as long as they did not use the word 'resonance'".)

There perhaps remains in parts of the (not necessarily Russian) chemical literature a feeling that an ensemble interpretation somehow allows different molecules in an ensemble to have different "physically meaningful states", although the wave function is a linear combination of these. Discussion is never explicit, but a number of references are given for consideration [253-256]. Jørgenson [253], for example, refers to the suggestion of Ballentine that "quantum mechanics is only applicable to reproducible small systems with assembly properties". There seems a suggestion of sharing properties across a real physical ensemble, rather than use of an infinite "conceptual" ensemble. We remain unconvinced that discussion of such interpretational matters has any relevance to calculation of molecular structure.

\subsection{Unsharp joint measurements}

Nearly all of this review apart from this section follows refs. [3, 22, 38, 241], and much of ref. [4] in being based almost entirely on von Neumann's development of quantum theory. In recent years axiomatic methods have been developed by Mackey [257], Piron [258], Jauch [259], Mittelstaedt [260], Ludwig [181, 261], and Lewis and Davies [182, 183]. Though any full mathematical treatment must be outside the scope of this review, we give here an account of how one of the methods tackles one of our central themes - the possibility of joint measurements of position and momentum.

This problem has been studied extensively by two groups: Busch and Lahti $[179,262,263]$ and de Muynck and coworkers [264,265]. Here we largely follow ref. [263], and refer to it as BL. This reference begins by contrasting three views on the problem. The first, attributed to Heisenberg, and which BL say "belongs to the conceptual core of the so-called Copenhagen interpretation", is called here the "usual view" (though BL call it "standard" - not to be associated with "standard frame" below). It says that "complementary physical quantities . . can simultaneously be measured only to the accuracy allowed by the ... uncertainty relations". The "first extreme view" which is "compatible with the intuitive notion of complementary physical quantities as developed by Pauli and Bohr" says that "complementary physical quantities cannot simultaneously be measured at all". In this context, BL quote the remarks of Suppes [266]: "The conclusion that momentum and position are not simultaneously measurable at all does not follow from the Heisenberg relation but from the more fundamental results about the absence of any genuine joint distribution." The "second extreme view" attributed to such workers as Margenau, Park and Ballentine claims that "the uncertainty principle is irrelevant to 
the problem of simultaneous measurability of complementary physical quantities", but that "such quantities can simultaneously be measured within any accuracy".

BL use the "convexity frame" $[182,183]$ for their analysis. The definition of a convex set is that, if $\beta_{1}$ and $\beta_{2}$ are in the set, then $\lambda \beta_{1}+(1-\lambda) \beta_{2}$ is also in the set, where $0<\lambda<1$. For an extreme point, $\beta$ of the set, the relationship $\phi=\lambda \beta_{1}+(1-\lambda) \beta_{2}$ implies that $\beta_{1}=\beta_{2}=\beta$. This corresponds to a pure state in quantum theory, while a nonextreme point corresponds to a mixed state.

The convexity scheme also contains the following ingredients. An operation or state transformation, $\phi$, when performed on a system, changes a given initial state into a well-defined final state, and is represented mathematically be a linear mapping in which the norm either decreases, or remains constant. The effect of an operator is defined by $e \phi$ where $e$ gives the norm of any state. An instrument corresponds to an experimental arrangement, and defines a family of operations that may be performed on the system with the instrument. To each instrument there is an observable corresponding to the family of the detectable effects of the operations performed with the instrument.

The Hilbert space may be regarded as a realisation of the convexity scheme, but it is unnecessarily restricted in that its unit vectors correspond to one-dimensional projections, and hence only to the extreme elements of the set, the pure states. It thus considers only observables which are represented by projection-valued measures [182, p. 36], and only operations which give rise to projections as their effects, such as the von Neumann-Lüders operations [95], measurements of the first kind (section 3.2.2). This constitutes what BL call "standard frame" quantum theory, as opposed to the convexity approach which gives the "extended frame".

In this approach, a measurement on a system is described via a transformation on the states of the system, $\alpha_{i} \rightarrow \alpha_{\mathrm{f}}$ where $\alpha_{\mathrm{f}}$ depends, in general, on $\alpha_{\mathrm{i}}$, the result of the measurement, and the measuring instrument used. Any measurement is a measurement of some effect; it is an operation providing (probabilistic) information on the effect.

Any measurement is deterministic; it provides probabilistic information on the effect in the premeasurement state. The statistics of the results of measurement of a complete set of observables allows one to determine the initial state. If there exists a state $\alpha$ such that $e(\phi \alpha)=1$. then $\phi$ is said to be predictable.

A measurement is also preparatory or weakly repeatable if $e\left(\phi^{2} \alpha\right)=e(\phi \alpha)$ for any state $\alpha$; the second operation, $\phi$, has no influence over and above the first. If, in addition, one insists that if $e(\phi \alpha)=e(\alpha)$, then $\phi \alpha=\alpha$ for any state $\alpha$, the measurement is termed strongly repeatable or of the first kind. A preparatory measurement is a predictable measurement.

BL now define complementary observables in the following way. Any two instruments uniquely defining such observables are mutually exclusive in the sense that each of the instruments can provide no information on the other observable. (This goes further than incompatibility, defined by $[A, B] \neq 0$. Incompatible observables may have some common eigenvectors, but not a complete set; complementary observables have no common eigenvectors.) It is then said to be an obvious result that "complementary observables do not admit any joint measurements".

$\mathrm{BL}$ then define, however, another concept, that of probabilistic complementarity. For two observables $A$ and $B$ to be probabilistically complementary, if $(\phi|A| \phi)=1$ for some $\phi$, then $(\phi|B| \phi)<1$. For the standard frame, the notions of complementarity and probabilistic complementarity are equivalent, but in the extended frame, the first implies the second, but not vice versa. It is easy to show that "probabilistic complementary observables do not admit of any predictable joint measurements".

BL now consider the question of joint measurements of $q$ and $p$ in the standard frame. The obstacle is 
the mutual exclusivity of the instruments, but traditionally it is suggested that this may be overcome by discussion of "ambiguities, or inaccuracies" in the definitions or measurements of the observables, to the extent expressed in the uncertainty relations. Intuitively one is inclined to identify the standard deviation $\Delta(A, \alpha)$ of a physical quantity $A$ in state $\alpha$ with "the characteristic accuracy $\delta(A, \alpha)$ in determining the value of the quantity $A$ in the state $\alpha$, or with the inaccuracy in an $A$ measurement performed on the system in the state $\alpha$ ". However BL point out that this is an ad hoc assumption, and illegitimate within standard frame theory. They conclude: "Within the standard Hilbert-space quantum theory, complementary physical quantities, like position and momentum, cannot be simultaneously measured at all. This does not follow from the uncertainty principle, which therein is to be interpreted probabilistically, but rather from the very idea of complementarity."

On the other hand, in the extended frame, it is possible to introduce formally the idea of unsharp variables. The idea comes directly from Heisenberg's idea of a $q$-measurement in his $\gamma$-ray microscope being inherently inaccurate - the value itself being unsharp or "fuzzy". Formally an apparatus measures a "fuzzy" value set $x+X$ with $-\delta / 2<x<\delta / 2$ rather than a value set $X$. A probability density function, $f(x)$, is introduced to describe either the unsharpness involved in the measurement, or the ambiguity in the definition of the observable. We may now identify $\delta(A, \alpha)$, not with $\Delta(A, \alpha)$ as above, but with $\Delta f$, the standard deviation of $f$. Also the basic probability measures of the theory are allowed a double role - first, to the possible values of the observable, second to ambiguities or inaccuracies in measurement. "It is just this feature of the extended frame which makes it possible that the uncertainty relations may have something to do with the imprecisions of measurement as stated in the [usual] view."

Indeed, in the extended frame, BL are able to demonstrate: "Position and momentum observables $q$ and $p$ can simultaneously be measured with measuring accuracies which may or may not obey the uncertainty relations. In particular, uncertainty relations do express neither necessary nor sufficient conditions for their joint measurability." The sting in the tail is: "None of the possible joint measurements of position and momentum observables is, however, predictable or preparatory."

In the above general statement, the uncertainty relations play no part. One can however recover a little of the determinative and preparatory features of sharp measurements if one does accept them. One may then speak of a joint measurement of $p$ and $q$, or of a quasipreparatory measurement, which prepares objective but unsharp position and/or momentum values. "It is in this sense that the uncertainty relations may be interpreted to show up an aspect of complementarity: instruments corresponding to complementary observables exclude each other not only in the sense described [above] but also in that they disturb each other (if simultaneously applied) to the extent given by the uncertainty relation." Thus: "By introducing the extended frame certain formal restrictions contained in the language of the standard frame can be weakened... [L]anguage becomes more similar to classical language in this respect as one may talk about joint measurements of (probabilistically) complementary observables. However, there is a price to be paid which consists in a loss of informational content of the measuring statements... Thus the nonclassical nature of the quantum language remains preserved."

BL conclude by analysing experiments of Park and Margenau [100], and Ballentine [3] which claim to provide joint measurements of position and momentum of arguable accuracy. BL suggest that "the time-of-flight method fails to serve as an example of the second extreme view [above]", and that, in Ballentine's slit experiment, "the assignment of some calculated momentum value is ad hoc and has nothing to do with a momentum measurement". 


\section{Application to specific problems}

\subsection{EPR problem}

At the end of section 4 we mentioned Ballentine's remark that it is only through analysis of special problems that the weakness of the orthodox position is demonstrated, so this section of the paper is one of the most important. There is indeed a common belief that ensemble interpretations do remove all the difficulties. To the suggestion that "the difficulties associated with measurements in quantum mechanics [might be used] to explain paranormal phenomena", for instance, Taylor and Balonovski [267] have no hesitation in replying that "the standard statistical interpretation of quantum mechanics has none of the claimed paradoxes".

Let us start with EPR (all discussion being of Bohm's version [146]), and review Ballentine's solution to the problem [3]. Actually he provides two. The first, not particularly explicit, essentially follows EPR themselves in regarding the problem as establishing the existence of PIVs. It is described [3, p. 363] as "unreasonable, essentially solipsist", to "[deny] the reality of both $\sigma_{1 z}$ and $\sigma_{2 z}$ until the measurement has actually been performed". With PIVs, it is indeed quite clear that the EPR problem is no problem at all. (We would stress, of course, that this refers only to the original Bohm discussion where the fields in the two wings of the experiment are parallel, not to the general case considered by Bell.) All this achieves, of course, is to return us to the argument of sections 5.1 and 5.2 which shows how extremely difficult the establishment of a PIV interpretation must be. Bell's work [21] showed that locality could not be maintained for a class of theory including, but wider than, PIV theories, deterministic and also stochastic hidden-variables theories.

We briefly mention the views of Einstein and Rosen themselves. It was shown in section 4.3 that Einstein's ideas of the ensemble were never made clear. We believe the easiest way to interpret his writing, particularly on the EPR problem itself, is that he supported PIV ideas, and ignored the difficulties. Rosen's views [82, p. 17] were also mentioned in that section. Again it seems easiest to interpret these as based on PIVs. There is certainly a recognition of some of the problems, but we would suggest that a distinction between "coherent ensembles" (for pure states) and "incoherent ensembles" (for classical physics and mixed states) is more a statement of the problem than a solution.

We now turn to Ballentine's second explanation [3, p. 364]. In the ensemble interpretation, the meaning of the state vector

$$
\psi=(1 / \sqrt{2})\left[u_{+}(1) u_{-}(2)-u_{-}(1) u_{+}(2)\right]
$$

(where we suppress time factors), he says, is that "if the experiment is repeated many times we should obtain the result $\ldots \sigma_{1 z}=+1, \sigma_{2 z}=-1$ in about one-half of the cases, and the opposite result in the other half of the cases". We call this statement $X$.

Again the argument will work perfectly well with PIVs. In that case, the state-vector means exactly what Ballentine says. In a minimal ensemble interpretation, though, we should face the fact that each system in the ensemble is in a superposition of the two coupled states. (See section 5.4.) If a measurement of $z$-component of spin is performed in each wing of the apparatus, the formalism will again predict a superposition of $u_{+}(1) u_{-}(2) \psi_{+}(1) \psi_{-}(2)$ and $u_{-}(1) u_{+}(2) \psi_{-}(1) \psi_{+}(2)$, where the $\psi \mathrm{s}$ denote the states of the measuring devices. The combined state is suitably correlated within each term of the superposition, but nevertheless it is a superposition, not a mixture.

Most of those discussing an ensemble approach to EPR have argued in broadly similar terms to 
statement $X$ above (or Ballentine's other argument), with varying amounts of attention paid to the problem of the previous paragraph. Two interesting papers on ensemble interpretations in general, by Ross-Bonney [268] and Fox [269] have not been mentioned until now because their main thrust is towards the problems of this section. Ross-Bonney's approach to EPR is to liken the situation to throwing a die. "If the top face of the die shows a six ... then without looking we know that on the bottom is a one." It is clear that this is explicitly a PIV approach.

Fox [269] essentially takes his results from the correlations of (6.1). Thus if $S_{1 z}$ is found to be $-\hbar / 2, S_{2 z}$ must be $\hbar / 2$. Fox specifically addresses an important point in EPR's argument, that the measurements may be made of $x$-, rather that $z$-components. His previous explanation clearly still holds, since (6.1) is spherically symmetric. Fox says: "Depending upon our choice of measurement made on the particle 1 , and its result, our predictions about the values of the observables of particle 2 are modified, but its state is not." (One feels uneasy about this statement; if predictions are modified, then even if the state vector of the system is unchanged, the state-to be interpreted in the straightforward way as what the system is really like-must surely have changed, but for Fox's statement to be interesting, the latter and not the former must be implied.) On the PIV assumption, Fox appears neutral - at this point anyway (see section 6.3): "[W]hether the possible predictions about the values of different spin components of particle 2 in the two situations described correspond to simultaneous objectively real values ... is another question which is outside the scope of this paper."

Landsberg and Home [224] deduce from their own formalism, "The ensemble-averaged expectation values of the spin variables pertaining to the second particles are not affected by the measurement, or the type of measurement, made on the first particles of the systems of the ensemble."

Newton [120] answers the superposition-mixture dilemma as follows: "It is correct to say that the spin- $1 / 2$ daughters of the decay of a spin- 0 particle are not automatically in mixed states individually... However, if one performs measurements on one of the fragments alone then, for the purpose of . . purely individual measurements, the one-fragment systems are in a normal mixed state. That is what one usually means by asking for a state of [an individual system]." One perhaps feels that at best, as Leggett says (section 4.4) this is suggesting that the superposition may act as if it were a mixture.

Several authors $[259,270]$ have suggested that the EPR "paradox" may easily be resolved by use of the density matrix. Essentially the idea is that of Newton above. The density matrix for the coupled system is, of course, pure, but, considering the $2 \times 2$ reduced density matrix for either spin, and taking the partial trace over the states of the other, an improper mixture is produced, in the terms of d'Espagnat [74] (section 3.2.3). Whitaker and Singh [271, 272] have argued that this resolution relies on the fact that implicitly they are using an ensemble interpretation. The argument is essentially that a nonidempotent density matrix cannot be written down for a single system, only for an ensemble.

Let us now, though, analyse the situation in more directly physical terms. It has been stressed, particularly by Bell [273] that EPR is not a discussion of formalism. It is a physical argument that, if locality is assumed, the perfect (anti-) correlation of the measured values of $S_{z}$ on the two wings of the experiment can only be explained by "properties of some kind . . chosen by the source". This implies PIVs, or, more generally, deterministic hidden variables. (This argument was Bell's starting point in (the second paragraph of) ref. [21], though it has often been missed, as mentioned in note 10 of ref. [273].)

Clearly then, as already remarked, a PIV-ensemble interpretation has no difficulty in explaining the results of the thought experiment. Equally clearly, orthodox interpretations, denied PIVs or any other hidden variables, are forced to take, more or less explicitly, the nonlocality route. (Bohr's own position 
[110] (section 3.3.2) denies nonlocal mechanical disturbance, but allows a measurement on one wing to influence what can be said about the system on the other wing.)

The minimal ensemble interpretation, again without recourse to PIVs or hidden variables, is in the same position as the orthodox interpretation. The results of the measurement only come into existence at the time of the measurements, and again one has to explain the correlations. Judged in this simple context, the verbal manoeuvring and formal analysis of Newton and Fox seem to miss the point.

The challenge of EPR is not just to explain in a formal way how the superposition of (6.1) becomes, or acts as if it becomes, a mixture at a measurement. That is to say, it does not demand a formal explanation for the effects of wave-function collapse; it demands physical understanding of how the experimental results emerge.

It may be argued that this discussion misses the central point of any ensemble interpretation, which is that quantum theory predicts results only for ensembles. What this actually means is that the theory does not attempt to predict for which pairs of events $S_{1 z}$ equals $1 / 2$, and $S_{2 z}$ is $-1 / 2$, and for which the values are reversed. It should not evade the fact that every pair is correlated. (One may bring in d'Espagnat's "element of reality" from section 2.8 if required.) This is the physical challenge of EPR to both orthodox and minimal ensemble interpretations.

We now turn briefly to the work of Gibbins [2, p. 76] who argues that the minimal ensemble interpretation does, in fact, dispose of EPR. "EPR", he says, "does not demonstrate a violation of statistical locality. In other words, the relative frequencies of measurement results on system II cannot be altered by measurements on I."

It should be pointed out that, in this type of experiment, locality requires absence of effect on results in system II, not just of the type of measurement made on I, but also of the results obtained in I [274]. In this case $S_{1 z}$ and $S_{2 z}$ averaged over an ensemble will indeed be individually (almost) zero. On the other hand, $S_{1 z} S_{2 z}$ will be equal to $-\hbar^{2} / 4$ on each measurement, so clearly its average over the ensemble is also nonzero. This demonstrates lack of locality (if, of course, one may not assume hidden variables).

The term "statistical locality" may be examined by extending the analysis to the Bell case where the fields are not aligned, but along directions $z$ and $z^{\prime}$. In this case, $S_{1 z}$ and $S_{2 z}$, will average to zero across the ensemble; $S_{1 z} S_{2 z}$, will vary across the ensemble, but, in general, will not average to zero. This still demonstrates nonlocality. We could, perhaps use the term "statistical locality" here, reserving the term "locality" (or, in fact, its absence) for the case where $z$ is identical to $z$ ', and each value of $S_{1 z} S_{2 z}$ is the same, but this terminology is unnecessary and unhelpful. Locality, or its absence, is invariably deduced from a series of experimental trials, since a single result proves nothing. In the general case, theory too makes predictions only for averages over a number of trials. This is not in any sense a distinct feature of ensemble interpretations.

To conclude this section, we examine a paper by Breitenberger [275]. It is of interest because it specifically criticises Ballentine's discussion [3] of EPR. Though the criticisms (which are, in our view, misconceived) are not centrally related to ensembles, they are generally relevant in the context of the present paper. They relate to Ballentine's initial explanation of the EPR problem.

Breitenberger states that each of $\sigma_{2 z}$ and $\sigma_{2 x}$ is independently an element of reality for the EPR-Bohm problem in the sense that we can predict its value without disturbing the system. He then says, "Since no state vector can provide a value for . . $\sigma_{2 x}$ and $\sigma_{2 z}$, EPR conclude . . that the state vector does not provide a complete description of an individual system."

Breitenberger studies two different interpretations of Ballentine's argument. The first considers two experiments performed, the first measuring $\sigma_{2 z}$ and not disturbing it, the second measuring $\sigma_{2 x}$. Since 
the first measurement will, in general, disturb $\sigma_{2 x}$, the value obtained by the experiment will not in general be the value obtained if $\sigma_{2 x}$ had been measured initially instead of $\sigma_{2 z}$. This it is suggested disproves the notion that $\sigma_{2 x}$ and $\sigma_{2 z}$ are simultaneously elements of reality. This is a simple argument, and almost certainly the interpretation is not what Ballentine intended.

Rather he meant the second possibility which Breitenberger also considers, but dismisses as a "crude fallacy". He paraphrases Ballentine's argument as: "Since it is open to us to make a measurement of the spin component of particle 2 in any direction whatever, it follows ... that any component of the spin of particle 2 must exist in a well-defined form, and therefore that any two components in different directions must exist jointly in a well-defined form."

Breitenberger criticises this argument by remarking: "If I am standing upright, I can raise the right leg, or I can raise the left leg, but it does not follow that I can raise both legs (and still be standing upright)." (He also puts the argument more formally, but we consider his logic flawed by having "or"s where there should be "and"s.) Let us consider the basic idea. In fact, "If $A$ then $B$ " and "if $A$ then $C$ " does imply "If $A$ then ( $B$ and $C$ )" provided $A, B$ and $C$ are truth statements or existence statements. For Breitenberger, $B / C$ are "It is true that I can lift my right/left leg [and (implied) perform no other action]." Ballentine's statements are existence statements, and therefore his argument holds.

The situation is reminiscent of Schrödinger's scholar [212, p. 164], who, on a number of occasions, is asked two questions in varying order. He always answers the first question correctly, though this so tires or confuses him that his second answer is worthless. But, says Schrödinger, the evidence shows that "he knows the answer to both questions".

Our conclusion then is to reject Breitenberger's criticism of Ballentine's statement (as distinct from solution) of the EPR problem.

\subsection{Schrödinger's cat}

In Schrödinger's cat "paradox" [212, p. 157], one writes down a wave function of the form $(1 / \sqrt{2})\left(\phi_{\mathrm{d}} \psi_{\mathrm{d}}+\phi_{\mathrm{s}} \psi_{\mathrm{a}}\right)$, where $\phi_{\mathrm{d}}$ and $\phi_{\mathrm{s}}$ represent "decayed" and "surviving" states for a radioactive atom, and $\psi_{\mathrm{d}}$ and $\psi_{\mathrm{a}}$ "dead" and "alive" states of a cat. The argument then points out that it is inconceivable that a macroscopic object such as a cat could genuinely be represented by such a function. Also, simple von Neumann ideas would suggest that it is only when the box is opened and inspected by an observer that the wavefunction collapses to either $\phi_{\mathrm{d}} \psi_{\mathrm{d}}$, or to $\phi_{\mathrm{s}} \psi_{\mathrm{a}}$ and the cat is actually killed - or not. Again this seems ridiculous.

Let us see how an ensemble interpretation may handle this, starting with a PIV ensemble. In this case, the wave function above represents an ensemble of coupled systems, in half of which the atom has not decayed and the cat is alive, and in the other half, the atom has decayed and the cat is dead. When the experimenter opens the box, all he does is discover which of the sub-ensembles his particular case belongs to.

While such an analysis is pleasing, there are additional questions about a PIV scheme in this case involving decay, over and above the profound difficulties discussed in sections 5.1 and 5.2. Is it inherent in the scheme that a given radioactive atom is programmed to decay at a certain time? The numbers programmed to decay at particular times could give the exponential, or near exponential, overall profile. In other words, time (of decay) would, in many ways, be like a PIV. Another possibility is that, while each atom is, at any time, decayed or not, each cat dead or alive (this just being the PIV idea), the way in which individual atoms would decay at individual times could be probabilistic. Or to take one further step following Maxwell [230], one might even ask whether an atom must "decay" in a 
once-for-all-time sense. While statistically there must be a movement in the direction of decay, the formalism does not seem to require that any individual atom could not be regenerated, individual cats reborn, perhaps several times.

While these questions are interesting, and will reappear in section 6.3 , we do not need to consider them here. To the extent that we restrict ourselves to the contents of the second paragraph of this section, we may say that the PIV interpretation is able to solve the "paradox".

However the interpretation of the same wavefunction under a minimal ensemble interpretation is entirely different. It represents an ensemble of coupled systems, each of which is a superposition of $\phi_{\mathrm{d}} \psi_{\mathrm{d}}$ and $\phi_{\mathrm{s}} \psi_{\mathrm{a}}$, so we have no real solution of the difficulty. (See, again, section 4.4.)

The ensemble theorist who makes the greatest effort to overcoming the dilemma is Ross-Bonney [268]. He starts by saying: "In the statistical interpretation there is no problem. The wave function brutally represents a vast array of similarly placed cats. In any particular experiment about half the cats are dead ..., and about half are alive." He claims though that this state "is not a mixture", and "Nor is there any, other than accidental, reason to suppose this." He says, "[The experimental prediction] is an interpretation of a pure wave function (cat $50 \%$ dead) and not a transition to a mixed state. The wave function develops in time according to Schrödinger's equation, which enables us to calculate mathematical probabilities for the occurrence of certain events, and to make appropriate predictions. Occasionally an event may have probability unity, but usually it will not. We can, in general, using the density-matrix formulation, separate the predictions associated with a pure state from those associated with a mixed state. If for some particular type of measurement we can set up a pure state that predicts certain results to be the same as those of a mixed one, this is not in itself a criticism of the "statistical interpretation'. (Though possibly 'probabilistic interpretation' might be a verbal improvement, less misleading or confusing than the former.) Certainly, in the cat case we can set up a macroscopic situation in which we break the flasks in some suitably designed random way with the right probability to duplicate the results of cat mortality (with similar statistical variations)."

To comment on this briefly, it is certainly true that mathematical probabilities of occurrence of certain events may be predicted from the Schrödinger equation (not from any interpretation of it). The job of an interpretation is to say why or how things happen. The fact that quantum theory may say that some properties of a given mixed state and a given pure state are the same, can in no way entitle one to identify the states, particularly as concerns the crucial point in which they are different - one is mixed and the other pure! (See Leggett's comments quoted in section 4.4.)

Let us follow Ross-Bonney a little further. He makes an analogy with a Stern-Gerlach-Wigner apparatus. The particles travel in the $z$-direction, and have spins polarised in the $x$-direction ("up"). Magnet A, with field in the $y$-direction, separates the particles, and magnet B recombines them. "[The experimenter] then uses a further magnet and counters to show that he has a pure state - particles with spin "up" in the $x$-direction. However, if unknown to him, I sneak some material between A and B so that one of the beams is stopped and the other is not, his results will be that (approximately, of course) $50 \%$ of his particles are spin up and $50 \%$ spin down. He may interpret this as an unpolarized beam - that is, not a pure state. But you and I know that it is polarized in the $y$-direction. It is in a pure state. So also our cats."

It is certainly the case that a Stern-Gerlach apparatus with field in the $x$-direction cannot distinguish an unpolarised beam from a beam polarised in the $y$-direction. This does not mean that they are identical! In the first place, other experiments can distinguish them - notably a Stern-Gerlach with field in the $y$-direction. But even if this were not the case - and in the general measurement problem and the cat case, it will not be, because the elements of the superposition are macroscopic and so cannot 
interfere - one must repeat that a mixture is inherently different from a superposition. The challenge of the ensemble supporter often seems to be to perform operations, theoretical or experimental, on the pure state to demonstrate that it is not a mixture. The fundamental point is that a superposition cannot represent the result of a measurement.

We close this section by noting a recent exchange of views on the Schrödinger cat problem between Loeser [276, 278] and Ballentine [277]. Though it does not specifically relate to ensembles, it is of interest in demonstrating Ballentine's general views on the subject.

\subsection{The quantum Zeno (watched-pot) "paradox"}

The so-called quantum Zeno paradox has been discussed reasonably fully only comparatively recently [279-284], though there were earlier brief mentions, cited in ref. [285]. The effect relies on the fact that quantum theory does not allow radioactive decay to be strictly exponential. Under reasonably wide conditions, it ends as an inverse power law (possibly with oscillations), and, which is important here, begins as a quadratic function of time. (See ref. [286] and references therein.) The "paradox" is the prediction that, under "continuous observation", the radioactive decay will be halted.

It is emphasised that what is meant by "continuous" observation is merely the presence of a collector to detect the decay particle. (The collector should occupy the full solid angle around the radioactive atom to be sure of detection.) It is very difficult to imagine that the mere presence of a detector could affect the decay, let alone quench it, and so the prediction does appear genuinely paradoxical. The point has been stressed because there are analogous effects where an actual physical disturbance causes inhibition of decay via loss of phase coherence [287-289]. Similarly, it has been claimed that recent experiments of Itano et al. [285] demonstrate the quantum Zeno effect, but others [290, 291] have shown that, in order to understand the result, "measurement" does not need to be treated as a concept outside the Schrödinger equation.

One might wish to use the term "quantum Zeno effect" for these interesting, but easily understood experiments. Their existence should not allow one to forget the (thought-) experiments mentioned above, where measurement (even a negative measurement) does play a central role, and the term "quantum Zeno paradox" could be reserved for this case. (On the subject of terminology, Sudbery [91] says that the classical Zeno paradox results from the fact that the equations of motion are second-order in time. Since Schrödinger's equation is first-order in time, Sudbery argues that there can be no quantum Zeno paradox, and he prefers the term "watched-pot paradox". We stick to the slightly more usual term here.)

Let us now describe a simple approach to the quantum Zeno "paradox". When an unstable system has been decaying for a time $t$, we may write a wave function of the form $a_{\mathrm{s}} \phi_{\mathrm{s}}+a_{\mathrm{d}} \phi_{\mathrm{d}}$, where $\phi_{\mathrm{s}}$ and $\phi_{\mathrm{d}}$ represent "surviving" and "decayed" states, and $a_{\mathrm{s}}$ and $a_{\mathrm{d}}$ are appropriate coefficients. Since we are working (throughout) in the $t^{2}$-region of decay, $a_{\mathrm{d}}$ is proportional to $t$, and the probability of decay, $\left|a_{\mathrm{d}}\right|^{2}$, or $P_{\mathrm{d}}$ is proportional to $t^{2}$. The rate of decay, $\mathrm{d} P_{\mathrm{d}} / \mathrm{d} t$ thus increases with time, and this is because of phase coherence between $\phi_{\mathrm{s}}$ and $\phi_{\mathrm{d}}$.

In fact we may write, from Schrödinger's equation [281]

$$
\begin{aligned}
& \dot{a}_{\mathrm{s}}=-(\mathrm{i} / \hbar) V_{\mathrm{sd}} a_{\mathrm{d}} \exp \left[\mathrm{i}\left(E_{\mathrm{s}}-E_{\mathrm{d}}\right) t / \hbar\right], \\
& \dot{a}_{\mathrm{d}}=-(\mathrm{i} / \hbar) V_{\mathrm{ds}} a_{\mathrm{s}} \exp \left[\mathrm{i}\left(E_{\mathrm{d}}-E_{\mathrm{s}}\right) t / \hbar\right],
\end{aligned}
$$


where we are using a simplified two-level system. [Naturally for other purposes, including the change to the (quasi-) exponential part of the decay, the energy level for decayed states would need to be broadened. But this simple perspective is adequate for our purposes.] The elements of $V$ are the off-diagonal elements of the Hamiltonian matrix for the two-level system. So

$$
\ddot{a}_{\mathrm{s}}=-\left(\left|V_{\mathrm{sd}}\right|^{2} / \hbar^{2}\right) a_{\mathrm{s}},
$$

and with $a_{\mathrm{s}}(0)=1, \dot{a}_{\mathrm{s}}(0)=0$, and for small $t$, we obtain

$$
\begin{aligned}
& a_{\mathrm{s}}=1-\left|V_{\mathrm{sd}}\right|^{2} t^{2} / 2 \hbar^{2} . \\
& a_{\mathrm{d}}=-(\mathrm{i} t / \hbar) V_{\mathrm{ds}} \exp \left[\mathrm{i}\left(E_{\mathrm{d}}-E_{\mathrm{s}}\right) t / \hbar\right], \\
& P_{\mathrm{d}}=\left|V_{\mathrm{sd}}\right|^{2} t^{2} / \hbar^{2}=1-P_{\mathrm{s}} .
\end{aligned}
$$

If, however, $a_{\mathrm{d}}$ is reduced towards zero, or subject to random changes in phase, (6.2) shows that any change in $a_{s}$ will be diminished, and in the limit eliminated altogether.

According to a von Neumann treatment, this is precisely what repeated, or, in the limit, continuous measurement will achieve. Let us imagine a measurement at time $t_{0} / n$. Assuming survival at this time, the wave function collapses to $\phi_{s}$, and a new $t^{2}$-region commences. Measurements performed $n$ times at intervals $t_{0} / n$ will give

$$
P_{\mathrm{s}}\left(t_{0}\right)=\left[1-\left(\left|V_{\mathrm{sd}}\right|^{2} t_{0}^{2}\right) /\left(n^{2} \hbar^{2}\right)\right]^{n}=1-\left(\left|V_{\mathrm{sd}}\right|^{2} t_{0}^{2} / n \hbar^{2}\right) \cdots
$$

(for small $t_{0}$ ), and $P_{\mathrm{s}}\left(t_{0}\right)$ tends to 1 as $n$ tends to infinity.

Now let us analyse the same situation according to an ensemble interpretation. A fairly obvious suggestion (e.g., P. Pearle, quoted by Sudbery [91]) is to say that the quantum Zeno problem is a result of collapse, an ensemble interpretation does not require collapse, so we predict no quantum Zeno paradox. This leads to the possibility of a test between orthodox and ensemble interpretations. As already mentioned, Maxwell [230] has suggested just such a test, his "propensity interpretation" being equivalent to the orthodox one in this context.

Yet second thoughts may make us a little suspicious of this argument. Even to talk of experimental test shows us the different nature of this "paradox" from say, EPR. In the latter, the results of the (thought-) experiment are assumed known, the challenge for different interpretations being to explain them in as convincing a way as possible. (Of course this does not apply to Bell's extension to EPR, where the results of quantum theory are deliberately questioned.) The quantum Zeno prediction is from a particular interpretation of quantum theory, which may be challenged by others. Yet again, in the most literal sense of the word "interpretation", different interpretations should not give different predictions, as their only function is to "interpret" or "explain" a known body of theory and experimental fact. This argument can be at most suggestive; it could well be said that different interpretations should agree with theory, experiment and each other in the well-established areas of the subject, but may differ in subtleties not directly and conclusively testable (or not yet tested) by experiment or theory. Yet it is an argument that should at least warn us to be very wary of easy conclusions.

A PIV approach also seems beset with questions, many of the issues raised in section 6.2 being 
relevant. It may be natural in a PIV approach to assume that the decay time of each atom is also fixed in advance; it could then be felt that the measurement could have no effect on the behaviour, and no quantum Zeno process could occur. The assumption of predetermined values is not essential. We may assume the decay to be genuinely probabilistic but again, if we assume this probabilistic behaviour uninfluenced by measurement, the quantum Zeno effect is still excluded.

A stance along these general lines seems to have been adopted by J.R. Fox in an unpublished note of 1983, cited by Ballentine [39], and following on from his paper discussed in section 6.1. Fox appears to be ambivalent about PIVs. He says of the "Statistical Interpretation" that "the state vector describes not a single system but an infinite conceptual ensemble of similarly prepared systems, and carries information about the statistical distribution of (well-defined) values of observation [our emphasis] taken by members of the ensemble"; but adds in a note: "It is not at present claimed that the members of the ensemble have simultaneously well-defined values of all observables, at least not without some extension of the ensemble concept." One can well understand the desire to gain the advantages of the PIV idea, to shrug off the undoubted severe problems (sections 5.1 and 5.2), but one remains unconvinced that this approach can be justified. In connection with our comments above, it is interesting that Fox suggests that each atom "has a (reasonably) well-defined instant of decay".

If one does adopt this approach, it is fairly easy to feel that the process of reduction at a measurement "is not something physical but is a mental act on the part of the observer", and that "[i]f we ... take the view that the state vector describes the physical system in an objective sense, reduction of it cannot occur". Thus the "common sense quantum-mechanical result" is regained: "Quantum systems can change their 'state' while observed."

Home and Whitaker [284] presented an argument leading to the opposite conclusion. In the orthodox treatment above, they said that the crucial role of the wave-function collapse was not the change from a linear combination of eigenfunctions to a single one, but the change from pure to mixed state. They went on to claim that, although, for an ensemble interpretation, the fact that a particular result is obtained in a measurement may be of reduced importance, the fact that any result is obtained is sufficient that the state becomes mixed. The "surviving" term in the wave function is cut off from the "decayed" term, and, as shown above, this serves to reduce, or, in the limit, eliminate, the possibility of decay.

This argument in effect relies on the discussion of sections 5.4, 6.1 and 6.2 that, to represent a measurement result, even ensemble supporters must consider a mixed state following a measurement. Thus it might not be convincing to those reported in those sections who believe that a pure state may represent the result of a measurement. Similarly a distinction might be made between different versions of many-worlds interpretation. Those who explicitly talk of separation of decayed and surviving species into different worlds [292] might predict a quantum Zeno effect; those who use an unchanged superposition $[23,293]$ might claim that it is avoided.

A more recent paper [294] suggests that this argument might be misleading. It shows that, in the terms of this paper, formation of an improper mixture at a measurement, which must occur in any interpretation, is sufficient for the prediction of the quantum Zeno effect. Immediately after a measurement, it is pointed out that we should really use a combined wavefunction for measuring and measured systems, of the form $a_{\mathrm{s}} \phi_{\mathrm{s}} \psi_{\mathrm{s}}+a_{\mathrm{d}} \phi_{\mathrm{d}} \psi_{\mathrm{d}}$, where $\psi_{\mathrm{s}}$ and $\psi_{\mathrm{d}}$ are states of the measuring system. Because $\psi_{\mathrm{s}}$ and $\psi_{\mathrm{d}}$ are macroscopic, they cannot interfere, so $\phi_{\mathrm{d}}$ and $\phi_{\mathrm{s}}$ are cut off from each other, thus leading to the quantum Zeno prediction, as above.

An explicit treatment, involving the states of the measuring system, and again restricted to two levels, is as follows [295]. If the decaying system is allowed to evolve for a time $T$ uninterrupted by an 
external intervention, the final wave function of the coupled system is given by

$$
\Psi(T)=\alpha(T) \phi_{\mathrm{s}} \psi_{\mathrm{s}}+\beta(T) \phi_{\mathrm{d}} \psi_{\mathrm{d}}
$$

the survival probability being

$$
P_{s}=|\alpha(T)|^{2} \text {. }
$$

It is pointed out that no collapse of wave function is assumed. If, on the other hand, a measurement is performed at $T / 2$, and the system evolves freely between $t=0$ and $T / 2$, and between $t=T / 2$ and $T$, the coupled wave function at $T$ is given by

$$
\Psi(T)=[\alpha(T / 2)]^{2} \phi_{\mathrm{s}} \psi_{\mathrm{s}}+[\alpha(T / 2) \beta(T / 2)] \phi_{\mathrm{d}} \psi_{\mathrm{s}}-[\beta(T / 2)]^{2} \phi_{\mathrm{s}} \psi_{\mathrm{d}}+[\beta(T / 2) \alpha(T / 2)] \phi_{\mathrm{d}} \psi_{\mathrm{d}} .
$$

Because both the $\phi \mathrm{s}$, and the $\psi \mathrm{s}$, are orthogonal, a measurement at $T$ will demonstrate a survival probability given by

$$
P_{\mathrm{s}}^{\prime}=|\alpha(T / 2)|^{4} \text {. }
$$

We may easily generalise to the case of measurements at $T / n, 2 T / n, \ldots, T$, where

$$
P_{\mathrm{s}}^{\prime}=|\alpha(T / n)|^{2 n}
$$

For the case where

$$
\alpha(T)=1-k T^{2} / 2
$$

it follows that

$$
P_{\mathrm{s}}^{\prime}=1-k T^{2} / n
$$

and $P_{s}^{\prime}$ tends to 1 as $n$ tends to $\infty$, the usual Zeno result, obtained without collapse of wave function.

Thus one may say, "it is the process of measurement itself - the irreversible interaction of system and observing apparatus - that separates the wave function components". If this is accepted, the prediction of the effect becomes independent of interpretation, and a property of the theory itself. Since, as has been said, the prediction of the effect is embarrassing, we may well try to eliminate it (and suggestions are given in several of the papers cited), but they must be by closer analysis, or adjustment, of the theory itself, not by suggesting a novel interpretation.

How does that leave those ensemble interpretations that were said not to predict the effect? The argument from lack of collapse is easily seen to be incomplete. The problem of the PIV approaches seems to be more worrying. Let us first examine the idea that all atoms are programmed to decay at a particular time. Clearly that cannot be sustained, unless one acknowledges that these times are changed by the measurement (so that the altered decay profile will show the slowing of the decay caused by the measurement). So, in a similar way to that discussed at the end of section 5.1, PIVs may survive this challenge, but only by making them so measurement-dependent that their attractive features very much diminish. 
The type of PIV theory which does not assume that each decay time is preprogrammed, but that each atom has a probability of decaying at any time, must similarly accept that the probability is affected by the measurement.

\subsection{Bell's theorem}

Bell's theorem [21] relates to what has been one of the main themes of this paper - the question of whether noncommuting observables, in this case components of spin, may be regarded as simultaneously "having" values (or, more generally, hidden variables existing that can guarantee which results will be obtained if the observables are measured). It showed that any such theory which produces the usual quantum-mechanical results cannot be local. (We call this approach 1.) (Note that this is the usual view taken of Bell's work, but ignores his own argument from locality to hidden variables mentioned in section 6.1.)

Subsequently, Stapp [296] and others [297] have put much effort into eliminating the need for any assumption of hidden variables, though there has been questioning of how successful this has been by Kraus [82, p. 461] and others [298, 299]. (See also ref. [274].) (This we call approach 2.)

It would seem immediately clear that the PIV interpretation comes directly into the category of approach 1, and thus conflicts with locality. A minimal ensemble interpretation would be considered along with an orthodox interpretation; either would be immune to approach 1, but, assuming the success of approach 2, which is interpretation-independent, neither could maintain locality.

It is against this general background that we examine a pair of papers by Ballentine [300] and Stapp [301]. These resulted from Stapp's paper [88] on the Copenhagen interpretation discussed in section 3. In this paper, Stapp characterises the approaches of Popper and Ballentine as a "real-particle interpretation", "real particles" being defined as "tiny localised objects, or disturbances, or singularities, or other things that stay together as particles should, and do not spread out like waves. According to this interpretation", Stapp says, "the probability functions of quantum theory describe, typically, the probability that a real particle is in such-and-such a region". (Later [296], Stapp was to characterise Ballentine's approach as a "classical-world interpretation" embracing the quantum potential [19] and stochastic approaches.) His view of it is thus definitely as a structured rather than a minimal ensemble. Stapp says that Bell's theorem does not absolutely rule out the real-particle interpretation. (If he is, at this stage, including Bohm's work [19], that is clearly the case.) He does suggest, though, "a dynamical theory based on such a real entity would have no testable dynamical consequences".

Ballentine [300] responds that Stapp's "implied claim that Bell's theorem favors Bohr's interpretation over others is not justified". In his argument, he lists three interpretations of quantum theory: (A) absolute $\psi$ (von Neumann) (section 3.1); (B) "statistical ensemble"; and (C) hidden variables. He says that $\mathrm{C}$ undertakes to predict the results of individual measurements, while $\mathrm{B}$ insists on a realistic description of individual systems and events.

Ballentine, like ourselves above, discusses two approaches to Bell's ideas, one rigorous, the other perhaps open to question. The first (our approach 1), he says, requires locality and causality. The latter term replaces "the language of hidden variables" of Bell's initial paper. It enables one to "speak, conceptually of the value which a spin component would have exhibited if it had been measured. This would be the case if either the value of the spin component were already present before the measurement, or the particular value obtained from measurement were uniquely determined by some other variable." In our terms this definition would seem at least very close to either PIVs or deterministic hidden variables, though it does also open up the very delicate question of "counterfactual definiteness" [298, 299, 302], which lies beyond the scope of this paper. 
The second form (our approach 2), though, drops causality (or the equivalent). Ballentine attributes this view to Stapp, though he finds it plausible himself, but he insists that, if it is accepted, "one cannot invoke it to advance one interpretation over another ... If the principle of locality ... is invalid, this is not just an embarrassment for one or two interpretations of QM but rather a profound surprise for everyone not only for quantum physicists."

This is essentially a fair argument, to which Stapp [301] makes one point (and we add another). Stapp suggests effectively that a "real-particle interpretation" (or equivalent) is more vulnerable to the thrust of Bell's work than the Copenhagen interpretation, because it claims more. The EPR argument was a criticism of Copenhagen saying that it "claimed too little" (in our terms), and should be completed. But, Stapp says, "[the] EPR argument is invalidated by the extended Bell's theorem, which shows that the two assumptions of the EPR argument, namely EPR locality, and validity of the statistical predictions of quantum theory, are mutually incompatible".

On the other hand, Stapp says: "The extended Bell's theorem shows that any theory based on the real-particle interpretation, or any other attempt to picture nature as a collection of localised real parts that interact via some (deterministic or statistical) dynamic law, must have a dynamic law that violates the principle of local causality." This means that "the real or actual course of events (as opposed to the statistical behaviour of aggregates) ... on earth would depend strongly on what is happening all over the universe. Such a situation would seem to preclude any test of the theory that goes beyond a test of the statistical predictions of quantum theory itself. Hence the extended Bell's theorem lends strong support to the Copenhagen claim that quantum theory is pragmatically complete."

Our own argument goes back to Ballentine's listing of A, B and C. In our opinion, Ballentine's argument would be strong if $\mathrm{B}$ were a minimal ensemble interpretation. However, in a quite different fashion, Ballentine defines the "statistical ensemble interpretation" by saying: "A state vector describes certain statistical properties of an ensemble of similarly prepared systems. Individual systems possess definite, although perhaps unknown, values of certain fundamental observables (such as position) which are not described by the state vector unless it happens to be an eigenvector of such an observable." In other words, this is perhaps the clearest espousal of a PIV ensemble that Ballentine has ever made. As such, B is clearly a hidden-variables theory, and it is pointless to separate it from C. As such again, it is highly vulnerable even to approach 1 of Bell's work, which specifically relates to hidden variables.

We now turn to two later papers. Kunstatter and Trainor [303] (KT) examine the notion they attribute to Ballentine that "the Statistical Interpretation is in closer accord with the EPR notion of reality". However they argue that locality is "fundamentally inconsistent with quantum mechanical predictions whether one does or does not adopt the statistical viewpoint. In fact, any attempt to reinstate the validity of [locality] via a statistical argument involves an even greater conceptual sacrifice than does the conventional view."

Their argument follows the well-known analysis of Mermin [304], whose (thought-) experiment allows spin measurements in either wing of an EPR-type system along one of three equally spaced directions. Following Mermin, and so using a PIV ensemble, KT say, "the EPR notion of reality requires individual particles once separated to have elements of reality corresponding to the spin (either up or down) for each particle along each of the axes [on each wing]". They thus divide the pairs of particles into eight sets. They are able to show, however, that the quantum-mechanical predictions for a number of possible choices of experiment, are incompatible with any assignment of proportions of particle pairs to each set.

One way round the dilemma would be to allow these proportions to be affected by the switch settings, but KT describe that as "a situation at least as mysterious and bizarre as the nonlocal effects implied in 
the Copenhagen interpretation". KT conclude, "Bell's theorem forces us to live with the mysterious nature of quantum predictions. Despite its promise of easing our conceptual difficulties, the statistical interpretation, if anything, adds to them." They suggest that both EPR and a [PIV-] ensemble interpretation "founder on the rocks of considering separated particles as individual systems." Treating the two particles as a single system avoids the difficulties but "one has clearly not avoided the nonlocal aspect of the single system".

KT say, "the statistical predictions of quantum mechanics involve nonlocal correlations and this point stands independently of whether the statistics can or cannot ever be replaced by a more deterministic hidden-variables theory". They add, though, "the inherent nonlocality implied by Bell's theorem should present us with no more conceptual difficulties than we already accept (in either the Copenhagen or statistical interpretation) in quantum statistics and its connection with the spin of elementary particles". In fact, "specific difficulties are avoided if one sticks to the statistical behaviour of the composite system. These remarks apply whether we are considering the statistics in the sense of Bohr or in the sense of Ballentine (so-called statistical interpretation)."

Gillespie [305] has also essentially repackaged the Bell argument, in his case in the variation of Wigner [216], to show explicitly that it applies to PIV ensembles. He starts off by examining a quantum system with two observables, $A$ and $B$, whose corresponding operators are nondegenerate and noncommuting. He shows that it is trivial to construct a simple (PIV-)ensemble interpretation for this case, so that the four quantities, $f_{A B}\left(a_{ \pm}, b_{ \pm}\right)$, the fractions of the systems in the ensemble with $A=a_{ \pm}, B=b_{ \pm}$, will yield the quantum predictions for any measurement of $A$ or $B$. (He points out that only three of the $f_{A B}$ are defined, so there is an infinite number of ways of assigning (non-negative) numbers to the $f_{A B}$.

Gillespie now proceeds to a system $S$ composed of two identical subsystems $S_{1}$ and $S_{2}$ of the kind just considered. The state vector lies in a four-dimensional "tensor product" space. In this case, three noncommuting nondegenerate observables $A_{\mu}, B_{\mu}$, and $C_{\mu}$ are required for $S_{\mu}(\mu=1,2)$, the eigenbases for $B_{\mu}$ and $C_{\mu}$ being rotated from that of $A_{\mu}$ by $\phi$ and $2 \phi$ respectively. Thus any state vector $|\psi\rangle$ of the compound system may be represented in nine ways.

Gillespie now says: "In an ensemble interpretation, the compound state vector $|\psi\rangle$ is assumed to represent a statistical ensemble of identically prepared systems, each of which has precise values for ... $A_{1}, B_{1}, C_{1}, A_{2}, B_{2}$ and $C_{2}$." In analogy to the previous case, there should now be 64 (non-negative) variables $f\left(a_{i}, b_{j}, c_{k}, a_{l}, b_{m}, c_{n}\right)$ for the fraction of systems with given values. There are nine joint measurements that may be performed of one spin component on $S_{1}$, and one on $S_{2}$; each may give four different results, the probabilities of which may be calculated from quantum theory for any $|\psi\rangle$, so we are left with 36 equations for 64 unknowns (but, of course, all 64 must be nonnegative which makes the situation difficult to assess).

For $|\psi\rangle$ given by the Bell singlet state, there is considerable simplification. Six of the 36 measurement equations conspire with the non-negativity rule to make all but eight of the $f$ variables zero (the surviving ones being those in which $a_{i} \neq a_{1}, b_{j} \neq b_{m}, c_{k} \neq c_{n}$ ). There are now 30 remaining equations for these 8 remaining (non-negative) $f$ variables, and it is fairly easy to obtain the usual Bell's inequality, which is not, of course, obeyed by the quantum predictions. Gillespie concludes: "A simple measurement prediction ensemble cannot be constructed for this state." If one assumes locality, he remarks, "The conclusion that a physical system will not always have values for some of its observables seems almost inescapable." Alternatives, "such as allowing negative probabilities, or nonlocality, seem to betray [the] original promise [of the simple ensemble approach] of providing a 'common sense' interpretation of quantum theory." 
Recently Greenberger, Horne and Zeilinger have presented an extremely interesting development of Bell's theorem [306]. (See also refs. [307,308].) They consider a system of three mutually wellseparated and correlated spin-1/2 particles, and discuss the state

$$
|\psi\rangle=(1 / \sqrt{2})(|1,1,1\rangle-|-1,-1,-1\rangle)
$$

Using the EPR reality criterion and the locality condition, they are able to demonstrate that the components $S_{x}$ and $S_{y}$ for each of the three spins are elements of reality with pre-assigned values, and are further able to show that these values obey the equation

$$
\alpha=S_{x}^{1} S_{x}^{2} S_{x}^{3}=\hbar^{3} / 8
$$

However, using (6.8), it is a straightforward result of quantum theory that

$$
\sigma_{x}^{1} \sigma_{x}^{2} \sigma_{x}^{3}|\psi\rangle=-|\psi\rangle
$$

and so $\alpha=-\hbar^{3} / 8$, in contradiction to $(6.15)$.

This demonstrates an incompatibility between quantum theory and the conjunction of the EPR reality criterion with the locality condition. The important advance on Bell's theorem is that the correlations are perfect rather than statistical, and hence inequalities are dispensed with.

In the context of the present paper, the result can only serve to reinforce the problems of PIV interpretations. The existence of PIVs for the $S_{x}$ and $S_{y}$ components of the three particles would certainly explain the expected correlations between measurements on the various components. It seems to be contradicted, however, by (6.16). Of course, one might question the significance of (6.16). It might be argued that there should be PIVs for $S_{x}^{i}$ and $S_{v}^{1}$, since these correspond to dynamical variables, but the triple product does not correspond to a dynamical variable, and so there is no requirement for a PIV. The argument that $S_{x}^{1}, S_{x}^{2}$ and $S_{x}^{3}$ "possess" values, but $S_{x}^{1} S_{x}^{2} S_{x}^{3}$ does not do so, must seem, though, wholly artificial.

\section{Conclusions}

Any quick assessment of the capability of ensemble interpretations must depend on the scope of the term. If one uses the broadest possible definition in which "structured" ensembles, with an underlying notion of giving a realist description of quantum phenomena, are included, theories usually called hidden-variables theories $[19,122]$ or stochastic theories (section 4.6), it is reasonable to make a positive judgement. One is impressed by the variety and vitality of such approaches (though in EPR-type situations, they are, of course, subject to Bell's theorem, and hence are not compatible with quantum mechanics unless locality is permitted to be violated at the realist level of individual values).

If, on the other hand, one restricts oneself to the types of interpretation more generally termed "ensemble", PIV (premeasurement initial values for all observables) ensemble theories [1], and minimal ensemble theories [2], matters are inevitably more contentious.

Many, particularly physicists, may feel that, in even discussing the PIV idea, we are criticising an interpretation of straw. We cannot agree. The idea is extremely powerful in both the physical and more philosophical literature (sections 4.3 and 4.4, and refs. [2,11,229] among others). Even when it is not 
expressly acknowledged, or perhaps even recognised, we suspect that PIV assumptions creep into analysis to make applications of general ensemble ideas seem more powerful than they otherwise might. (We might also mention the unwillingness of some authors to realise that PIVs are just a species of hidden variable.) In addition, probably the most popular argument among physicists against the interpretation, that based on the uncertainty principle, is not satisfactory.

To say this, does not imply, of course, that there are not sound arguments against PIVs, and we have aimed to present these systematically in sections 5.1 and 5.2 (and, to an extent, in 5.5). To say the least, they include a large amount of obstacles that PIV interpretations must overcome if they are to gain respectability.

This brings us to the minimal interpretation (section 5.3). This is always allowable. It may be encouraged by adherence to a relative-frequency (rf) interpretation of probability. Jammer reports [4, p. 119] that such an interpretation may be "more palatable to the majority of physicists". While this may be the case, our discussion of probability shows that rf interpretations are not really more directly tied to experimental results than individual system interpretations; we showed that both types of interpretation have conceptual problems, and indeed many of them are analogous.

The important questions are, though: Are minimal ensemble interpretations really different from orthodox interpretations? Do they solve the problems of orthodox interpretations? Jammer [4, p. 441] appears to be dubious about there being any real difference. In the end, the crucial point is whether the pure state which an ensemble yields following a measurement constitutes a solution to the problem. The argument that it does so, being in some way equivalent to a mixture, is put in section 5.4, together with strong criticism from Sudbery [91] and Leggett [132, p. 85].

The "paradoxes" of quantum theory (section 6) are felt by many to be very good evidence for the ensemble view. Analysis of the EPR and Schrödinger's cat problems show that the argument that the (minimal) ensemble position removes any difficulties is essentially just the one mentioned; a pure state must be regarded as (in effect) a mixture. We do not find the quantum Zeno "paradox" or Bell argument distinguishes ensemble position from orthodox.

On the whole, it must be said that we do not find the argument in favour of (minimal or PIV) ensembles to be of great strength. Our position is not intended to be a "comfortable" one, encouraging bland acceptance of the orthodox status quo. Rather we see the ensemble interpretation as the "comfortable" option, creating the illusion that all difficulties may be removed by taking one simple step.

Where then should one go? There seem to be two immediate options: (1) deterministic or stochastic realist models like the hidden-variables theories; or (2) some variant of the orthodox position. To indicate that the "orthodox" side of things is not as monolithic as its opponents sometimes maintain, in section 2 we presented an examination of the various "orthodox" positions, highlighting Stapp's view [88] that von Neumann's ideas were opposed to those of Bohr, not equivalent. Even as simple a view as that of Maxwell [230,240], which may perhaps be fairly regarded as an orthodox approach, has its attractions - as well as its problems. But over and above these, we would encourage a catholicity of approach, rather than the "soporific", whether the sleep-inducing slogan be "complementarity" or "ensembles".

In the context of this paper, and to ensure even a modicum of coherence, a particular position has been generally maintained. We have endeavoured, though, especially in sections 4.5 and 5.6, but also elsewhere, to present a range of views, sometimes differing from our own, and, as much as possible, in the actual words of their authors. In this way, we hope that even those who may not accept our own stance may be provided with enough information and analysis to develop their own views. 


\section{Acknowledgements}

D.H. acknowledges support from the Department of Science and Technology of the Government of India while carrying out the first stages of this work, and a Fellowship of the Commission of the European Community during its completion. We would like to thank Dr. J.R. Fox and Dr. D.T. Gillespie for sending us unpublished manuscripts, and would also thank Dr. Gillespie for interesting discussions.

\section{References}

[1] D. Home and M.A.B. Whitaker, Phys. Lett. A 115 (1986) 81.

[2] P. Gibbins, Particles and Paradoxes (Cambridge Univ. Press, Cambridge, 1987).

[3] L.E. Ballentine, Rev. Mod. Phys. 42 (1970) 385.

[4] M. Jammer, The Philosophy of Quantum Mechanics (Wiley, New York, 1974).

[5] N. Bohr, Atomic Theory and the Description of Nature (Cambridge Univ. Press, Cambridge, 1934).

[6] W. Heisenberg, The Physical Principles of the Quantum Theory (Univ. of Chicago Press, Chicago, 1930).

[7] A. Einstein, J. Franklin Institute 221 (1936) 349.

18] A. Einstein, B. Podolsky and N. Rosen, Phys. Rev. 43 (1935) 777.

[9] J.C. Slater, J. Franklin Institute 207 (1929) 449.

[10] E.C. Kemble, J. Franklin Institute 225 (1938) 263.

[11] K. Popper, Logik der Forschung (Springer, Vienna, 1935); The Logic of Scientific Discovery (Basic Books, New York, 1959).

[12] P. Langevin, La Notion de Corpuscle et d'Atome (Hermann, Paris, 1934)

[13] H. Margenau, Phys. Rev. 49 (1936) 240.

[14] J. von Neumann, Mathematical Methods of Quantum Mechanics (Princeton Univ. Press, Princeton, 1955).

[15] D.I. Blokhintsev, Principles of Quantum Mechanics (Reidel, Dordrecht, 1964). (Russian original published in 1949.)

[16] V. Fock, Czech. J. Phys. 7 (1957) 643.

[17] D.I. Blokhintsev, The Philosophy of Quantum Mechanics (Reidel, Dordrecht, 1968).

[18] P.A. Schilpp, ed., Albert Einstein: Philosopher-Scientist (Library of the Living Philosophers, Evanston, 1949)

[19] D. Bohm, Phys. Rev. 85 (1952) 166, 180.

[20] J.S. Bell, Rev. Mod. Phys. 38 (1966) 447.

[21] J.S. Bell, Physics 1 (1964) 195.

[22] J.S. Bell, Speakable and Unspeakable in Quantum Mechanics (Cambridge Univ. Press, Cambridge, 1989).

[23] H. Everett, Rev. Mod. Phys. 29 (1957) 454.

[24] B.S. de Witt, in: Battelle Rencontres 1967 - Lectures in Mathematics and Physics, eds C. de Witt and J.A. Wheeler (Benjamin, New York, 1968) p. 318.

[25] J.B. Hartle, Am. J. Phys. 36 (1968) 704.

[26] L.N. Cooper and D. Van Vechten, Am. J. Phys. 37 (1969) 1212.

[27] E. Schrödinger, Annales de l'Institut Henri Poincaré 2 (1932) 1212.

[28] R. Fürth, Z. Phys. 81 (1932) 143.

[29] F. Bopp, Z. Naturforsch. 7a (1952) 82.

[30] P. Pearle, Am. J. Phys. 35 (1967) 742.

[31] J.L. Park, Am. J. Phys. 36 (1968) 211.

[32] L.W. Branscomb, Rev. Mod. Phys. 42 (1970) 357

[33] C.B. Chiu, E.C.G. Sudarshan and B. Misra, Phys. Rev. D 16 (1977) 520.

[34] A. Aspect, P. Grangier and G. Roger, Phys. Rev. Lett. 49 (1982) 1804.

[35] A.J. Leggett, in: The Lesson of Quantum Theory, eds J. de Boer, E. Dal and O. Ulfbeck (North-Holland, Amsterdam, 1986).

[36] H. Rauch, H. Treimer and U. Bonse, Phys. Lett. A 47 (1974) 369.

[37] A.B. Pippard, Eur. J. Phys. 7 (1986) 43.

[38] J.A. Wheeler and W.H. Zurek, eds, Quantum Theory and Measurement (Princeton Univ. Press, Princeton, 1983)

[39] L.E. Ballentine, in: Fundamental Questions in Quantum Physics, eds L.M. Roth and A. Inomata (Gordon and Breach, New York, 1986) pp $65-75$.

[40] A. Landé, Quantum Mechanics (Pitman, London, 1951).

[41] A. Landé, From Dualism to Unity in Quantum Theory (Cambridge Univ. Press, Cambridge, 1960); New Foundations of Quantum Mechanics (Cambridge Univ. Press, Cambridge, 1965). 
[42] W. Duane, Proc. Natl. Acad. Sci. 9 (1923) 158.

[43] H. Mehlberg, in: Current Issues in the Philosophy of Science (Holt, Rinehart and Winston, New York, 1961) pp. 360-370.

[44] P. Weingartner and G. Zecha, eds, Induction, Physics and Ethics (Reidel, Dordrecht, 1970).

[45] R. Weatherford, Philosophical Foundations of Probability Theory (Routledge and Kegan Paul, London, 1982).

[46] J.R. Lucas, The Concept of Probability (Clarendon, Oxford, 1970).

[47] H. Jeffreys, Theory of Probability (Clarendon, Oxford, 1948).

[48] H. Reichenbach, The Theory of Probability (University of California, Berkeley, 1949).

[49] H. Poincaré, Science and Hypothesis (Dover, New York, 1952).

[50] H.E. Kyburg and H.E. Smokler, eds, Studies in Subjective Probability (Wiley, New York, 1964).

[51] H. Poincaré, Calcul des Probabilités (Gauthier, Paris, 1912).

[52] R. von Mises, Probability, Statistics and Truth (George Allen and Unwin, London, 1957).

[53] C.I. Lewis, An Analysis of Knowledge and Valuation (Open Court, La Salle, 1962).

[54] J.M. Keynes, A Treatise on Probability (Macmillan, London, 1921).

[55] A.N. Kolmogorov, Foundations of the Theory of Probability (Chelsea, New York, 1956).

[56] T.L. Fine, Theories of Probability (Academic Press, New York, 1973).

[57] P.S. Marquis de Laplace, A Philosophical Essay on Probabilities (Dover Publ., New York, 1951).

[58] I. Todhunter, A History of the Mathematical Theory of Probability From the Time of Pascal to that of Laplace (Chelsea, New York, 1949).

[59] R. Carnap, Logical Foundations of Probability (Routledge and Kegan Paul, London, 1950).

[60] S.E. Toulmin, The Uses of Argument (Cambridge Univ. Press, Cambridge, 1958).

[61] A.J. Ayer, Probability and Evidence (Columbia Univ. Press, New York, 1972).

[62] S. Körner, ed., Observation and Interpretation in the Philosophy of Physics (Dover Publ., New York, 1957).

[63] B. de Finetti, Theory of Probability (Wiley, London, 1974).

[64] L.J. Savage, The Foundations of Statistics (Wiley, New York, 1954).

[65] M. Bunge, ed., Quantum Theory and Reality (Springer, Berlin, 1967).

[66] D.H. Mellor, The Matter of Chance Cambridge Univ. Press, Cambridge, 1971).

[67] B.C. von Frassen, The Scientific Image (Clarendon, Oxford, 1980).

[68] J. Venn, The Logic of Chance (Chelsea, New York, 1963).

[69] F.J. Belinfante, Measurements and Time Reversal in Objective Quantum Theory (Pergamon, Oxford, 1975).

[70] W. Kneale, Probability and Induction (Clarendon, Oxford, 1949).

[71] J. Ville, in: Monographies des Probabilités, ed. E. Borel (Gauthier, Paris, 1939).

[72] M. Strauss, Found. Phys. 3 (1973) 265.

[73] L. Wittgenstein, Philosophical Investigations (Macmillan, New York, 1953).

[74] B. d'Espagnat, Conceptual Foundations of Quantum Mechanics (Benjamin, Reading, 1979).

[75] D. Murdoch, Niels Bohr's Philosophy of Physics (Cambridge Univ. Press, Cambridge, 1987).

[76] E. Schrödinger, Ann. Phys. 81 (1926) 109.

[77] J. Dorling, in: Schrödinger: Centenary Celebration of a Polymath, ed. C. Kilmister (Cambridge Univ. Press, Cambridge, 1987) pp. 16-40.

[78] N. Bohr, Nature 121 (1928) 580.

[79] H.J. Folse, The Philosophy of Niels Bohr (North-Holland, Amsterdam, 1985).

[80] W. Heisenberg, The Physical Principles of the Quantum Theory (Chicago Univ. Press, Chicago, 1930).

[81] W. Heisenberg, Physics and Philosophy (George Allen and Unwin, London, 1958).

[82] P. Lahti and P. Mittelstaedt, eds, Symposium on the Foundations of Modern Physics-50 Years of the Einstein-Podolsky-Rosen Gedankenexperiment (World Scientific, Singapore, 1985).

[83] M. Born, Z. Phys. 37 (1926) 863.

[84] M. Beller, Stud. Hist. Phil. Sci. 21 (1990) 563.

[85] M. Born, Z. Phys. 38 (1926) 803; Z. Phys. 40 (1927) 167.

[86] W. Pauli, Z. Phys. 43 (1927) 81.

[87] P.K. Feyerabend, in: Frontiers of Science and Philosophy, ed. R.G. Colodny (George Allen and Unwin, London, 1964) pp. 189-283.

[88] H.P. Stapp, Am. J. Phys. 40 (1972) 1098.

[89] L.E. Ballentine, Am. J. Phys. 55 (1987) 785.

[90] P. Teller, in: Proc. 1980 Biennial Meeting of the Philosophy of Science Association, vol. 2, eds P.D. Asquith and R.N. Giere (Philosophy of Science Association, East Lansing, Michigan, 1981) pp. 201-223.

[91] A. Sudbery, in: Quantum Concepts in Space and Time, eds R. Penrose and C.J. Isham (Clarendon, Oxford, 1986) pp. 65-83.

[92] P.A.M. Dirac, The Principles of Quantum Mechanics (Clarendon, Oxford, 1930).

[93] Electrons et Photons - Rapports et Discussions du Cinquieme Conseil de Physique tenu a Bruxelles 1927 sous les Auspices de l'Institut International de Physique Solvay (Gauthier-Villars, Paris, 1928).

[94] J.S. Bell in: Sixty-Two Years of Uncertainty, ed. A.I. Miller (Plenum, New York, 1990) p. 17.

[95] G. Lüders, Ann. Phys. 8 (1951) 322.

[96] L.D. Landau and R. Peierls, Z. Phys. 69 (1931) 56. 
[97] W. Pauli, in: Handbuch der Physik, eds H. Geiger and K. Scheel, vol. 24, 2nd Ed. (Springer, Berlin, 1933) pp. 83-272.

[98] L.D. Landau and E.M. Lifshitz, Quantum Mechanics, 2nd Ed. (Pergamon, Oxford. 1965).

[99] H. Margenau, Ann. Phys. (NY) 23 (1963) 469.

[100] J.L. Park and H. Margenau. Inter. J. Th. Phys. 1 (1968) 240.

[101] J.S. Bell, Found. Phys. 12 (1982) 989.

[102] N. Bohr, Naturwiss. 17 (1929) 483.

[103] L. Landau, Z. Phys. 45 (1927) 430.

[104] E.P. Wigner, in: The Scientist Speculates - An Anthology of Partly-Baked Ideas, ed. I.J. Good (Heinemann, London, 1961) pp. 284-302.

[105] R.H. Dicke and J.P. Wittke. Introduction to Quantum Mechanics (Addison-Wesiey, Reading, Mass., 1960).

[106] L.I. Schiff, Quantum Mechanics (McGraw-Hill, New York, 1949).

[107] H. Kragh, Dirac: A Scientific Biography (Cambridge Univ. Press, Cambridge, 1990).

[108] W. Moore, Schrödinger: Life and Thought (Cambridge Univ. Press, Cambridge. 1989).

[109] N. Bohr. Atomic Physics and Human Knowledge (Wiley New York. 1958).

[110] N. Bohr, Phys. Rev. 48 (1935) 697.

[111] N.R. Hanson. Am. J. Phys. 27 (1959) 1.

[112] P.A.M. Dirac, Proc. R. Soc. London A 117 (1928) 610.

[113] L. de Broglie, J. Physique Radium 8 (1927) 225.

[114] J.-P. Vigier, C. Dewdney, P.R. Holland and A. Kyprianidis, in: Quantum Implications, eds, B.J. Hiley and F.D. Peat (Routledge and Kegan Paul, London, 1987) pp. 169-204.

[115] E. Squires, The Mystery of the Quantum Worid (Adam Hilger, Bristol, 1986).

[116] E. Squires, Conscious Mind in the Physical World (Adam Hilger, Bristol, 1990).

[117] M. Gell-Mann, The Nature of the Physical World (Wiley, New York, 1979).

[118] N. Bohr, Essays 1958-1962 on Atomic Physics and Human Knowledge (Interscience. New York, 1963).

[119] F. Halzen and A.D. Martin, Quarks and Leptons (Wiley, New York, 1984).

[120] R.G. Newton. Am. J. Phys. 48 (1980) 1029.

[121] L. de la Peña and A.M. Cetto, Found. Phys. 5 (1975) 355

[122] J. Belinfante, A Survey of Hidden-Variable Theories (Pergamon, Oxford, 1973).

[123] S.P. Gudder, Stochastic Methods in Quantum Mechanics (North-Holland. New York, 1979).

[124] A. Fine, Found. Phys. 20 (1990) 967.

[125] A. Fine, The Shaky Game: Einstein, Realism and the Quantum Theory (Chicago Univ. Press, Chicago, 1986).

[126] R. Guy and R. Deltete, Found. Phys. 20 (1990) 943.

[127] B. D’Espagnat, Nuovo Cimento 21B (1974) 233.

[128] H.R. Tschudi, Helv. Phys. Acta 60 (1987) 363.

[129] L.E. Ballentine, Phys. Rev. Lett. 48 (1982) 522.

[130] P.F. Zweifel, Int. J. Th. Phys. 10 (1974) 67.

[131] C. Roychoudhuri, Found. Phys. 8 (1978) 845.

[132] Quantum Implications, eds B.J. Hiley and D. Peat (Routledge and Kegan Paul, London, 1987).

[133] M. Born, The Born-Einstein Letters (Macmillan, London, 1971).

[134] G. Hemion, Int. J. Th. Phys. 28 (1989) 1371.

[135] L.E. Ballentine, Am. J. Phys. 40 (1972) 1763.

[136] A. Fine, in: PSA: 1980, vol. 2, eds P. Asquith and R. Giere (Philosophy of Science Association, East Lansing, Michigan, 1981) pp. 535-562.

[137] A. Fine, J. Phil. 79 (1982) 733.

[138] A. Fine, Found. Phys. 19 (1989) 453.

[139] P. Pearle, Phys. Rev. D 2 (1970) 1418.

[140] J.F. Clauser and M.A. Horne, Phys. Rev. D 10 (1974) 526.

[141] F. Selleri, in: Symposium on the Foundations of Modern Physics-50 Years of the Einstein-Podolsky-Rosen Gedankenexperiment, eds P. Lahti and P. Mittelstaedt (World Scientific Singapore. 1985) p. 591.

[142] E. Santos, Physics Lett. A 139 (1989) 431.

[143] T.W. Marshall, Phys. Lett. A 100 (1984) 225.

[144] D. Home and T.W. Marshall, Phys. Lett. A 113 (1985) 183.

[145] A. Einstein, Science 91 (1940) 487.

[146] D. Bohm, Quantum Theory (Prentice-Hall, Englewood Cliffs, N.J., 1951).

[147] D. Home and S. Sengupta, Am. J. Phys. 51 (1983) 567.

[148] J.L. Martin, Basic Quantum Mechanics (Clarendon, Oxford, 1981) p. 44.

[149] E.P. Wigner, Phys. Rev. 40 (1932) 749.

[150] L.E. Ballentine, Int. J. Theor. Phys. 27 (1988) 211.

[151] R.H. Young, Found. Phys. 10 (1980) 33.

[152] V. Buonomano, Nuovo Cimento 57B (1980) 146. 
[153] V. Buonomano, Ann. Inst. Henri Poincaré 29 (1978) 379.

[154] D. Shale, Adv. Math. 32 (1979) 175.

[155] R.D. Prosser, Int. J. Theor. Phys. 15 (1976) 169.

[156] R.D. Prosser, Int. J. Theor. Phys. 15 (1976) 181.

[157] V.K. Thankappan and P.G. Hambo, Found. Phys. 10 (1980) 217.

[158] R.P. Feynman and A.R. Hibbs, Quantum Mechanics and Path Integrals (McGraw-Hill, New York, 1965).

[159] A. Das, J. Math. Phys. 21 (1980) 1513.

[160] H. Yukawa, Phys. Rev. 76 (1949) 300.

[161] E.G.P. Rowe, J. Phys. A 20 (1987) 1419.

[162] A. Fine, Phys. Rev. Lett. 48 (1982) 291.

[163] M.J.W. Hall, Found. Phys. 19 (1989) 189.

[164] R. Cox, The Algebra of Probable Inference (Johns Hopkins Univ. Press, Baltimore, 1961).

[165] L.E. Ballentine, Am. J. Phys. 54 (1986) 883.

[166] L. Cohen, Found. Phys. 18 (1988) 983.

[167] H. Ioannidou, Int. J. Theor. Phys. 22 (1982) 1129.

[168] R.I. Sutherland, J. Math. Phys. 23 (1982) 2389.

[169] H. Margenau and R.N. Hill, Prog. Theor. Phys. 26 (1961) 722.

[170] R.I. Sutherland, J. Math. Phys. 24 (1983) 1451.

[171] M.D. Srinivas, J. Math. Phys. 19 (1978) 1705.

[172] R.E. Collins, Found. Phys. 7 (1977) 475.

[173] S. Gudder, J. Math. Phys. 25 (1984) 2397; Quantum Probability (Academic Press, Orlando, Florida, 1988).

[174] I. Pitowsky, Phys. Rev. Lett. 48 (1982) 1299; Phys. Rev. D 27 (1983) 2316.

[175] S. Gudder, Int. J. Theor. Phys. 27 (1988) 193.

[176] I. Pitowsky, J. Math. Phys. 27 (1986) 1556.

[177] P. Gibbins, Phil. Sci. 48 (1981) 122.

[178] M. Jammer, Phil. Sci. 49 (1982) 478.

[179] P. Busch and P. Lahti, Philos. Sci. 52 (1985) 64.

[180] J.L. Park and W. Band, Found. Phys. 6 (1976) 157.

[181] G. Ludwig, Commun. Math. Phys. 4 (1967) 331; 9 (1968) 1.

[182] E.B. Davies, Quantum Theory of Open Systems (Academic Press, New York, 1976).

[183] E.B. Davies and J.T. Lewis, Commun. Math. Phys. 17 (1970) 239.

[184] L. de la Peña and A.M. Cetto, Found. Phys. 12 (1982) 1017.

[185] S.M. Moore and J.A. Ramirez, Nuovo Cimento 64B (1981) 275.

[186] I. Fenyés, Z. Phys. 132 (1952) 81.

[187] E. Nelson, Dynamical Theories of Brownian Motion (Princeton Univ. Press, Princeton, 1967).

[188] L. de la Peña-Auerbach and A.M. Cetto, Int. J. Quant. Chem. 12 (1977) 23.

[189] L. de la Peña and A.M. Cetto, Rev. Mex. Fís. 18 (1969) 323.

[190] L. de la Peña, J. Math. Phys. 12 (1971) 453.

[191] J.P. Vigier and N. Cufaro Petroni, Phys. Lett. A 81 (1981) 12.

[192] L. de la Peña and A.M. Cetto, Phys. Rev. D 3 (1971) 795.

[193] D.L. Weaver, Phys. Rev. Lett. 40 (1978) 1473.

[194] N.S. Kalitsin, Sov. Phys. - JETP 25 (1953) 407.

[195] E. Adirovich and M. Podgorietski, Sov. Phys. - JETP 26 (1954) 150.

[196] P. Braffort, M. Spighel and C. Tzara, C.R. Acad. Sci. 239 (1954) 157.

[197] A.A. Sokolov and V. Tumanov, Sov. Phys. - JETP 3 (1957) 958.

[198] T.W. Marshall, Proc. R. Soc. A 276 (1963) 475; Proc. Cambridge Philos. Soc. 61 (1965) 537.

[199] T.H. Boyer, Phys. Rev. D 11 (1975) 809; in: Foundations of Radiation Theory and Quantum Electrodynamics, ed. A.O. Barut (Plenum, New York, 1980).

[200] P. Braffort, M. Surdin and A. Taroni, C.R. Acad. Sci. 261 (1965) 4339.

[201] E. Santos, Nuovo Cimento 19B (1974) 57; J. Math. Phys. 15 (1974) 1954; in: Open Questions in Quantum Physics, Invited Papers on the Foundations of Microphysics, eds G. Tarozzi and A. van der Merwe (Reidel, Dordrecht, 1985) p. 283.

[202] P. Claverie and S. Diner, C.R. Acad. Sci. 280 (1975) 1; Int. J. Quantum Chem. 12 (1977) 42.

[203] L. de la Peña and A.M. Cetto, J. Math. Phys. 2 (1979) 469; A.M. Cetto and L. de la Peña, Found. Phys. 19 (1989) 419; Phys. Rev. A 37 (1988) 1597.

[204] J.L. Jiménez, L. de la Peña and T.A. Brody, Am. J. Phys. 48 (1980) 840.

[205] P. Claverie, L. Pesquara and F. Soto, Phys. Lett. A 80 (1980) 113.

[206] E. Santamato, Lett. Nuovo Cimento 34 (1982) 493.

[207] E. Santamato, J. Math. Phys. 25 (1984) 2477. 
[208] D. Hestenes, Am. J. Phys. 47 (1979) 399.

[209] D. Hestenes, J. Math. Phys. 14 (1973) 893.

[210] S.M. Moore, Nuovo Cimento 69 B (1982) 23.

[211] E. Progovečki, Found. Phys. 14 (1984) 1147.

[212] E. Schrödinger, Naturwissenschaften 23 (1935) 807, 824, 844; translation by J.D. Trimmer, Proc. Am. Philos. Soc. 124 (1980) 323 (to which page references given).

[213] N. Bohr, H.A. Kramers and J.C. Slater, Philos. Mag. 47 (1924) 785.

[214] W. Bothe and H. Geiger, Z. Phys. 36 (1926) 177.

[215] A.H. Compton and A.W. Simon, Phys. Rev. 25 (1925) 306.

[216] E.P. Wigner, Am. J. Phys. 38 (1970) 1005.

[217] E.P. Wigner, Am. J. Phys. 39 (1971) 1097.

[218] J.F. Clauser, Am. J. Phys. 39 (1971) 1095, 1098.

[219] J.M. Jauch and C. Piron, Helv. Phys. Acta 36 (1963) 827; Rev. Mod. Phys. 40 (1966) 228.

[220] A.M. Gleason, J. Math. Mech. 6 (1957) 885.

[221] S. Kochen and E.P. Specker, J. Math. Mech. 17 (1967) 59.

[222] A. Bach, J. Math. Phys. 21 (1980) 789; Lett. Nuovo Cimento 35 (1982) 377.

[223] D. Home and S. Sengupta, Phys. Lett. A 102 (1984) 159.

[224] P.T. Lansberg and D. Home, Am. J. Phys. 55 (1987) 226.

[225] W. Feller, Probability Theory and its Applications, vol. II (Wiley, New York, 1966) p. 487.

[226] D.T. Gillespie, Am. J. Phys. 51 (1983) 520.

[227] M.O. Scully, R. Shea and J.D. McCullen. Phys. Rep. 43 (1978) 485.

[228] A. Daneri, A. Loinger and G.M. Prosperi, Nucl. Phys. 33 (1962) 297.

[229] J.M. Jauch, E.P. Wigner and M.M. Yanase, Nuovo Cimento 48B (1967) 144.

[230] N. Maxwell, Found. Phys. 6 (1976) 661.

[231] O. Donati, G.F. Missiroli and G. Pozzi, Am. J. Phys. 41 (1973) 639.

[232] P.G. Merli, G.F. Missiroli and G. Pozzi, Am. J. Phys. 44 (1976) 306.

[233] R. Rosa, Lett. Nuovo Cimento 24 (1979) 549.

[234] J. Rayski, Found. Phys. 7 (1977) 151.

[235] J. Rayski, Found. Phys. 9 (1979) 217.

[236] D. Bohm and J. Bub, Rev. Mod. Phys. 38 (1966) 453.

[237] A. Peres, Am. J. Phys. 42 (1974) 886.

[238] A. Peres, Am. J. Phys. 52 (1984) 644.

[239] A. Peres, Am. J. Phys. 54 (1986) 688.

[240] N. Maxwell, Found. Phys. 6 (1976) 275

[241] F. Selleri and G. Tarozzi, Riv. Nuovo Cimento 4 (1981) 1.

[242] H. Rauch, N. Treimer and U. Bonse, Phys. Lett. A 47 (1974) 369.

[243] K.K. Wan, Can. J. Phys. 58 (1980) 976.

[244] J. Bub, Found. Phys. 18 (1988) 701.

[245] M. Pavičić, in: Conf. Problems in Quantum Physics II; Gdansk '89, ed. J. Mizerski (World Scientific, Singapore, 1990) p. 440.

[246] P. Ghose, D. Home and A. Qadir, A Dynamical Variant of the Double-Slit Experiment, preprint, S.N. Bose Centre (Calcutta, 1991).

[247] A. Tonomura, H. Umezaki, T. Matsuda, N. Osakabe, J. Endo, and Y. Sugita, Am. J. Phys. 57 (1989) 117.

[248] H. Rauch, in: Proc. Second Inter. Symp. on the Foundations of Quantum Mechanics, eds M. Namiki, Y. Ohnuki, Y. Murayama and S. Nomura (Phys. Soc. of Japan, Tokyo, 1987) p. 16.

[249] E.E. Fitchard, Found. Phys. 9 (1979) 525.

[250] J. Scheer and M. Schmidt, Phys. Lett. A 115 (1986) 322.

[251] D.R. Mattys and E.T. Jaynes, J. Opt. Soc. Am. 70 (1980) 263.

[252] S.G. Brush, Social Studies of Science 10 (1980) 393.

[253] C.K. Jørgensen, Theor. Chim. Acta 34 (1974) 189.

[254] A. Denis, J. Langlet and J.-P. Malrieu, Theor. Chim. Acta 38 (1975) 49.

[255] P. Claverie and S. Diner, Isr. J. Chem. 19 (1980) 54.

[256] J.G.R. Tostes, Theor. Chim. Acta 59 (1981) 229.

[257] G.W. Mackey, Mathematical Foundations of Quantum Mechanics (Benjamin, New York, 1963).

[258] C. Piron, Helv. Phys. Acta 37 (1964) 439.

[259] J.M. Jauch, Foundations of Quantum Mechanics (Addison-Wesley, Reading, 1968).

[260] P. Mittelstaedt, Quantum Logic (Reidel, Dordrecht, 1978).

[261] G. Ludwig, An Axiomatic Basis for Quantum Mechanics I (Springer, Berlin, 1985).

[262] P.J. Lahti, Int. J. Theor. Phys. 19 (1980) 789.

[263] P. Busch and P.J. Lahti, Phys. Rev. D 29 (1984) 1634. 
[264] W.M. de Muynck, P.A.E.M. Janssen and A. Santman, Found. Phys. 9 (1979) 71.

[265] H. Martens and W.M. de Muynck, Found. Phys. 20 (1990) 255, 357.

[266] P. Suppes, Philos. Sci. 22 (1961) 378.

[267] J.G. Taylor and E. Balanovski, Nature 279 (1979) 631.

[268] A.A. Ross-Bonney, Nuovo Cimento 30B (1975) 55.

[269] J.R. Fox, Am. J. Phys. 51 (1983) 49.

[270] C.D. Cantrell and M.O. Scully, Phys. Rep. 43 (1978) 499.

[271] M.A.B. Whitaker and I. Singh, Phys. Lett. A 87 (1981) 9.

[272] M.A.B. Whitaker and I. Singh, J. Phys. A 15 (1982) 2277.

[273] J.S. Bell, J. Physique, Colloque C2, 42 (1981) p. C2 41 .

[274] M.A.B. Whitaker and J.G. Dennison, Phys. Lett. A 133 (1988) 466.

[275] E. Breitenberger, Nucl. Phys. A 211 (1973) 623.

[276] J.G. Loeser, Am. J. Phys. 52 (1984) 1089.

[277] L.E. Ballentine, Am. J. Phys. 54 (1986) 947.

[278] J.G. Loeser, Am. J. Phys. 54 (1986) 948.

[279] C.B. Chiu, E.C.G. Sudarshan and B. Misra, Phys. Rev. D 16 (1977) 520.

[280] B. Misra and E.C.G. Sudarshan, J. Math. Phys. 18 (1977) 765.

[281] A. Peres, Am. J. Phys. 48 (1980) 931.

[282] I. Singh and M.A.B. Whitaker, Am. J. Phys. 50 (1982) 882.

[283] E. Joos, Phys. Rev. D 29 (1984) 1626.

[284] D. Home and M.A.B. Whitaker, J. Phys. A 19 (1986) 1847.

[285] W.M. Itano, D.J. Heinzen, J.J. Bollinger and D.J. Wineland, Phys. Rev. A 41 (1990) 2295.

[286] A. Peres, Ann. Phys. (NY) 129 (1980) 33.

[287] A.M. Lane, Phys. Lett. A 104 (1984) 91.

[288] P.T. Greenland and A.M. Lane, Phys. Lett. A 134 (1989) 429.

[289] F. Aumayr, J. Hung and S. Suckewer, Phys. Rev. Lett. 63 (1989) 1215.

[290] A. Peres and A. Ron, Phys. Rev. A 42 (1990) 5720.

[291] L.E. Ballentine, Found. Phys. 20 (1990) 1329.

[292] B.S. de Witt, Phys. Today 23, part 9 (1970) 30.

[293] E.J. Squires, Eur. J. Phys. 8 (1987) 171.

[294] M.A.B. Whitaker, Eur. J. Phys. 10 (1989) 71.

[295] D. Home and M.A.B. Whitaker, A Critical Re-examination of the Quantum Zeno Paradox, submitted to J. Phys. A (1991).

[296] H.P. Stapp, Am. J. Phys. 53 (1985) 306.

[297] P.H. Eberhard, Nuovo Cimento B 46 (1978) 392.

[298] B. d'Espagnat, Phys. Rep. 110 (1984) 201.

[299] R.K. Clifton, J.N. Butterfield and M.L.G. Redhead, Br. J. Philos. Sci. 41 (1990) 5.

[300] L.E. Ballentine, Am. J. Phys. 42 (1973) 81.

[301] H.P. Stapp, Am. J. Phys. 42 (1973) 84.

[302] H.P. Stapp, Found. Phys. 18 (1988) 427, 833.

[303] G. Kunstatter and L.E.H. Trainor, Am. J. Phys. 52 (1984) 598.

[304] N.D. Mermin, Am. J. Phys. 49 (1981) 490.

[305] D.T. Gillespie, Am. J. Phys. 54 (1986) 889.

[306] D.M. Greenberger, M.A. Horne and A. Zeilinger, in: Bell's Theorem, Quantum Theory and Conceptions of the Universe, ed. M. Kafatos (Kluwer, Dordrecht, 1989) p. 73.

[307] D.M. Greenberger, M.A. Horne, A. Shimony and A. Zeilinger, Am. J. Phys. 58 (1990) 1131.

[308] N.D. Mermin, Am. J. Phys. 58 (1990) 731. 